Prepared for the U.S. Department of Energy

under Contract DE-AC05-76RL01830

\title{
NV Energy Large-Scale Photovoltaic Integration Study: Intra-Hour Dispatch and AGC Simulation
}
S Lu
$X$ Guo
PV Etingov
C Jin
D Meng
N Samaan

January 2013

Pacific Northwest

NATIONAL LABORATORY

Proudly Operated by Battelle Since 1965 



\title{
DISCLAIMER
}

This report was prepared as an account of work sponsored by an agency of the United States Government. Neither the United States Government nor any agency thereof, nor Battelle Memorial Institute, nor any of their employees, makes any warranty, express or implied, or assumes any legal liability or responsibility for the accuracy, completeness, or usefulness of any information, apparatus, product, or process disclosed, or represents that its use would not infringe privately owned rights. Reference herein to any specific commercial product, process, or service by trade name, trademark, manufacturer, or otherwise does not necessarily constitute or imply its endorsement, recommendation, or favoring by the United States Government or any agency thereof, or Battelle Memorial Institute. The views and opinions of authors expressed herein do not necessarily state or reflect those of the United States Government or any agency thereof.

\author{
PACIFIC NORTHWEST NATIONAL LABORATORY \\ operated by \\ BATTELLE \\ for the \\ UNITED STATES DEPARTMENT OF ENERGY \\ under Contract DE-AC0 5-76RL01830 \\ Printed in the United States of America \\ Available to DOE and DOE contractors from the \\ Office of Scientific and Technical Information, \\ P.O. Box 62, Oak Ridge, TN 37831-0062; \\ ph: (865) 576-8401 \\ fax: (865) 576-5728 \\ email: reports@adonis.osti.gov \\ Available to the public from the National Technical Information Service, \\ U.S. Department of Commerce, 5285 Port Royal Rd., Springfield, VA 22161 \\ ph: (800) 553-6847 \\ fax: $(703) 605-6900$ \\ email: orders@ntis.fedworld.gov \\ online ordering: http://www.ntis.gov/ordering.htm
}

This document was printed on recycled paper. 



\title{
NV Energy Large-Scale Photovoltaic Integration Study: Intra-Hour Dispatch and AGC Simulation
}

\author{
$\mathrm{S}$ Lu (Principal Investigator) \\ PV Etingov \\ D Meng \\ $\mathrm{X}$ Guo \\ $\mathrm{C}$ Jin \\ N Samaan
}

January 2013

Prepared for

the U.S. Department of Energy

under Contract DE-AC05-76RL01830

Pacific Northwest National Laboratory

Richland, Washington 99352 



\section{Acknowledgments}

The project team would like to thank the NV Energy colleagues, Vladimir Chadliev, Rich Salgo, Roger Halbakken, Rahn Sorensen, Barbara Allen, Steve Parks Sr., and Sophia Liang, for participating in discussions; providing generation, automatic generation control (AGC) system, scheduling process, and other valuable information; and giving helpful suggestions to improve the intra-hour dispatch and AGC simulator development. Abed Halimah at Open Systems International provided AGC system design information. The team also is grateful to Dr. Yan Liu at Pacific Northwest National Laboratory (PNNL), who helped with the software architecture design of the simulator.

The steady support and guidance from PNNL management, in particular Landis Kannberg, was critical to the completion of this research effort.

The U.S. Department of Energy Office of Electricity Delivery and Energy Reliability sponsored this project. 


\begin{abstract}
The uncertainty and variability associated with photovoltaic (PV) generation make it very challenging to balance power system generation and load, especially for high penetration cases. Higher reserve requirements and more cycling of conventional generators are generally anticipated for large-scale PV integration. However, whether the existing generation fleet is flexible enough to handle the variations and how well the system can maintain its control performance are difficult to predict. The goal of this project is to develop a software program that can perform intra-hour dispatch and automatic generation control (AGC) simulation, by which the balancing operations of a system can be simulated to answer the questions posed above. The simulator, named Electric System Intra-Hour Operation Simulator (ESIOS), uses the NV Energy southern system as a study case, and models the system's generator configurations, AGC functions, and operator actions used to balance system generation and load. Actual dispatch of AGC generators and control performance under various PV penetration levels were assessed by running ESIOS. The results could provide system operators with more insights on what to expect with different amount of PV generation in the system. This report describes the design of the simulator and presents the study results.
\end{abstract}




\section{Acronyms and Abbreviations}

$\begin{array}{ll}\text { ACE } & \text { area control error } \\ \text { AGC } & \text { automatic generation control } \\ \text { BA } & \text { balancing authority } \\ \text { CC } & \text { combined cycle } \\ \text { CPS } & \text { control performance standards } \\ \text { CT } & \text { combustion turbine } \\ \text { DB } & \text { duct burners } \\ \text { DR } & \text { demand response } \\ \text { ESIOS } & \text { Electric System Intra-Hour Operation Simulator } \\ \text { ISO } & \text { Independent System Operator } \\ \text { NERC } & \text { North American Electric Reliability Corporation } \\ \text { PNNL } & \text { Pacific Northwest National Laboratory } \\ \text { PV } & \text { photovoltaic } \\ \text { RegDn } & \text { regulation down } \\ \text { RegUp } & \text { regulation up } \\ \text { RPS } & \text { renewable portfolio standard } \\ \text { RT } & \text { real-time dispatch } \\ \text { Variable renewable generation }\end{array}$





\section{Contents}

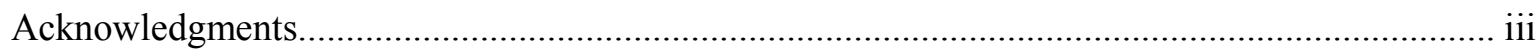

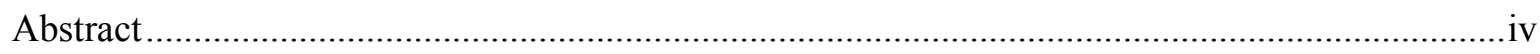

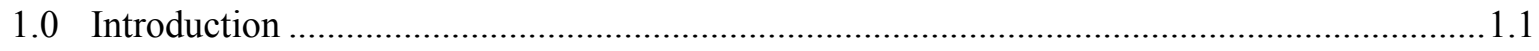

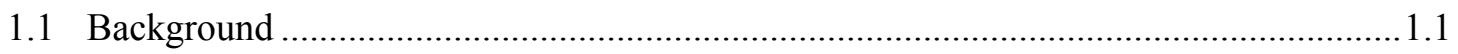

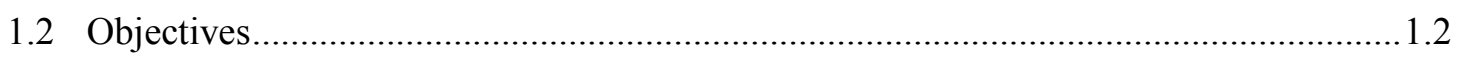

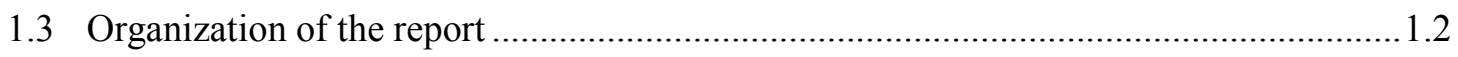

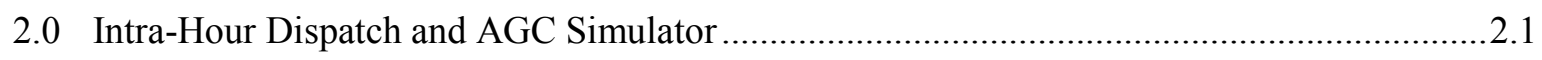

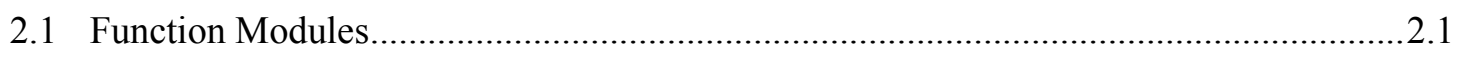

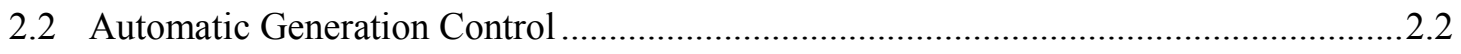

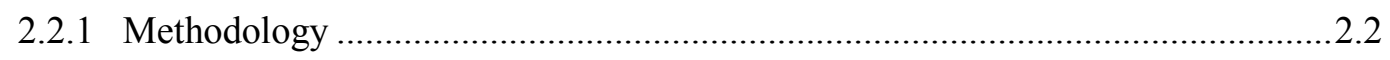

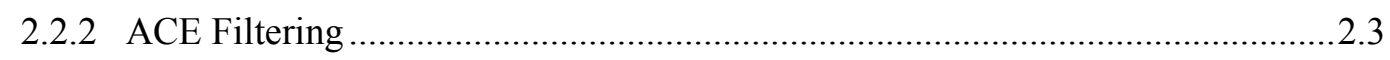

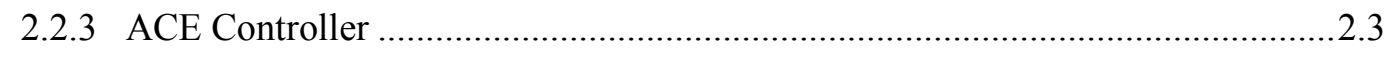

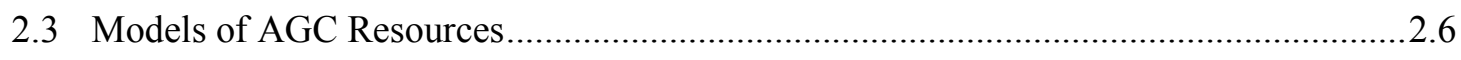

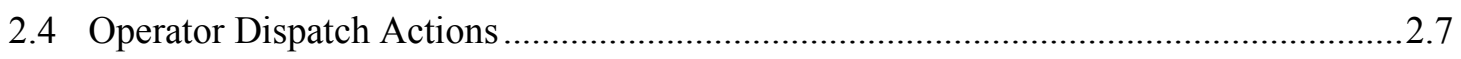

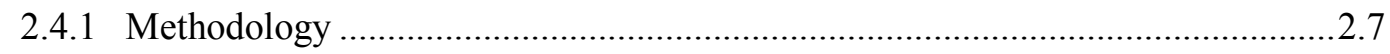

2.4.2 Startup and Shutdown of Peaking Units.....................................................2. 2.8

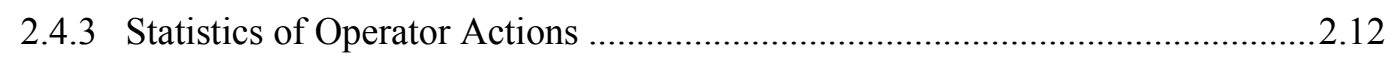

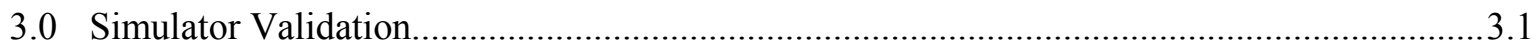

3.1 Simulation of NV Energy Southern System AGC .................................................... 3.1

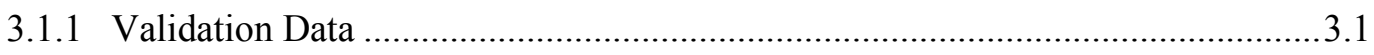

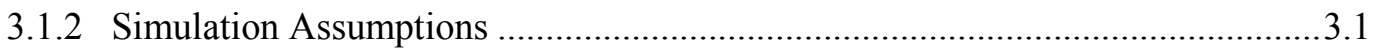

3.1.3 Validation Approaches .............................................................................. 3.1

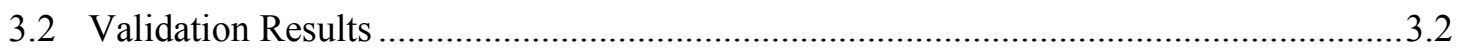

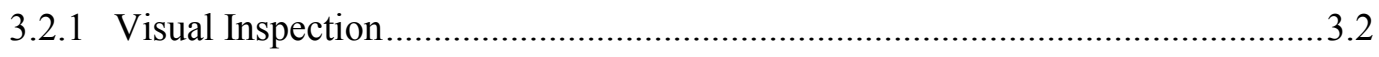

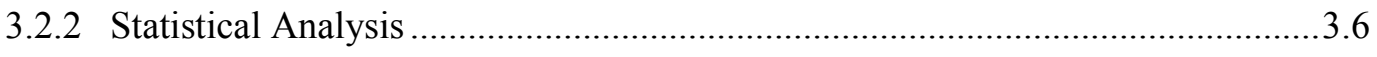

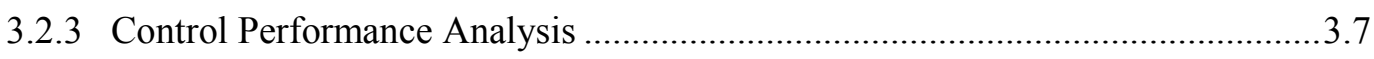

3.2.4 Results Summary of Simulator Validation....................................................... 3.7

4.0 Simulation of PV Integration Cases ......................................................................... 4.1

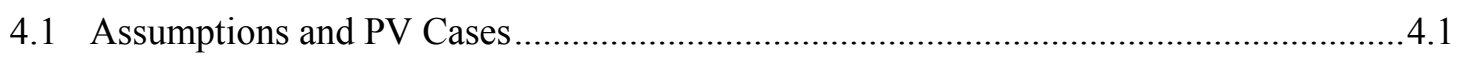

4.2 Example Results of January in Case 1A and Case 5A .................................................2

4.2.1 AGC and Peaking Units Dispatch in Case 1A .................................................4.2

4.2.2 AGC and Peaking Units Dispatch in Case 5A .................................................4.10

4.2.3 Reserve Deployment - January Case 1A vs. Case 5A .......................................4.14

4.2.4 Control Performance - January Case 1A vs. Case 5A ......................................4.19

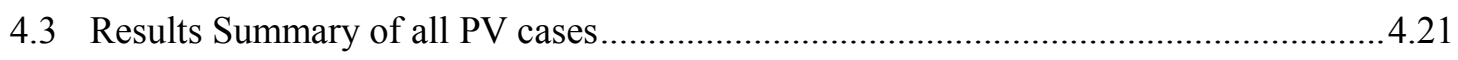

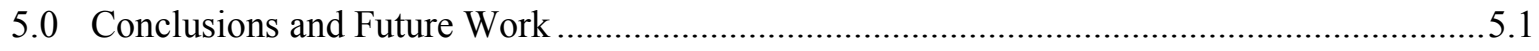




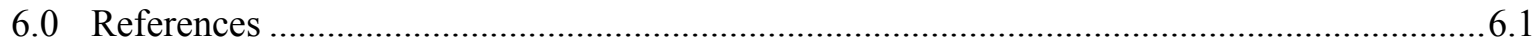

Appendix A - AGC Units Characteristics ......................................................................... A.1

Appendix B - Summary of Simulation Results of Case 1A to Case 5A ...................................... B.1 


\section{Figures}

$2.1 \quad$ Block Diagram of the Dispatch Simulator Module ................................................................ 2.1

2.2 Diagram for Real-Time Dispatch Simulation ................................................................... 2.3

2.3 Diagram for AGC Simulation ................................................................................... 2.3

2.4 ACE Distribution and Time Series with REGDB $=12 \mathrm{MW}$ and $\mathrm{EADB}=25 \mathrm{MW} \ldots \ldots \ldots \ldots \ldots . . . . . . . .2 .4$

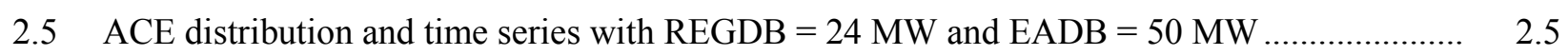

2.6 AGC unit movement and ACE on March 14 with REGDB $=12 \mathrm{MW}$................................... 2.5

2.7 AGC unit movement and ACE on March 14 with REGDB $=0$ MW ..................................... 2.6

2.8 Peaking Unit Operation Approach .............................................................................. 2.11

2.9 Startups and Runtime of Peaking Units in a Low and a High PV Penetration Case.................. 2.11

3.1 AGC Units Total Dispatch (August 1, 2012) .................................................................... 3.2

3.2 AGC Units Dispatch (August 1, 2012) ......................................................................... 3.3

3.3 Total Regulation Applied (August 1, 2012)..................................................................... 3.3

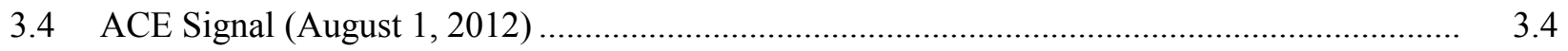

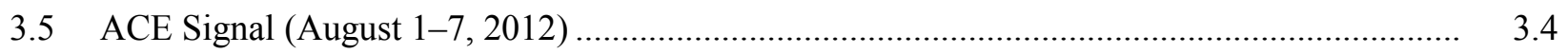

3.6 AGC Units Total Dispatch (August 1-7, 2012) .................................................................. 3.5

3.7 AGC Units Dispatch (August 1-7, 2012) ….................................................................. 3.5

3.8 ACE Distribution (August 1-7, 2012) ...................................................................... 3.6

3.9 ACE Hourly Distribution (August 1-7, 2012) ................................................................. 3.6

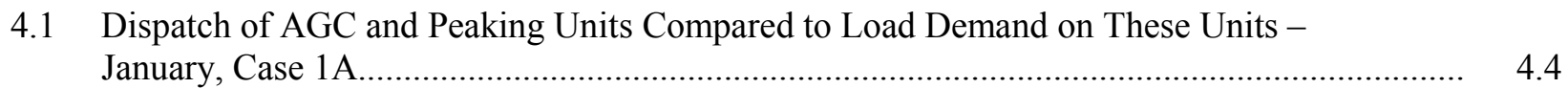

4.2 Total AGC Units Output Compared to their Hourly Schedule - January, Case 1A ................. 4.4

4.3 Dispatch of Each AGC Unit Compared to Hourly Schedules - January, Case 1A .................. 4.5

4.4 Configurations of AGC Units Determined by Unit Commitment - January, Case 1A ............. 4.5

4.5 Available Spinning Reserve and Spinning Reserve Requirement - January, Case 1A ............ 4.6

4.6 Available Regulation Reserves - January, Case 1A .......................................................... 4.6

4.7 Peaking Units Dispatched by the Operator Model in ESIOS - January, Case 1A................... 4.7

4.8 Dispatch of AGC and Peaking Units by ESIOS from January 15 to January 17 that have the Largest Daily Demand Cycles of the Month ........................................................... 4.7

4.9 Dispatch of AGC and Peaking Units by ESIOS from January 12 to January 13 that have Large ACE Over a Prolonged Period.

4.10 Configurations of AGC Units (determined by unit commitment) from January 12 to January 13 that have Large ACE Over a Prolonged Period

4.11 Available Regulation Reserves from January 12 to January 13 that have Large ACE over a Prolonged Period.

4.12 Dispatch of AGC and Peaking Units Compared to Load Demand on These Units January, Case 5A.....

4.13 Dispatch of AGC Units from January 1 to January 3 when Significant Differences were Observed between the Schedule and Load Demand on AGC Units - Case 5A..... 
4.14 Total AGC Units Output Compared to their Hourly Schedule - January, Case 5A ................. 4.11

4.15 Dispatch of Each AGC Unit Compared to Hourly Schedules - January, Case 5A .................. 4.12

4.16 Configurations of AGC Units Determined by Unit Commitment - January, Case 5A ............. 4.12

4.17 Available Spinning Reserve and Spinning Reserve Requirement - January, Case 5A ............ 4.13

4.18 Available Regulation Reserves- January, Case 5A …........................................................ 4.13

4.19 Peaking Units Dispatched by the Operator Model in ESIOS - January, Case 5A.................... 4.14

4.20 Total Deployed AGC Reserves - January, Case 1A............................................................ 4.15

4.21 Deployed AGC Reserves Separated into Load Following and Regulation Components -

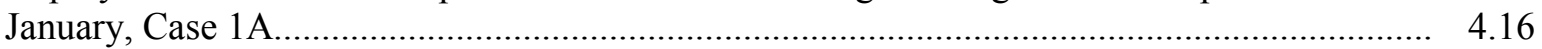

4.22 Maximum Load Following and Regulation Reserve Deployment by Hour - January, Case $1 \mathrm{~A}$.......

4.23 Total Deployed AGC Reserves - January, Case 5A …................................................... 4.17

4.24 Deployed AGC Reserves Separated into Load Following and Regulation Components -

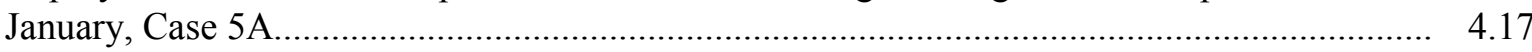

4.25 Maximum Load Following and Regulation Reserve Deployment by Hour - January, Case $5 \mathrm{~A}$

4.26 Regulation Capacity Requirements - Winter, Cases 1A-5A [1] .......................................... 4.18

4.27 Load Following Capacity Requirements - Winter, Cases 1A-5A [1] .................................... 4.19

4.28 ACE Distribution of the Month - January, Case 1A ............................................................ 4.19

4.29 ACE Distribution by Hour - January, Case 1A .................................................................... 4.20

4.30 ACE Distribution of the Month - January, Case 5A …........................................................ 4.20

4.31 ACE Distribution by Hour - January, Case 5A …............................................................... 4.21

4.32 Average ACE in Different PV Cases ............................................................................... 4.22

4.33 CPS2 Performance in Different PV Cases .......................................................................... 4.22

4.34 Duration of Regulation Up Reserve Shortage ..................................................................... 4.23

4.35 Duration of Regulation Down Reserve Shortage ............................................................... 4.23

4.36 Runtime (generator-hour) of Peaking Units dispatched by the Operator Model ...................... 4.24

4.37 Average Ratio of Daily Peak to Bottom of Net Load ............................................................... 4.24

4.38 Operator Manual Dispatch (generator-hour) on Non-Regulation Units ................................. 4.25 


\section{Tables}

2.1. Combined Cycle Generator Characteristic Table .................................................................

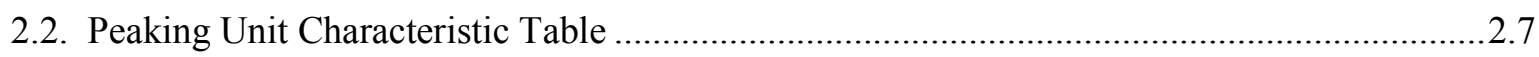

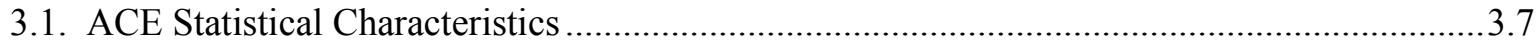

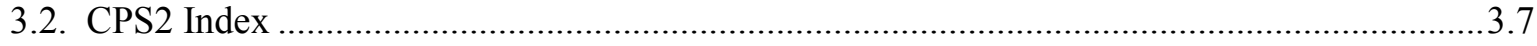

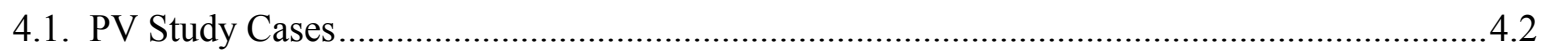

4.2. NV Energy Southern System Load Peaks by Month ............................................................ 4.2

4.3. ACE Statistical Characteristics by Hour - January, Case 1A...............................................4.20

4.4. ACE Statistical Characteristics by Hour - January, Case 5A............................................. 4.21 



\subsection{Introduction}

\subsection{Background}

With over 250 days of sunshine each year, Nevada has the most abundant solar resource in the United States. The state has established a renewable portfolio standard (RPS) under which NV Energy (the electric utility company serving most areas of Nevada) must supply at least 25 percent of the total electricity it sells to retail customers using eligible renewable energy sources by 2025 . In particular, 6 percent of the portfolio requirement must be met through solar energy by 2016 .

To evaluate the operational impacts of integrating large amount of photovoltaic (PV) generation into the system, NV Energy performed a study in 2011 with Navigant Consulting, Inc., Pacific Northwest National Laboratory (PNNL), and Sandia National Laboratories [1]. The study looked at the impacts of PV generation on generator commitment and dispatch, system balancing requirements [2], challenging operating hours [3], and thermal generator cycling and movements for providing balancing services [4][5]. The study showed that the system balancing requirements and generator cycling increase with the penetration of PV generation, and calculated the integration cost caused by curtailment, more reserves, and lower fuel efficiency on thermal generators.

Specific concerns raised by system operators are 1) whether the generation fleet will be able to follow the fast ramps created by the PV output at high penetration levels and 2) whether the system can still comply with North American Electric Reliability Corporation (NERC) control performance standards (CPS). Because existing opeartion modeling tools can only perform dispatch simulations hourly and do not have the necessary time granularity to answer the above questions, a simplified approach was used in the 2011 study to identify the challenging operation hours; that is, when the online generators do not meet flexibility requirements to accommodate the PV variations [3]. The study concluded that the NV Energy fleet is capable of meeting balancing requirements during these challenging hours with appropriate redispatch by operators. Regardless of that finding, more detailed studies on the system intra-hour dispatch and automatic generation control (AGC) ${ }^{1}$ were demanded to verify the results and provide operators with better information on what to expect, and give them more confidence in handling intra-hour variability.

In general, existing tools for power system operation modeling have focused on hourly unit commitment of generating resources. With increasing penetration of variable renewable generation (VG), such as wind and PV, balancing generation and load within the hour has become more and more challenging. Operators often find themselves scrambling to seek additional resources to cover the shortage or outlets to send the excess created by VG. The capability to look into the intra-hour balancing processes is of great value to these systems.

New technologies have been proposed to increase system flexibility, such as energy storage and demand response (DR). Control approaches in both AGC and real-time dispatch (RTD) ${ }^{2}$ are being improved to accommodate these new resources. Market rules to compensate for better regulation performance are also under development as required in the newly issued Federal Energy Regulatory

\footnotetext{
${ }^{1} \mathrm{AGC}$ is an automated mechanism for adjusting the power output of multiple generating resources of an electric power system, in response to instantaneous changes in the load

${ }^{2}$ RTD is an operation process for dispatching generating resources at a fixed 5 to 15 - minute interval to follow the trend of real-time load
} 
Commission Order 755. ${ }^{1}$ Models and software tools capable of performing intra-hour dispatch and AGC simulations will help in testing these control approaches and in evaluating new market rules.

\subsection{Objectives}

The goal of this project is to develop a software program that can perform intra-hour dispatch and AGC simulation for the NV Energy southern system, and then use the program to study the impacts of PV generation on system intra-hour operations and control performance. With detailed and realistic depictions of generator dispatch, questions such as whether the existing generation fleet is flexible enough to follow PV variations and how many MW of AGC reserves would be sufficient can be answered. The program, named Electric System Intra-Hour Operation Simulator (ESIOS), contains modules that simulate computerized AGC and RTD functions balancing generation and load in real time, as well as an operator model that mimics manual actions to maintain system reserves. Actual dispatch of AGC generators and control performance under various PV penetration levels can be predicted by running the simulator. On the other hand, ESIOS also is designed to be a test bed for new storage and DR control methods and market rules.

This report describes the functions of the ESIOS and presents the study results on the PV generation impacts on real-time operations in the NV Energy southern system. If provided with necessary load, generation and generator characteristics data, ESIOS also can be used to perform similar simulations and assessments for other systems.

\subsection{Organization of the report}

The rest of this report is organized as follows. Chapter 2 describes the function modules of the simulator, which includes the model of AGC logic and economic dispatch, AGC generator models, and operator action model. Chapter 3 shows the validation results comparing ESIOS dispatch with NV Energy system historical data. Chapter 4 presents the results by running ESIOS using data from five PV case studies developed in the 2011 PV integration study. Chapter 5 presents our conclusions.

\footnotetext{
${ }^{1}$ Frequency Regulation Compensation in the Organized Wholesale Power Markets, Order No. 755, 76 FR 67260 (October 31, 2011), Federal Energy Regulatory Commission Statistics and Regulations 31,324 (2011).
} 


\subsection{Intra-Hour Dispatch and AGC Simulator}

\subsection{Function Modules}

ESIOS contains three main modules: 1) a preprocessor, 2) the intra-hour dispatch simulator, and 3 ) a post-processor. The preprocessing module removes outliers, fixes missing data, and performs interpolation if necessary to produce high-time-resolution data for conventional generation, variable generation, load, and interchange. Users can choose the time resolution of the data. Usually, a resolution of 1 minute is deemed sufficient for the study of intra-hour variability of power systems because aggregated load and VG changes in a system are insignificant and tolerable within a minute.

Time series data from the preprocessing module are then sent to the dispatch simulator to perform AGC and RTD simulations step by step, until the completion of the entire time series. Models of generators or other resources subject to AGC and RTD are needed to provide resource capability and constraints information for the dispatch simulator. Operator actions in addition to computerized AGC and RTD processes are simulated as necessary; for example, when the system does not have sufficient balancing resources, operators may need to start a peaking unit or manually re-dispatch nonregulating units. Real-time forecasts of VG and load, as separate functions, provide references for RTD and prediction of reserve requirements used by the operator model.

Most function blocks in the dispatch simulator can be enabled or disabled depending on the way a specific system works or the objective of the simulation. They also can be set to run at different time intervals (e.g., AGC every minute, RTD every 5 minutes, and operator model every 10 minutes). Parameters of each function block are currently set through the initialization file.

Figure 2.1 is a block diagram depicting the dispatch simulator module. The RTD function is not simulated as a separate process for the NV Energy southern system. Economic dispatch in real time is performed inside the AGC function.

The post-processing module takes intra-hour dispatch and AGC results to calculate system control performance, deployed reserves, number of peaking units, running hours, etc.

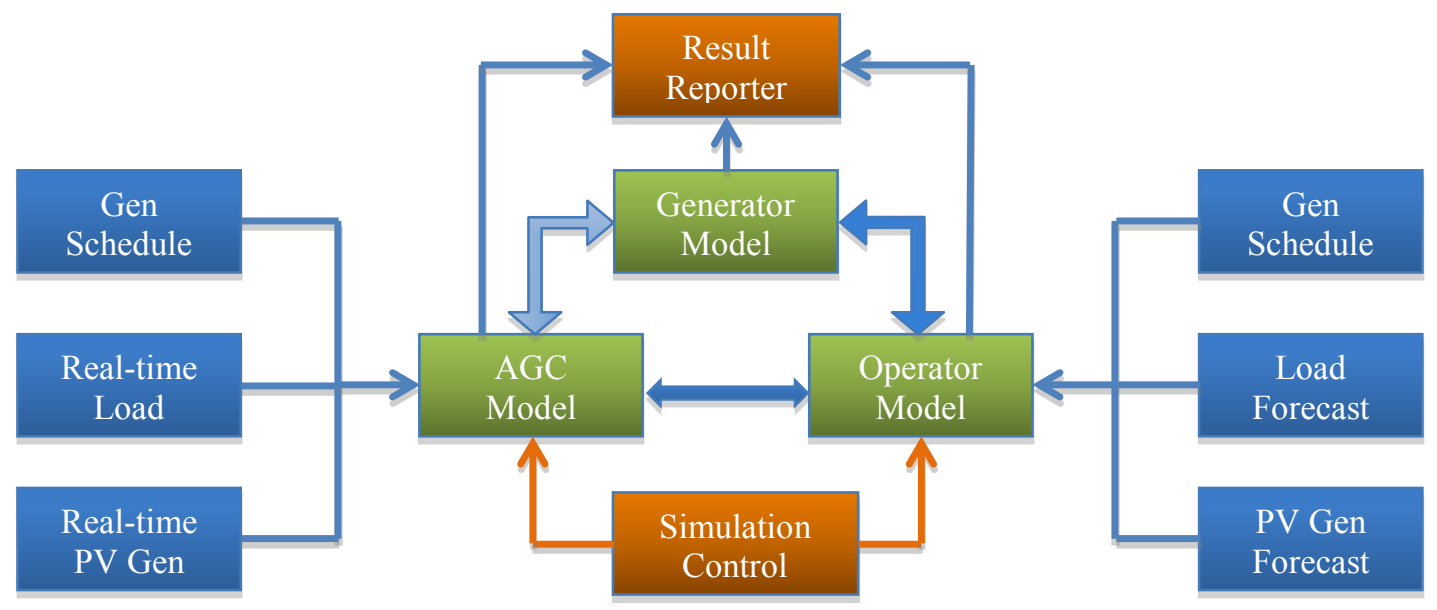

Figure 2.1. Block Diagram of the Dispatch Simulator Module 


\subsection{Automatic Generation Control}

\subsubsection{Methodology}

The objective of AGC is to balance load and generation in real time to the degree required by the NERC CPS. The target of AGC is to contain the area control error (ACE). ACE has two major components: 1) unscheduled interchange and 2) the response of the balancing area to frequency deviations. In this work, because only one balancing area is considered in the simulation, the difference between area generation ${ }^{1}$ and area load is an accurate target for AGC to minimize.

Figure 2.2 and Figure 2.3 show the diagram for real-time dispatch and AGC, respectively. As shown in Figure 2.2, real-time dispatch is performed based on the difference between the hourly generation schedule and real-time net area load forecast. Net area load is the area load minus variable generation. Through economic dispatch of the generation resources participating in real-time dispatch (also called load-following resources), adjustments to the hourly schedule of these resources can be calculated, which is called the real-time dispatch signal. The adjusted schedule is called the real-time schedule. The way in which hourly schedules are determined, how often RTD is performed, and the look-ahead time of the realtime forecast can all be configured according to the system being modeled.

As shown in Figure 2.3, the difference between net area load and total area generation determines the raw ACE. The control ACE is derived from an ACE filter and a controller applied to the raw ACE, which is then allocated to each AGC generator based on economics, ramp properties, or a pre-calculated ratio. Therefore, the setting of generator operation point is the sum of real-time schedule and the regulation signal. Combined with information from Figure 2.2, we have:

$$
\text { Operation Point }=\text { Hourly Schedule }+ \text { Realtime Dispatch Signal }+ \text { Regulation Signal }
$$

The actual outputs of AGC generators are based on the operation point settings subject to the properties and constraints applied by the generation plant model. Slow governor dynamics, ramping constraints, and generation control errors can be simulated in the generation plant model if desired. The total area generation depicted in Figure 2.3 includes the effects of area imports and exports. When no separate RTD process is simulated (which is the case for the NV Energy southern system), real-time schedule is replaced by hourly schedule of generation resources before adding the regulation signal, and economic dispatch is performed inside the AGC controller.

Design of AGC logic is of great practical value and has been a research topic for decades [6]-[10]. While the main objective of AGC is balancing generation and load in real time, the algorithm also has goals of minimizing the number of AGC pulses, the number of AGC pulse reversals, etc., to reduce generator wear resulting from regulation service. The following two sections will describe the ACE filter and controller adopted in the ESIOS program to simulate the NV Energy southern system [11]. Other AGC algorithms also can be used in ESIOS to model a different system or to simply test their performance.

\footnotetext{
${ }^{1}$ In this report the term "area generation" refers to all generation used to meet the balancing area's load, which includes the net interchange as well as generation inside the balancing area.
} 


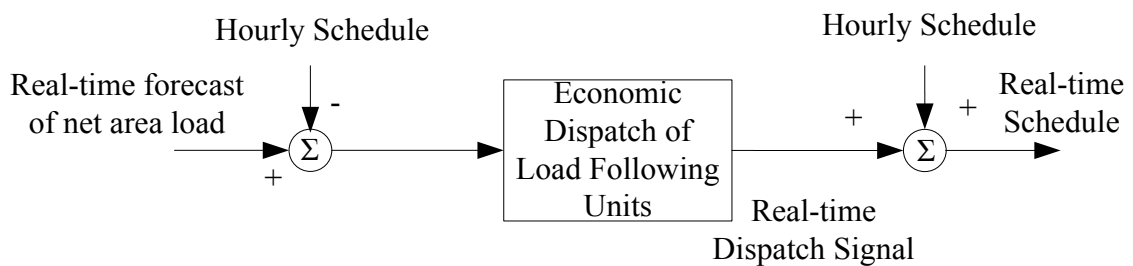

Figure 2.2. Diagram for Real-Time Dispatch Simulation

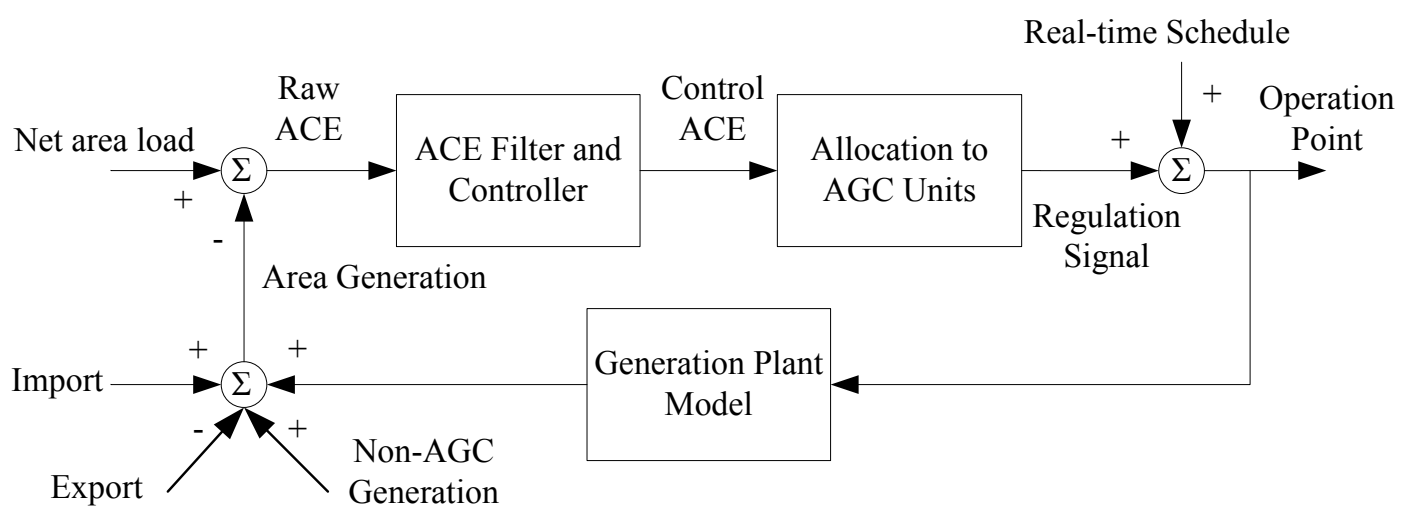

Figure 2.3. Diagram for AGC Simulation

It should also be pointed out that exactly replicating the AGC in the NV Energy southern system was not deemed necessary by the study team, because AGC logic normally can be tuned to achieve a wide range of desired control performance within the AGC generators' capability. A representative AGC implementation is sufficient for the study of generator fleet flexibility affected by both generator characteristics and unit commitment and scheduling results. If testing the AGC logic is the main goal of simulation, duplicating the exact AGC logic in ESIOS becomes necessary.

\subsubsection{ACE Filtering}

A low-pass filter is used to smooth the raw ACE to remove measurement noise and reduce unnecessary movement of AGC generators. The filter design in ESIOS mimics approximately the one in the NV Energy southern system and can be represented as the following.

$$
\begin{gathered}
\text { Raw ACE: ACE_t }=\text { generation_t }- \text { load_t } \\
\text { Smoothed ACE: SACE_t }=(1-a) * \text { SACE_(t-1) }+a^{*} \text { ACE_t }
\end{gathered}
$$

In Equation (2.3), $a$ is a variable ranging between 0 and 1, which specifies the weight of raw ACE from the present time step. The value of $a$ can be adjusted to control how fast AGC resources follow the raw ACE.

\subsubsection{ACE Controller}

The smoothed ACE (SACE) at the output of ACE filter is then processed to generate the control ACE before allocation among AGC resources. The control ACE is calculated by: 


$$
\mathrm{CACE}=\mathrm{RACE}+\mathrm{EACE}
$$

in which RACE is called regulation ACE and EACE emergency assist ACE. RACE and EACE are determined by applying different gains on the smoothed ACE according to the ACE control regions described below:

1. Normal: When abs(SACE) $<$ REGDB, regulation and emergency assist ACE are both 0 ; that is, $\mathrm{RACE}=0$ and $\mathrm{EACE}=0$

2. Regulation: When REGDB $<=\operatorname{abs}(\mathrm{SACE})<=\mathrm{EADB}$,

$$
\begin{aligned}
& \mathrm{RACE}=(\mathrm{SACE}-\mathrm{REGDB}) * \mathrm{~K} \_\mathrm{R}, \text { if SACE }>0 ; \\
& \mathrm{RACE}=(\mathrm{SACE}+\mathrm{REGDB}) * \mathrm{~K} \_\mathrm{R}, \text { if } \mathrm{SACE}<0 ; \text { and } \\
& \mathrm{EACE}=0
\end{aligned}
$$

3. Emergency assist: When abs(SACE) $>$ EADB,

$$
\begin{aligned}
& \mathrm{RACE}=(\mathrm{EADB}-\mathrm{REGDB}) * \mathrm{~K} \_\mathrm{R}, \mathrm{EACE}=(\mathrm{SACE}-\mathrm{EADB}) * \mathrm{~K} \_\mathrm{A}, \text { if } \mathrm{SACE}>0 \text {; and } \\
& \mathrm{RACE}=-(\mathrm{EADB}-\mathrm{REGDB}) * \mathrm{~K} \_\mathrm{R}, \mathrm{EACE}=(\mathrm{SACE}+\mathrm{EADB}) * \mathrm{~K} \_\mathrm{A}, \text { if } \mathrm{SACE}<0
\end{aligned}
$$

in which REGDB is the regulation dead band (e.g., $12 \mathrm{MW}$ ), EADB is the emergency assist dead band (e.g., $25 \mathrm{MW}$ ), and $\mathrm{K} \_\mathrm{R}$ and $\mathrm{K} \_\mathrm{A}$ are the proportional coefficients for the regulation and emergency assist regions, respectively. Example values for $\mathrm{K} \_\mathrm{R}$ and $\mathrm{K} \_\mathrm{A}$ are 0.8 and 1.0.

Values of the regulation and emergency dead bands, coefficients K_R and K_A can be tuned to achieve the desired control performance. Figure 2.4 shows the ACE distribution and time series for a $\mathrm{PV}$ case with a regulation dead band of $12 \mathrm{MW}$ and an emergency assist dead band of $25 \mathrm{MW}$. Figure 2.5 shows the similar plots with these two dead bands set to $24 \mathrm{MW}$ and $50 \mathrm{MW}$, respectively. As can be seen, the dead bands have significant impact on the value of ACE.

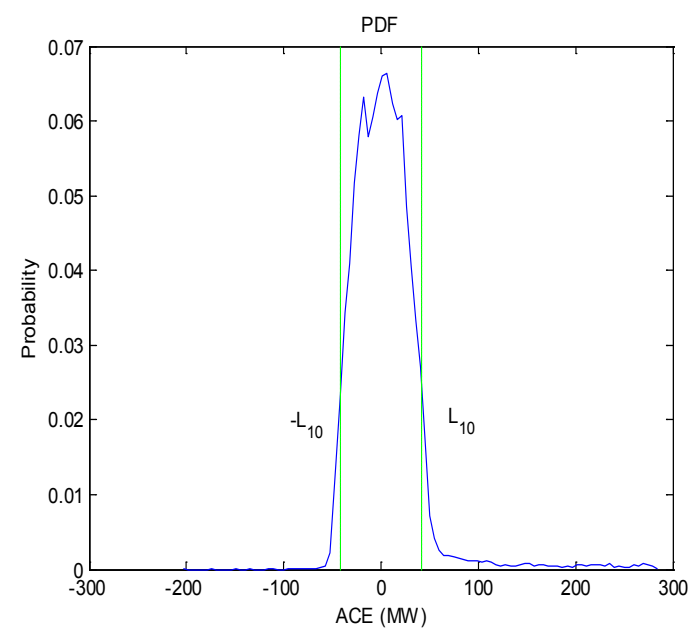

(a)

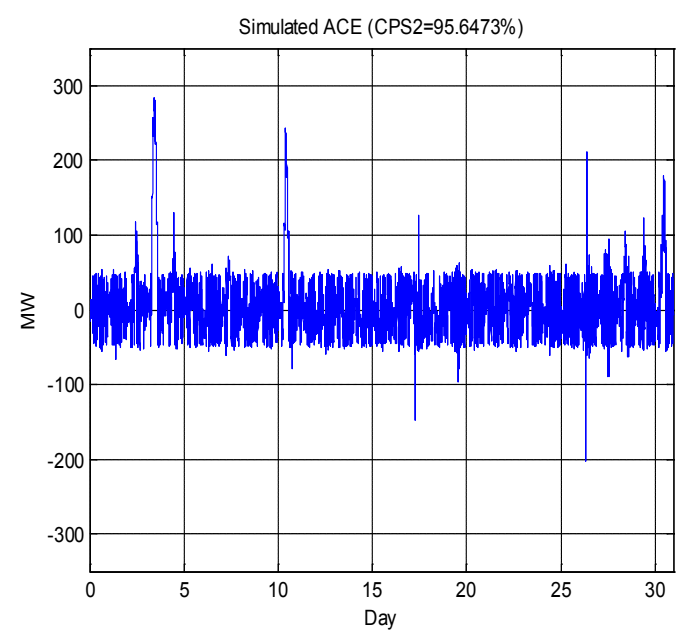

(b)

Figure 2.4. $\mathrm{ACE}$ Distribution and Time Series with $\mathrm{REGDB}=12 \mathrm{MW}$ and $\mathrm{EADB}=25 \mathrm{MW}(a=0.6$, K_R $=0.8$, K_A $=1.0$, March, PV Case 5A) 


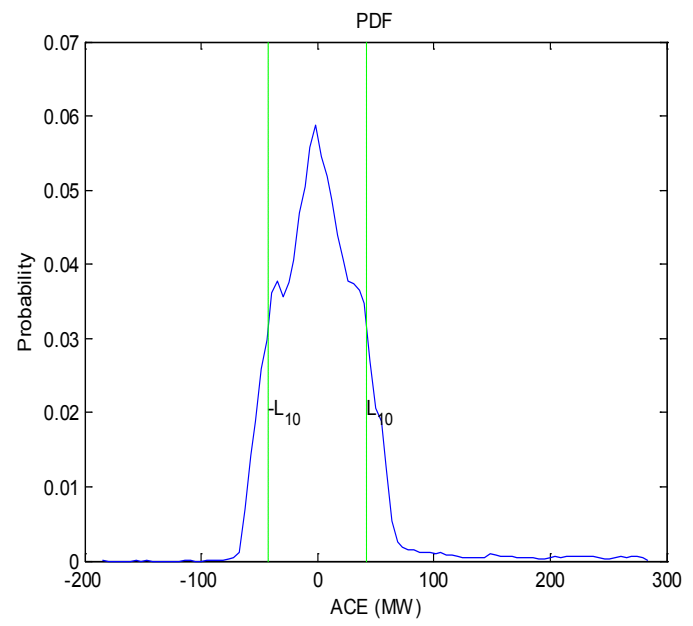

(a)

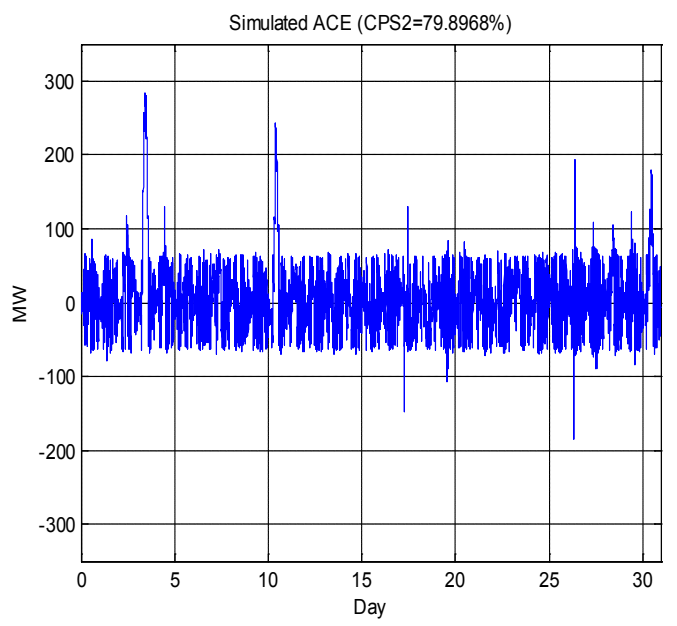

(b)

Figure 2.5. $\mathrm{ACE}$ distribution and time series with $\mathrm{REGDB}=24 \mathrm{MW}$ and $\mathrm{EADB}=50 \mathrm{MW}(a=0.6$, K_R $=0.8$, K_A $=1.0$, March, PV Case 5A)

While the dead bands can be narrowed to improve ACE, dead bands that are too small will cause unnecessary adjustments on the AGC units and increase wear and tear. Figure 2.6 and Figure 2.7 show the ACE and AGC unit movements when regulation dead bands are $12 \mathrm{MW}$ and $0 \mathrm{MW}$, respectively. The removal of REGDB results in continuous changes on the outputs of AGC units, which is less desirable.
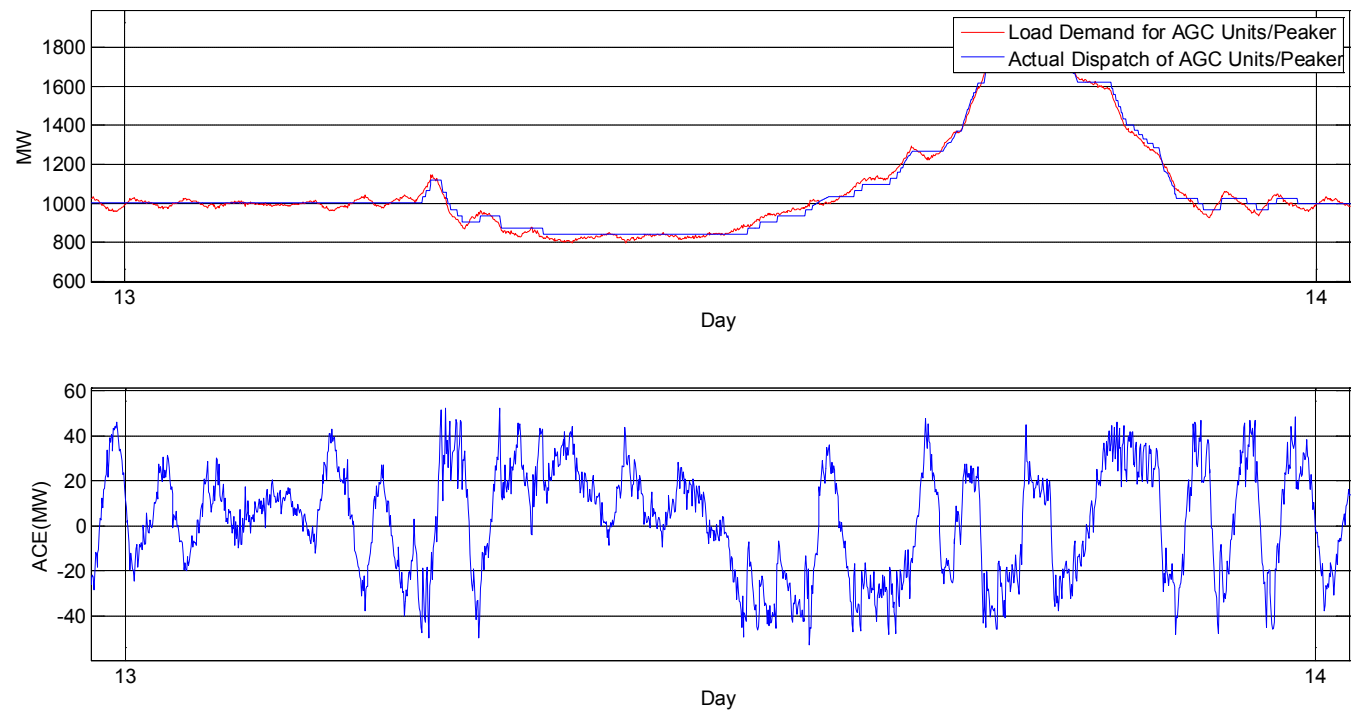

Figure 2.6. $A G C$ unit movement and $A C E$ on March 14 with $R E G D B=12 \mathrm{MW}(E A D B=25 \mathrm{MW}$, $a=0.6, \mathrm{~K} \_\mathrm{R}=0.8, \mathrm{~K} \_\mathrm{A}=1.0, \mathrm{PV}$ Case $5 \mathrm{~A}$ ) 

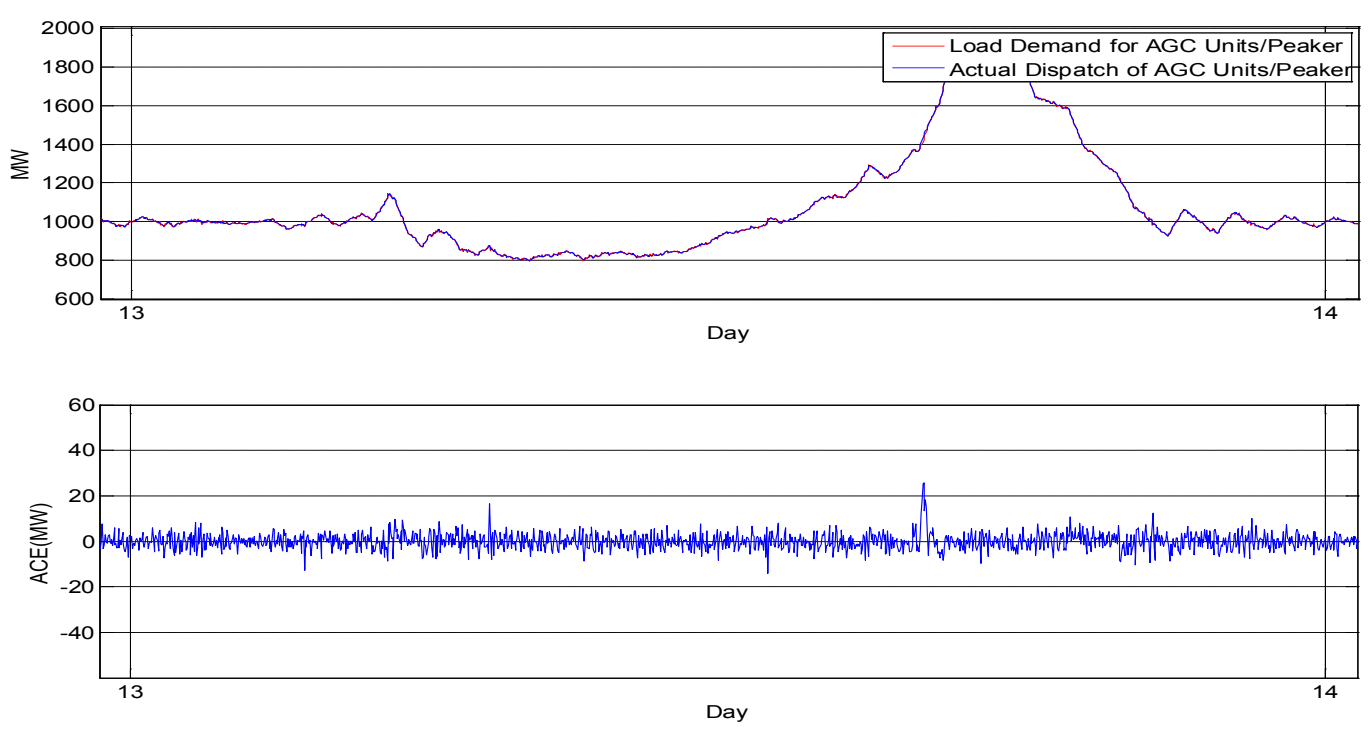

Figure 2.7. $\mathrm{AGC}$ unit movement and $\mathrm{ACE}$ on March 14 with $\mathrm{REGDB}=0 \mathrm{MW}(\mathrm{EADB}=25 \mathrm{MW}$, $a=0.6, \mathrm{~K} \_\mathrm{R}=0.8, \mathrm{~K} \_\mathrm{A}=1.0, \mathrm{PV}$ Case $5 \mathrm{~A}$ )

\subsection{Models of AGC Resources}

Models that realistically represent the capability and constraints of AGC resources are critical for the simulation of intra-hour dispatch and AGC. Important characteristics include startup time, shutdown time, minimum run time, ramp rate, range of regulation capacity, and minimum and maximum power output, etc. For natural gas combined-cycle (CC) units, the characteristics under each configuration need to be carefully modeled. For storage devices controlled by AGC, energy capacity limits need to be considered.

Two types of AGC resources are included in the ESIOS modeling for the NV Energy southern system: 1) CC units and 2) peaking units (simple cycle gas turbines that colloquially are referred to as "peakers"). The CC units are the two-by-one type (two combustion turbines [CT], plus one heat recovery steam turbine [ST]). When needed, the CTs also can fire their duct burners (DB) to increase generation. Therefore, the $\mathrm{CC}$ units can have seven different configurations. We created a characteristic table for each CC unit for the AGC module, an example of which is shown in Table 2.1. For economic reasons, most of the time the CC units only run in the four configurations shown in Table 2.1; therefore, other configurations were not considered. When modeling the CC units in the NV Energy southern system, the regulation range is set to zero once the DBs are started. The AGC model will not be able to adjust generation of CC units under DB modes. However, the operator model can still dispatch them "manually." Characteristics of all other AGC generators simulated in the NV Energy southern system are listed in Appendix A (actual generator names were replaced with CC Units A through E). 
Table 2.1. Combined Cycle Generator Characteristic Table

\begin{tabular}{lcccccccc}
\hline & & Configuration & Configuration & & & & & \\
Unit Name & Unit ID & Name & Name Alias & Pmin & Pmax & Rgmin & Rgmax & Ramp Rate \\
\hline CC Unit A & 1 & 1 & $1 \times 1$ & 150 & 250 & 150 & 250 & 8 \\
CC Unit A & 1 & 2 & $2 \times 1$ & 250 & 500 & 250 & 500 & 16 \\
CC Unit A & 1 & 3 & $2 \times 1,1$ DB & 500 & 525 & 0 & 0 & 4 \\
CC Unit A & 1 & 4 & $2 \times 1,2$ DB & 525 & 550 & 0 & 0 & 4 \\
\hline
\end{tabular}

Peaking units are modeled with parameters including startup time, shutdown (ramp down) time, minimum run time, and power capacity limits. An example of peaking unit characteristics is shown in Table 2.2.

Table 2.2. Peaking Unit Characteristic Table

\begin{tabular}{ccccccc}
\hline Unit Name & $\begin{array}{c}\text { Unit } \\
\text { Type }\end{array}$ & $\begin{array}{c}\text { Pmin } \\
(\mathrm{MW})\end{array}$ & $\begin{array}{c}\text { Pmax } \\
(\mathrm{MW})\end{array}$ & $\begin{array}{c}\text { Startup } \\
\text { Time }(\mathrm{min})\end{array}$ & $\begin{array}{c}\text { Shutdown } \\
\text { Time }(\mathrm{min})\end{array}$ & $\begin{array}{c}\text { Min Runtime } \\
(\mathrm{min})\end{array}$ \\
\hline Peaker.X & Peaker & 35 & 57 & 10 & 10 & 30 \\
\hline
\end{tabular}

\subsection{Operator Dispatch Actions}

\subsubsection{Methodology}

The objective of the operator model is to simulate the manual dispatch actions performed by operators that are necessary to maintain required operating reserves and control performance. Because of the variability and uncertainty with load and variable generation, the computerized balancing processes (AGC and RTD) may not be capable of following real-time changes of net load, especially in high VG penetration cases. The operator model in ESIOS monitors regulation and spinning reserves available on the system, and adjusts them by starting peaking units and re-dispatching non-regulating AGC units. Other functions, such as the commitment of additional large generators or de-commitment of online generators, could also be performed in the operator model but currently are not implemented.

The operator model estimates the average regulation and spinning reserves available during a specified time period. ${ }^{1}$ When regulation up (RegUp) reserve is estimated to be insufficient, generators without regulation capability are raised by the amount of shortage until their maximum capacity is reached; when regulation down (RegDn) reserve is insufficient, either non-regulation generation has to be lowered or a CC generator has to switch from $2 \times 1$ to $1 \times 1$ mode. Considering the restart of a CT takes nearly 2 hours, turning off CT simply to increase RegDn reserve for a short period may not be prudent. Therefore, the program currently only lowers non-regulation generators to resolve the shortage of RegDn reserve. Non-regulation generators that have been dispatched off schedules by the operator model will be

\footnotetext{
${ }^{1}$ In principle, the spinnng reserve requirement should be satisfied in every moment in actual operations. The average available spinning reserve is used in the simulation to compare against the requirement to avoid frequent dispatch of peaking units caused by instantaneous reserve shortages.
} 
checked regularly to determine whether regulation reserves would be sufficient if these generators returned to their operating schedules.

Peaking units are turned on when a shortage of spinning reserve is predicted, or when RegUp reserve is insufficient and there is no head-room on non-regulation units. On the other hand, peaking units are turned off when they are not needed so that system efficiency can be improved and RegDn reserve is increased simultaneously.

The following functions are included in the operator model:

- Spinning reserve check and adjustment

- Turn on peaking units if spinning reserve is insufficient. Spinning reserve requirement is determined dynamically based on the output of the largest online generator and the share and obligation of the balancing authority (BA) within the reserve sharing pool.

- RegUp reserve check and adjustment

- Increase Non-Reg generation when more RegUp is needed, or return Non-Reg generation back to schedule when RegUp is sufficient.

- Turn on peaking units if Non-Reg generation adjustment is insufficient to resolve RegUp shortage.

- RegDn reserve check and adjustment

- Decrease Non-Reg generation when RegDn is needed, or return Non-Reg generation back to schedule when RegDn is sufficient.

- Turn off peaking units when conditions allow.

\subsubsection{Startup and Shutdown of Peaking Units}

The operation of peaking units for the purpose of maintaining regulation and spinning reserves is described in details below.

\section{Available reserve calculation}

- Available RegUp at time $t$ is calculated by:

$$
R_{U p_{t}}=P_{\text {Reg_max }_{t}}-P_{\text {Reg_Gen }_{t}},
$$

where $P_{\text {Reg_max }}$ is the maximum regulation units capacity available at time $t$, and $P_{\text {Reg_Gen }}$ is the total regulation units output at time $t$.

- Available RegDn at time $t$ is calculated by:

$$
R_{D n_{t}}=P_{\text {Reg_min }_{t}}-P_{\text {Reg_Gen }_{t}},
$$

where $P_{\text {Reg_min }}$ is the minimum regulation units capacity available at time $t$.

- Available spinning reserve at time $t$ is calculated by:

$$
S_{t}=P_{\max _{t}}-P_{\text {Gen }_{t}}
$$


where $P_{\text {max }}$ is the maximum generation capacity of all online units available at time $t$, and $P_{\text {Gen }}$ is the total online generation at time $t$. 


\section{Peaking unit activation}

The available reserves calculated from step 1 will be compared to the corresponding requirements. If a shortage of regulation reserves (RegUp or RegDn) is detected, the operator model will first check if non-regulating AGC units can be adjusted to increase the reserves. Manually moving nonregulating units upward will increase RegUp. Moving them downward will help with RegDn. If there is no head room on non-regulating AGC units but still more RegUp is needed, then peaking units need to be started:

$$
\text { If } R_{U p_{t}}<R_{U p_{\min }} \text { and } L_{t}<L F_{t}^{x} \text {, then activate peaking unit } \mathrm{N} \text {, }
$$

where $R_{U p_{\min }}$ is the required RegUp reserve, $L_{t}$ is the net load at time $t$, and $L F_{t}^{x}$ is the net load forecast for the next $x$-minute interval produced at time $t$. This condition means that, if available RegUp reserve is less than the requirement and the load is increasing, then a peaking unit is started. $\mathrm{N}$ is a peaking unit identifier (ID). Peaking units are ordered according to production cost, and started from the cheapest one. The value assigned to $x$ can range from 0 to 0.5 (hours) to avoid frequent on/off operation of units.

If spinning reserve is found insufficient, peaking units will be started directly:

$$
\text { If } S_{t}<S_{\min } \text { and } L_{t}<L F_{t}^{x} \text { then activate peaking unit } \mathrm{N} \text {, }
$$

where $S_{\min }$ is the required spinning reserve. $S_{\min }$ is calculated as the following:

$$
S_{\text {min }}=\max \left(\frac{1}{2} P_{\text {Gen }_{k_{t}}}+\left(P_{\text {Gen }_{k_{\text {max }}}}-P_{\text {Gen }_{k_{t}}}\right)-S_{\text {Share }}, S_{\text {Obligation }}\right), k=1 \ldots M,
$$

where $P_{G e n_{k_{t}}}$ is the output of the $k$ th online generator, $P_{G e n_{k_{\max }}}$ is the maximum output of this generator, $S_{\text {Share }}$ is the spinning reserve share for NV Energy from a reserve sharing pool, $S_{\text {Obligation }}$ is the obligated amount that NV Energy needs to provide to the pool, and M is the total number of online generators. Equation (2.10) means the total system spinning reserve needs to be larger than half of any online generator's output plus the reserve it carries, minus the share the BA gets from the reserve sharing pool. It also needs to be larger than the reserve obligation that the BA needs to provide to the pool in case of emergency in other systems in the pool.

\section{Peaking unit deactivation}

The following conditions will be constantly checked by the operator model. Peaking units will be shutdown to improve system efficiency if these conditions are satisfied simultaneously:

$$
\begin{gathered}
t-t_{\text {tart }_{N}} \geq \text { MinRuntime }_{N} \\
H S_{\text {NextHour }_{N}}=\text { off } \\
R_{U p_{t}}-P_{N}>R_{U p_{\text {min }}} \\
S_{t}-P_{N}>S_{\text {min }}, \text { and } \\
L_{t}>L F_{t}^{x}
\end{gathered}
$$

where $t_{\text {start }}$ is the time when peaking unit $\mathrm{N}$ is started, MinRuntime ${ }_{N}$ is the minimum runtime of the peaking unit, $H S_{\text {NextHour }_{N}}$ is its schedule in the next hour, and $P_{N}$ is its current power output. These conditions state that when a peaking unit is to be turned off, it must have run longer than its 
minimum runtime, the next hour's schedule must be off, reserve requirements are still satisfied after it is off, and load shall be decreasing.

Alternatively, available reserves $R_{U p_{t}}, R_{D n_{t}}$, and $S_{t}$ can be calculated for the period from time $t-k 1$ to $t+k 2$ and then compared to the predefined reserve requirement in the above steps. Parameters $k 1$, $k 2$ and forecast horizon $x$ can all be configured to achieve optimal operation performance.

The process is illustrated in Figure 2.8 using RegUp as an example.

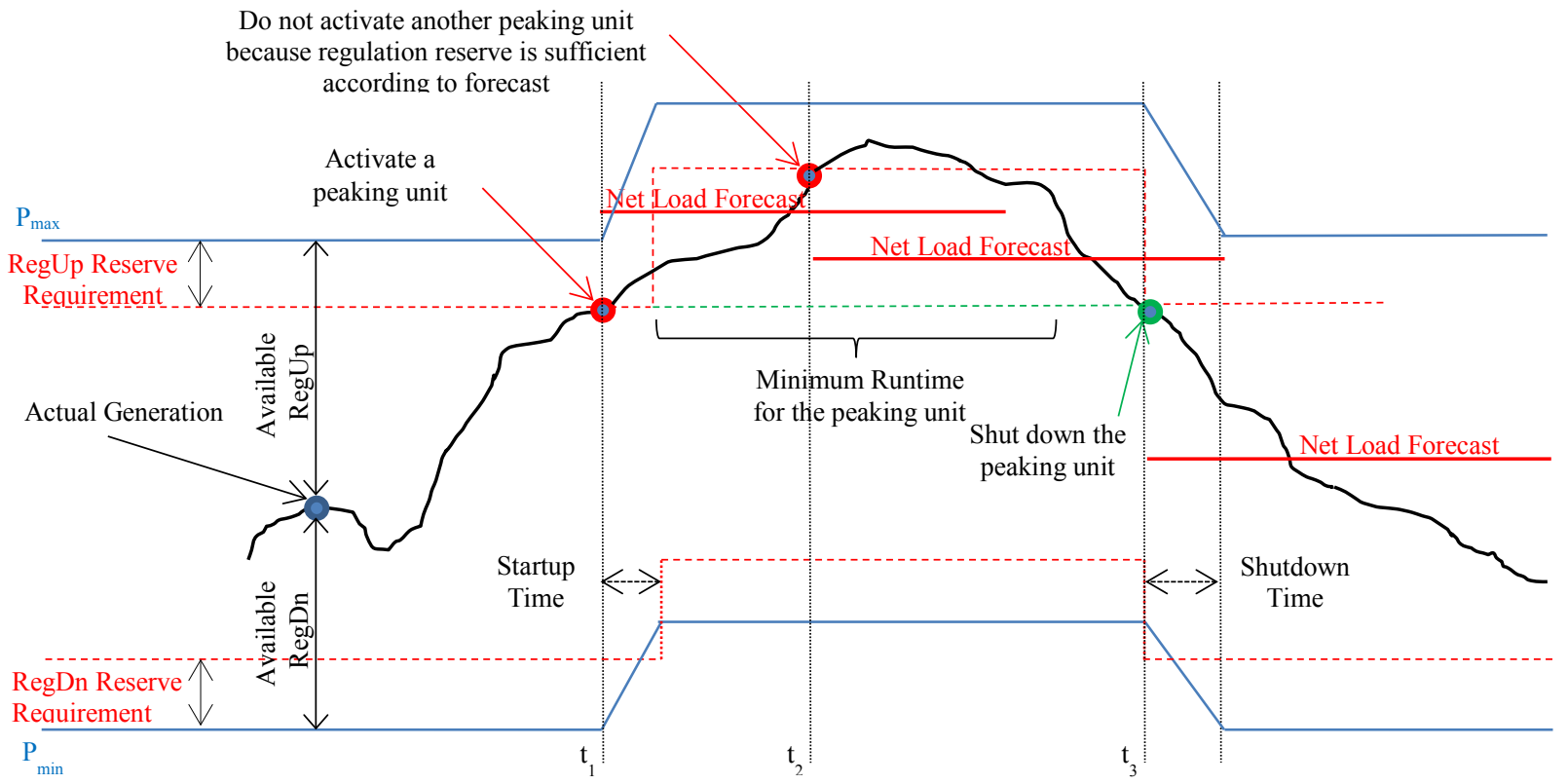

Figure 2.8. Peaking Unit Operation Approach

Operator actions for the peaking units shown in Figure 2.8 are described below:

- Time $t_{1}$. Available RegUp reserve becomes less than the amount required and net load forecast for the next $\mathrm{X}$-minute interval is higher than the actual net load at the moment (i.e., net load is increasing). Therefore, peaking unit 1 is started. Both $\mathrm{P}_{\max }$ and $\mathrm{P}_{\min }$ of online generation are increased.

- Time $t_{2}$. Available RegUp reserve becomes less than its requirement again, but net load forecast for next x-minute interval is lower than the actual net load currently (i.e., net load is decreasing). Therefore, there is no need to start another peaking unit.

- Time $t_{3}$. RegUp reserve becomes sufficient even after the peaking unit is shutdown, and net load forecast is lower than the actual net load at the moment (i.e., net load is decreasing). The time since the peaking unit is started has exceeded its minimum runtime. Therefore, the peaking unit can be shut down. $\mathrm{P}_{\max }$ and $\mathrm{P}_{\min }$ of online generation are reduced consequently. 


\subsubsection{Statistics of Operator Actions}

The manual dispatch actions by operators, such as starting peaking units and re-dispatching nonregulation generators, are only performed when system spinning and regulation reserves are insufficient. Therefore, the number of such actions is an indication of the reserve adjustments needed and the operation challenge for a particular study case. In the meantime, because the intra-hour dispatch and AGC simulation is carried out based on a set of hourly generation schedules, the need of manual dispatch also is greatly affected by the quality of unit commitment and scheduling results; that is, how well the generators placed online can meet the capacity and flexibility required for following load and VG.

The graphs in Figure 2.9 compare the starts of peaking units in the same month for a low (Case 1A) and a high (Case 5A) PV penetration case. The high PV case demands more frequent starts and more run time of peaking units in the month shown. ${ }^{1}$

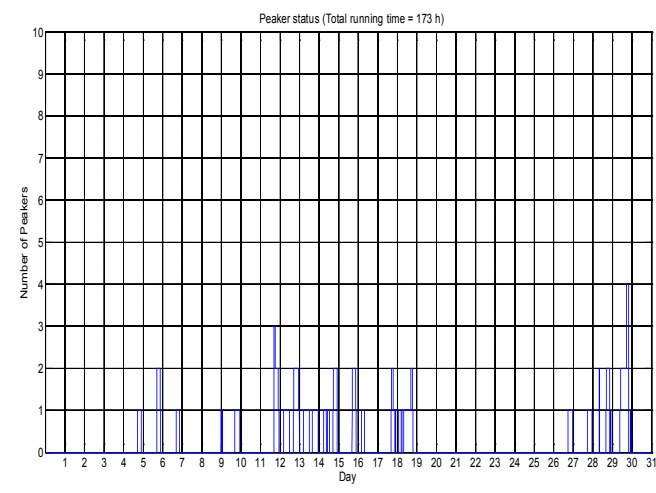

(a) Case 1A (low PV) January

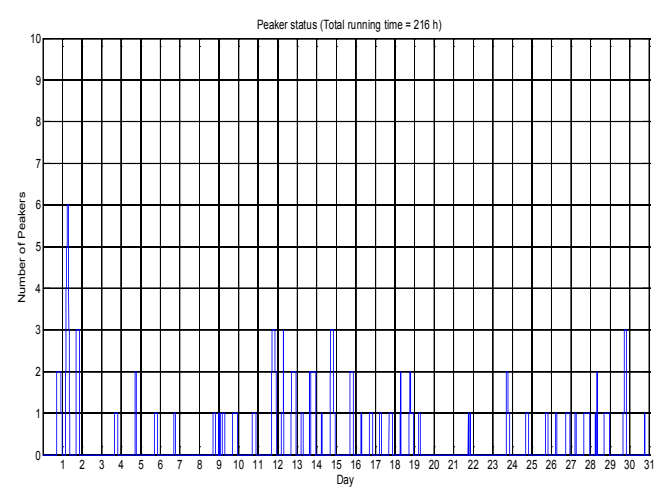

(b) Case 5A (high PV) January

Figure 2.9. Startups and Runtime of Peaking Units in a Low and a High PV Penetration Case

\footnotetext{
${ }^{1}$ This is not generally true for other months because the dispatch of peaking units is affected by the shapes of both load and PV generation. The combined effect changes depending on time of the year, following seasonal load and PV generation patterns. Please refer to Section 4.3 for more details on general observations from the simulation results.
} 


\subsection{Simulator Validation}

\subsection{Simulation of NV Energy Southern System AGC}

\subsubsection{Validation Data}

One week (August 1-7, 2012) of actual NV Energy southern system AGC data were used to validate the simulator. The data set includes actual load, generation schedule, export/import information, 4-second generator output and actual ACE, and generation fleet characteristics. Real-time adjustments by operators to satisfy reliability requirements or fulfill inter-system transactions were not accounted due to the difficulty in collecting relevant data. Generation schedule (day-ahead and hour-ahead) and generator characteristics $\left(\mathrm{P}_{\min } / \mathrm{P}_{\max }\right.$, ramp rate, and heat rate) are used as the input of the simulation tool. AGC generators output and ACE values are compared between actual data and simulations for the simulator validation.

\subsubsection{Simulation Assumptions}

During the simulation process, the following assumptions were used:

- Economic dispatch was performed every minute in AGC.

- AGC units (units used for regulation) include CC Units A through E and X.

- Non-AGC units followed exactly their hourly schedules, and 20-minute ramps were used between the schedules of two neighboring hours.

- Interchanges equaled exactly their hourly schedules and followed the 20-minute ramps between neighboring hours.

- AGC units can operate within the $\mathrm{P}_{\min }-\mathrm{P}_{\max }$ interval.

- NERC CPS $2^{1}$ calculation for the NV Energy southern system uses an $\mathrm{L}_{10}{ }^{2}$ of $42.23 \mathrm{MW}$.

The operator model in the simulator was not enabled in the validation process for the lack of corresponding data as well as simplification.

\subsubsection{Validation Approaches}

The following approaches have been applied to validate results produced by the simulator:

1. Visual Inspection - Comparison of the following actual (recorded) data and simulated data:

- Generation dispatch curves

- ACE curves

\footnotetext{
${ }^{1}$ The NERC CPS2 calculation is a NERC control performance measure designed to bound ACE 10-minute averages and to limit excessive unscheduled power flows that could result from large ACEs [12]

${ }^{2} \mathrm{~L}_{10}$ is the bound for ACE 10-minute averages in CPS2 calculations, above which a violation is counted for a tenminute period
} 
2. Statistical Analysis - Comparison of different statistical characteristics:

- Mean value, minimum/maximum values, standard deviation, and histograms

3. Control Performance Analysis - NERC CPS2 index.

\subsection{Validation Results}

\subsubsection{Visual Inspection}

Figure 3.1 to Figure 3.7 show the comparison among actual output of AGC units and simulated output of AGC units, as well as actual and simulated ACE time series. From the total AGC generation, individual AGC unit output, to the time series of ACE, simulation and actual data match approximately. The discrepancies are considered acceptable because real-time adjustments by operators on the AGC units were not included in the simulation.

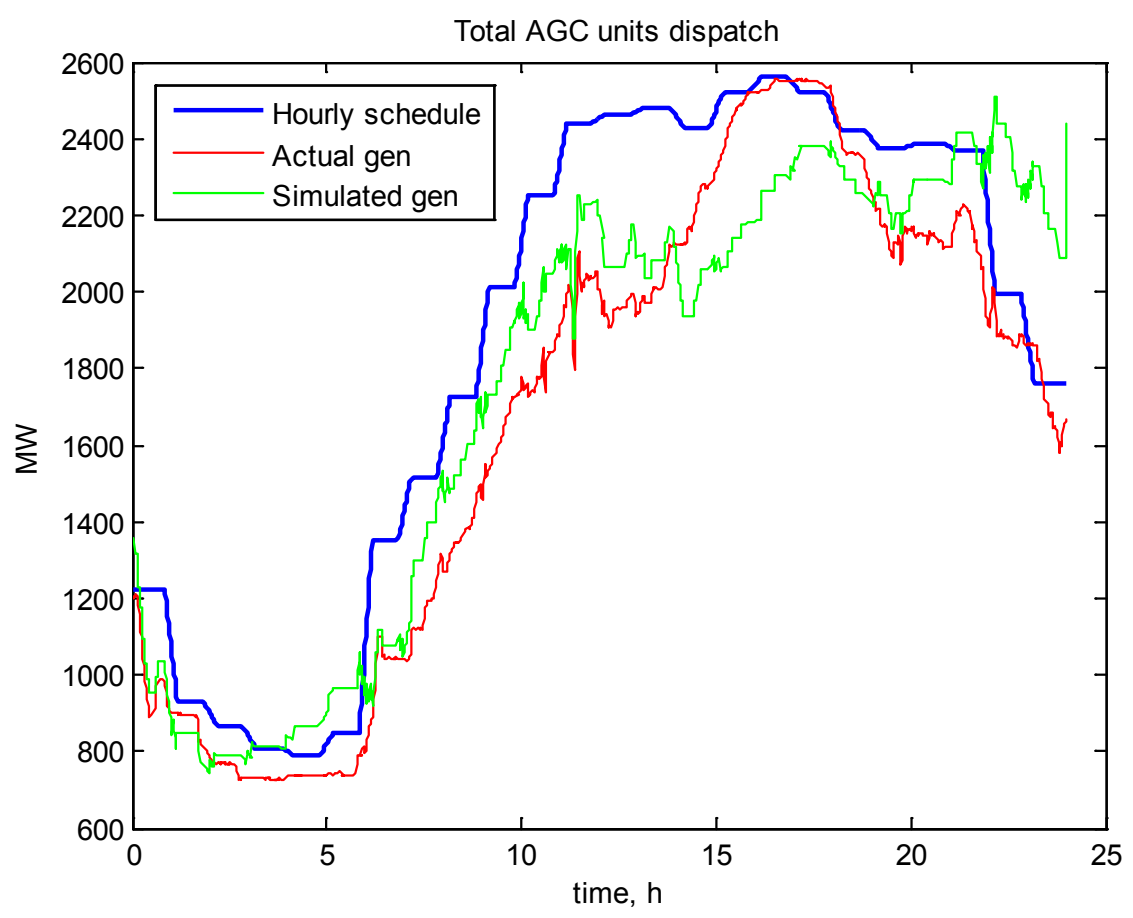

Figure 3.1. AGC Units Total Dispatch (August 1, 2012) 


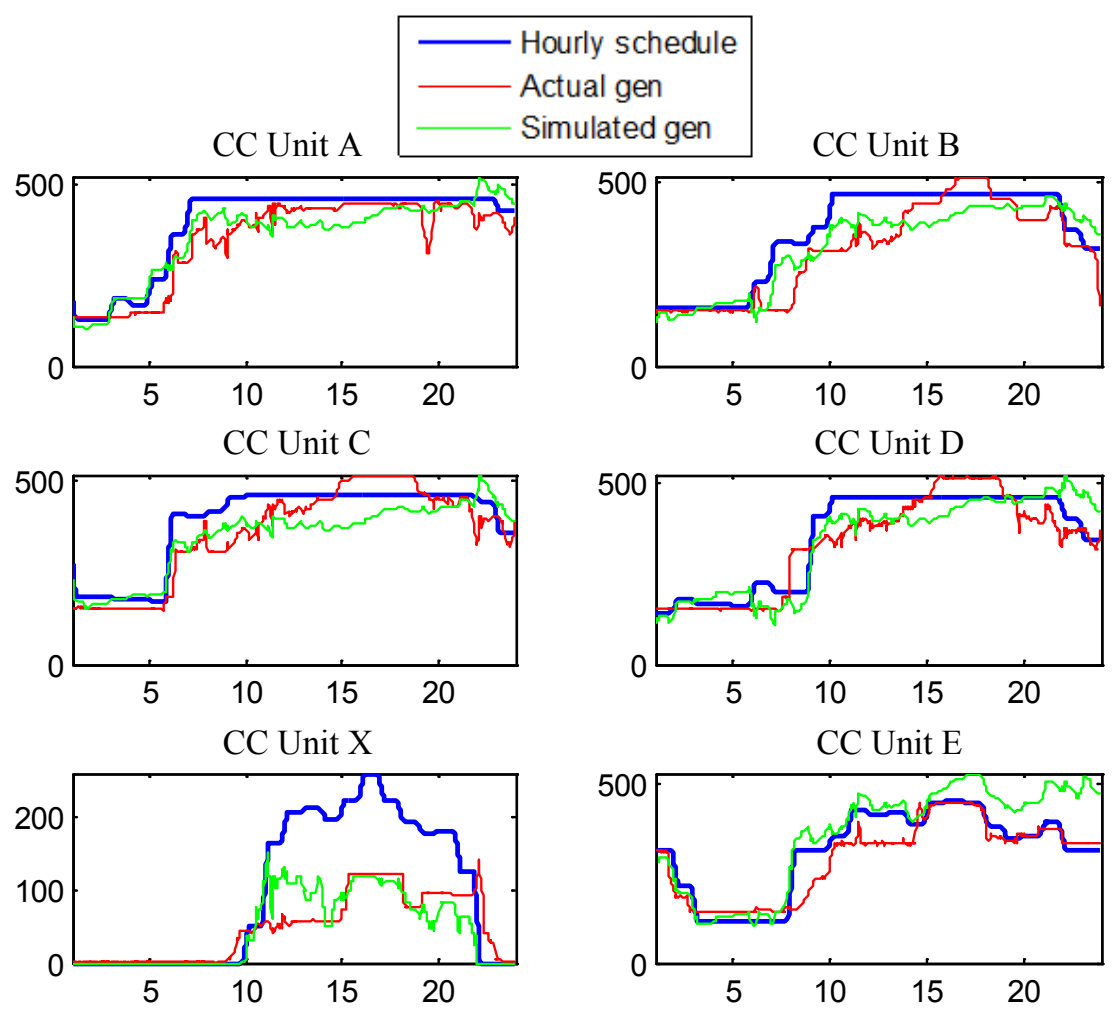

Figure 3.2. AGC Units Dispatch (August 1, 2012)

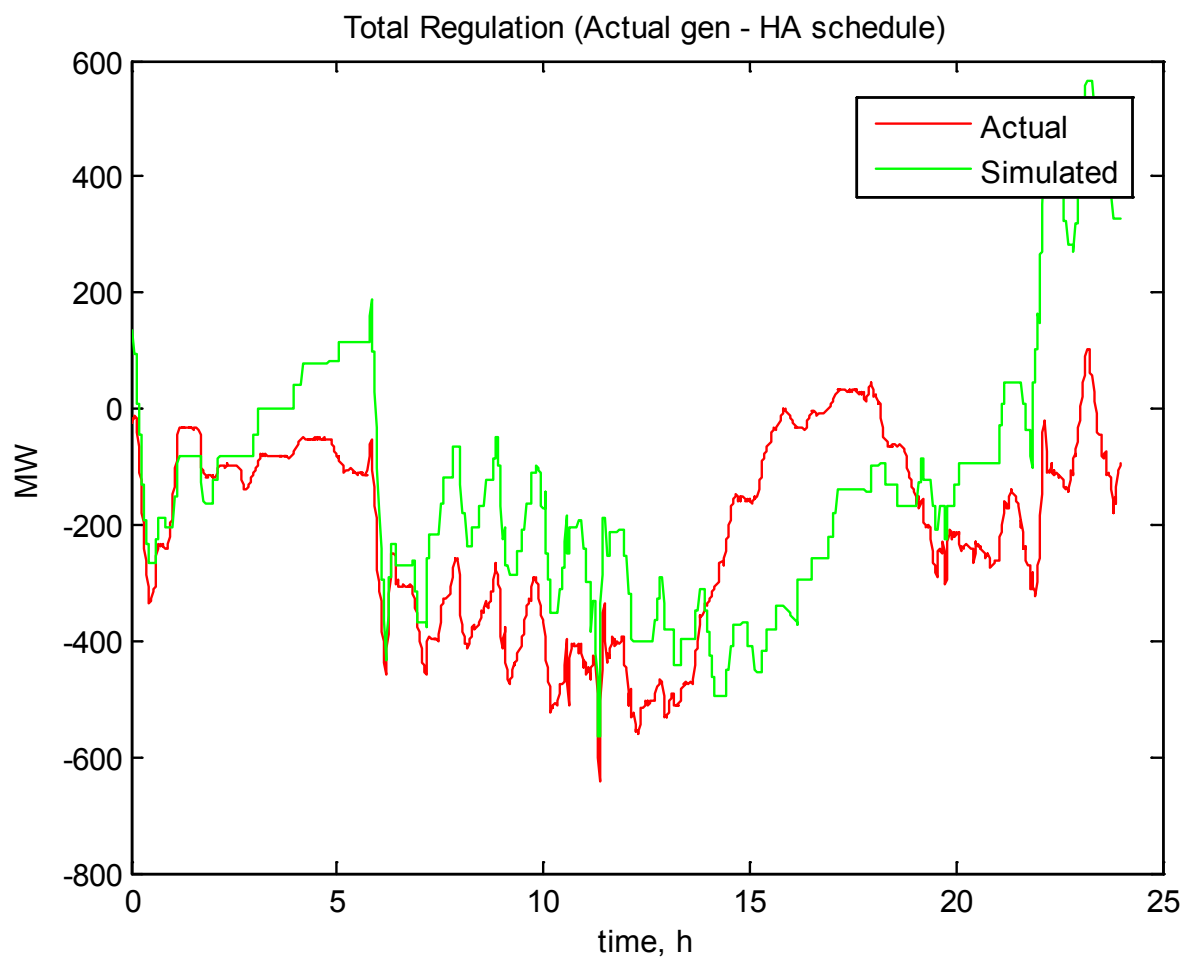

Figure 3.3. Total Regulation Applied (August 1, 2012) 


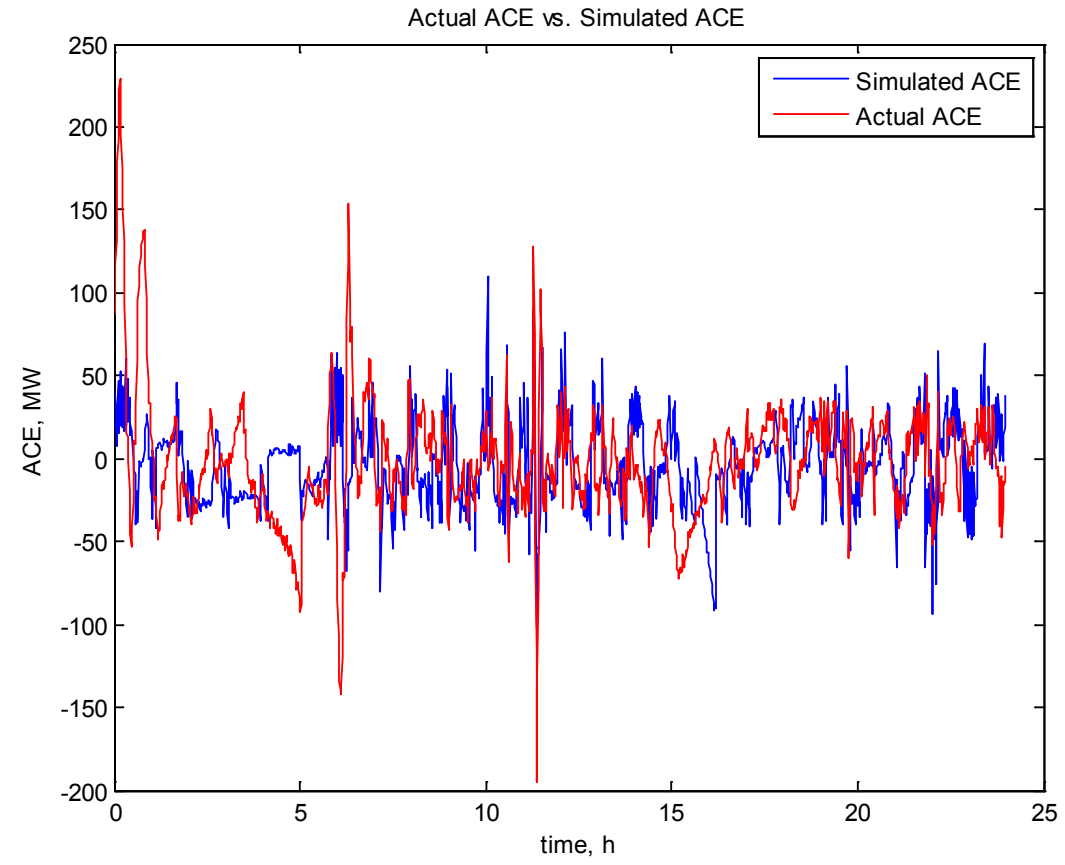

Figure 3.4. ACE Signal (August 1, 2012)

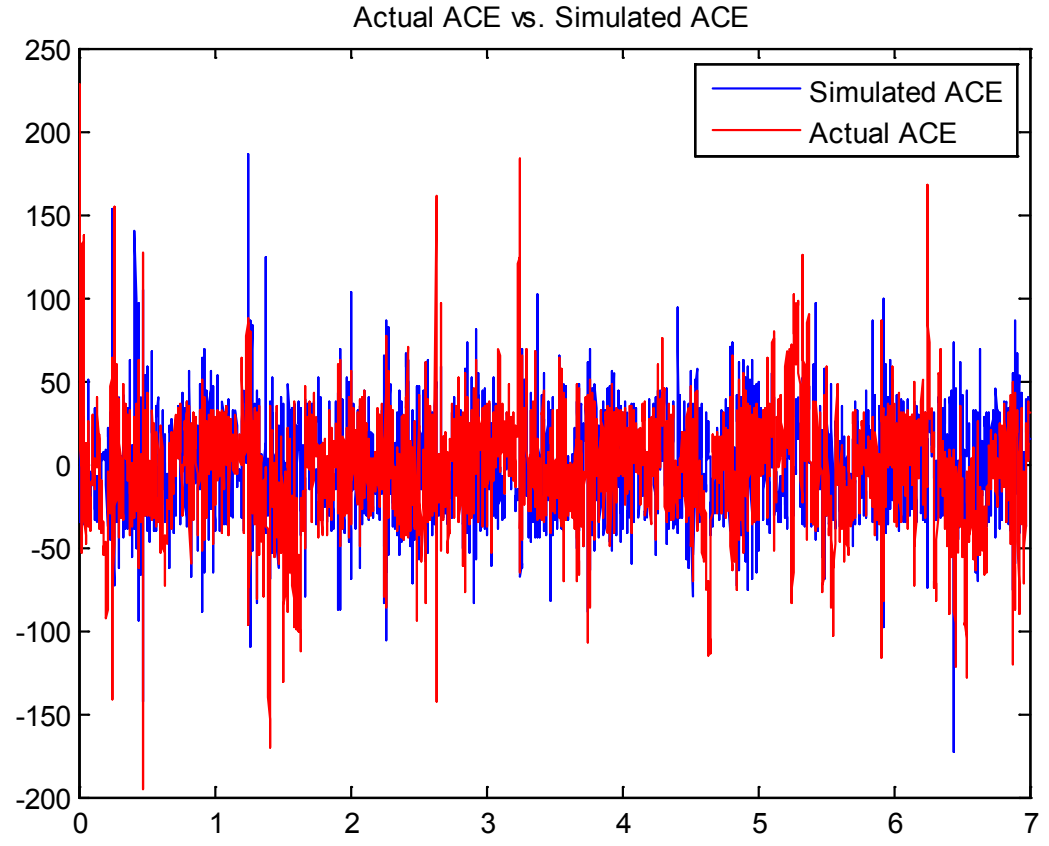

Figure 3.5. ACE Signal (August 1-7, 2012) 


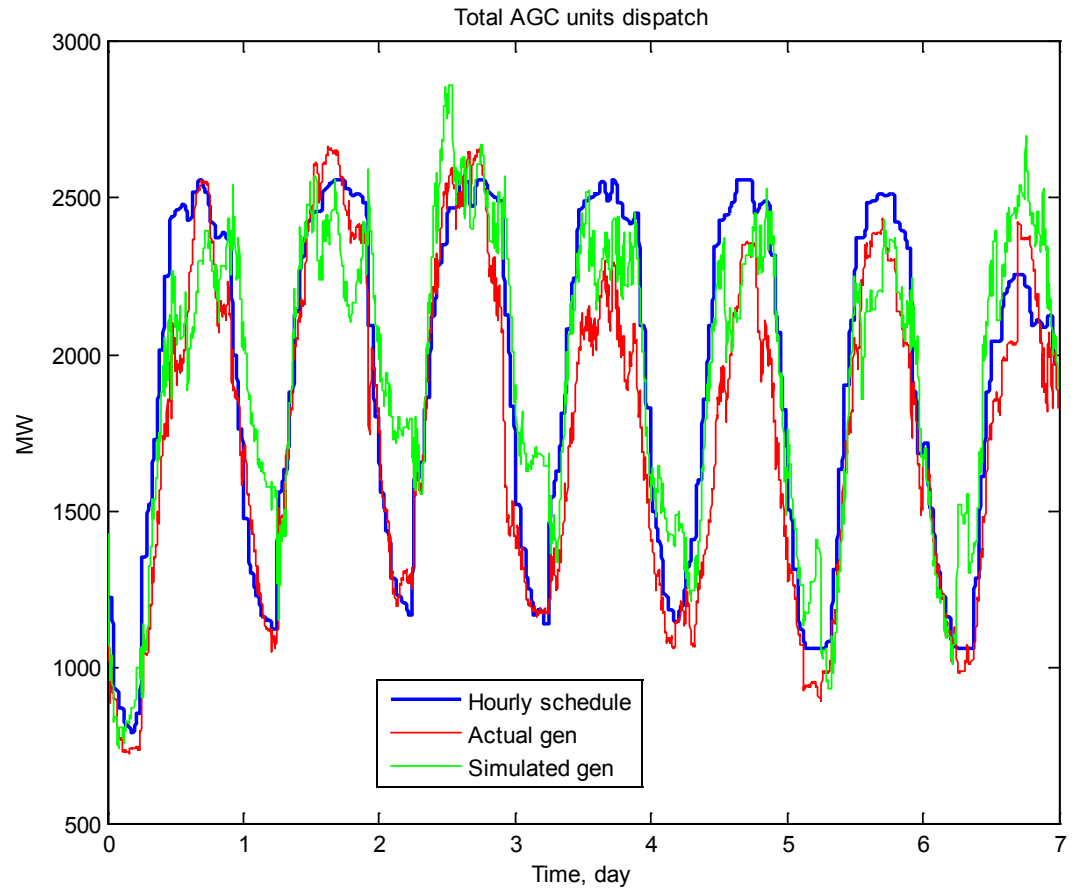

Figure 3.6. AGC Units Total Dispatch (August 1-7, 2012)
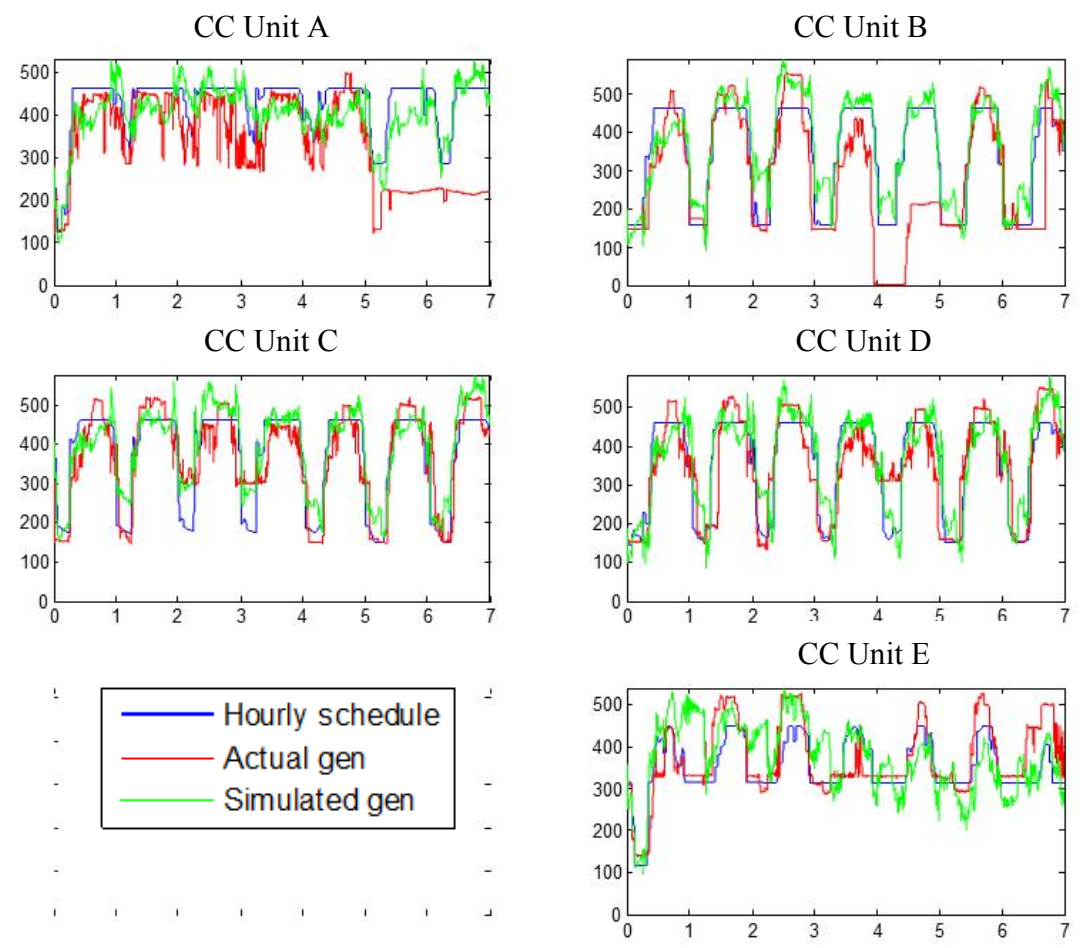

Figure 3.7. AGC Units Dispatch (August 1-7, 2012) 


\subsubsection{Statistical Analysis}

Figure 3.8 and Figure 3.9 compare the histogram of ACE for the entire validation period and each hour, respectively. Table 3.1 lists the statistical properties of the actual and simulated ACE for the validation period. A fairly good match can be observed between the simulation results and the actual data.

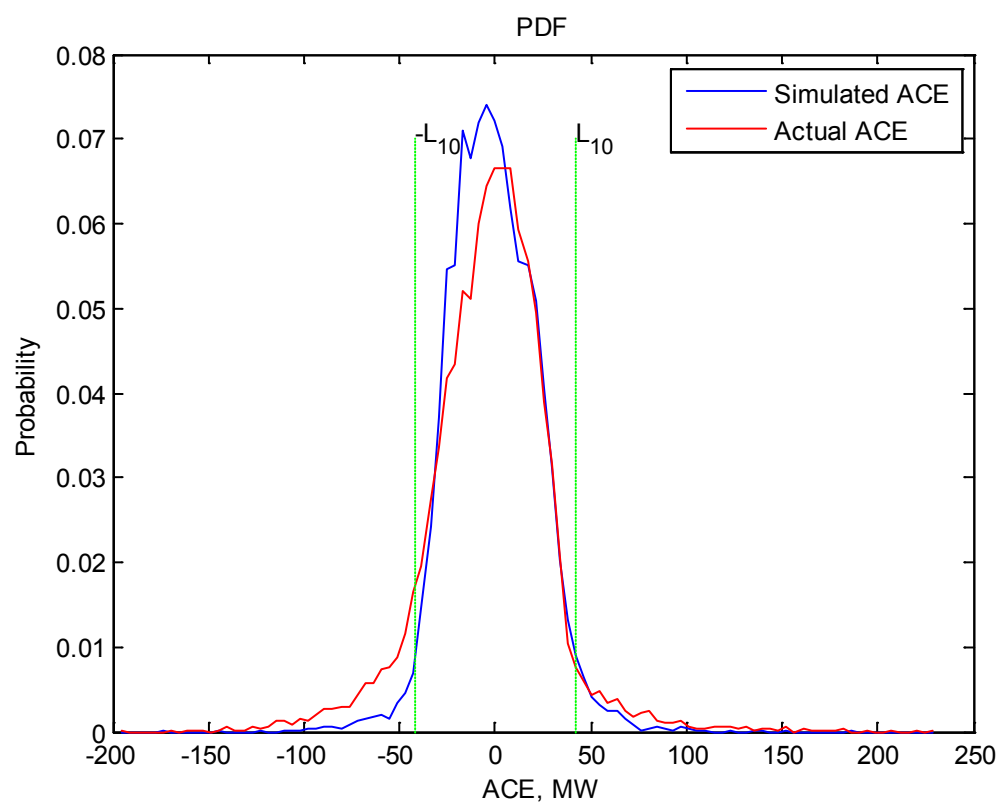

Figure 3.8. ACE Distribution (August 1-7, 2012)
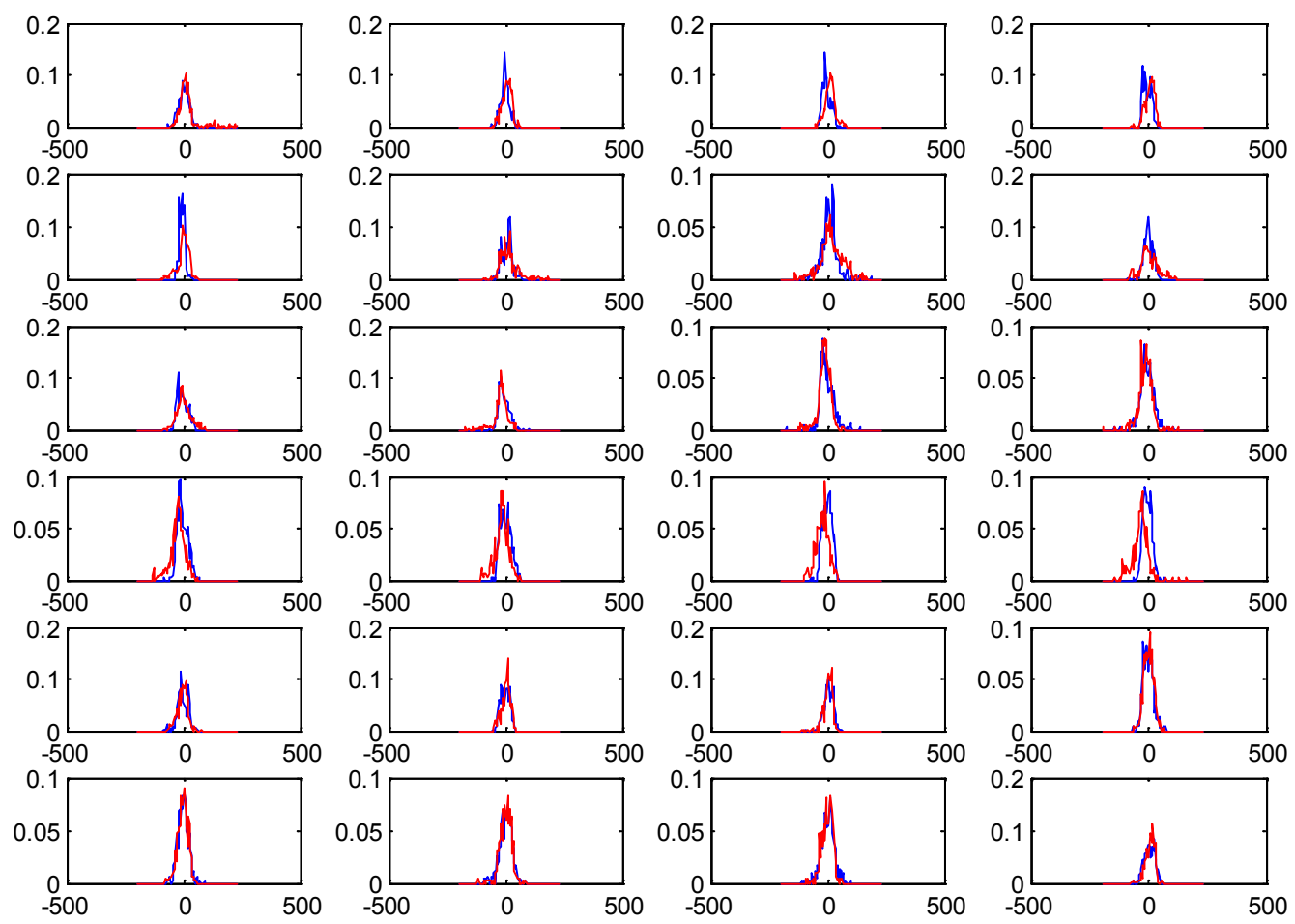

Figure 3.9. ACE Hourly Distribution (August 1-7, 2012) 
Table 3.1. ACE Statistical Characteristics

\begin{tabular}{ccc}
\hline & Simulated ACE & Actual ACE \\
\hline Mean & -1.19218 & -2.76212 \\
STD & 23.19262 & 32.18171 \\
Min & -172.854 & -195.111 \\
Max & 186.4068 & 228.6277 \\
\hline
\end{tabular}

\subsubsection{Control Performance Analysis}

Table 3.2 lists the CPS2 performance index under different AGC parameter settings. It demonstrates that different level of CPS2 compliance can be achieved by adjusting AGC parameters.

Table 3.2. CPS2 Index

\begin{tabular}{ll}
\hline & CPS2 \\
\hline Actual & $90.2 \%$ \\
Simulated $\left(\mathrm{a}=1.0, \mathrm{~K} \_\mathrm{R}=\mathrm{K} \_\mathrm{A}=1.0, \mathrm{REGDB}=\mathrm{EADB}=30 \mathrm{MW}\right)$ & $99.5 \%$ \\
Simulated $\left(\mathrm{a}=1.0, \mathrm{~K} \_\mathrm{R}=\mathrm{K} \_\mathrm{A}=0.8, \mathrm{REGDB}=\mathrm{EADB}=30 \mathrm{MW}\right)$ & $97.3 \%$ \\
Simulated $\left(\mathrm{a}=1.0, \mathrm{~K} \_\mathrm{R}=\mathrm{K} \_\mathrm{A}=0.8, \mathrm{REGDB}=\mathrm{EADB}=45 \mathrm{MW}\right)$ & $95.1 \%$ \\
Simulated $\left(\mathrm{a}=1.0, \mathrm{~K} \_\mathrm{R}=\mathrm{K} \_\mathrm{A}=0.6, \mathrm{REGDB}=\mathrm{EADB}=45 \mathrm{MW}\right)$ & $91.1 \%$ \\
\hline
\end{tabular}

\subsubsection{Results Summary of Simulator Validation}

Through comparison between the simulated AGC process and actual data, one can see that the simulation could approximately match what really happened in the system, in terms of both AGC generator outputs and the distribution of ACE. Considering the fact that AGC parameters can be adjusted to change the level of control performance, and the simulation did not include dispatch by operators that happened in real time, the differences between simulation results and the actual data are deemed acceptable. As discussed in Section 2.2.1, the AGC simulator should be adequate for use in the investigation of generation fleet flexibility and control performance with various amounts of PV generation. 



\subsection{Simulation of PV Integration Cases}

\subsection{Assumptions and PV Cases}

The main objective of this part of work is to evaluate the actual deployment of AGC and peaking units and assess system control performance at various PV penetration levels. The impact of PV on system intra-hour operations balancing generation and load can be better examined through such detailed system simulations.

The unit commitment process, which determines hourly generation schedules, is produced using Ventyx PROMOD software tool ${ }^{1}$ (any other unit commitment tool would work as well). The hourly schedules of generators are input to ESIOS program, based on which ESIOS produces the intra-hour dispatch and AGC results. Similar to the validation process and with some differences, the simulations were conducted with the following assumptions:

- Load and PV forecast errors are not considered. ${ }^{2}$ In other words, perfect forecasts are assumed because of the lack of forecast data.

- Economic dispatch is performed every minute in AGC. No separate real-time dispatch (or load following) process is simulated.

- AGC Units (units used for regulation) include CC Units A through E.

- Non-AGC units follow exactly their hourly schedules, and 20-minute ramps are used between the schedules of two neighboring hours.

- Net Interchange exactly equals the hourly schedules and follows the 20-minute ramps between adjacent hours.

- AGC units can perform regulation within $\mathrm{Reg}_{\min }$ to $\mathrm{Reg}_{\max }$ corresponding to each configuration, as defined in the characteristics tables.

- $\mathrm{L}_{10}$ of the NV Energy southern system is $42.23 \mathrm{MW}$.

- There are no contingencies in the system.

Generator heat-rate curves are used to performed economic dispatch with respect to their ramp rate constraints. ACE is calculated as the system imbalance between generation and load with consideration of interchanges.

The same data set was used in the simulations as in the 2011 NV Energy southern system PV integration study, which includes: actual system load in 2007, solar generation (simulated for different penetration cases under weather conditions of 2007), interchange, and generation characteristics.

\footnotetext{
${ }^{1} \mathrm{http} / / /$ www.ventyx.com/en/enterprise/business-operations/business-products/promod-iv

${ }^{2}$ It is important to note that forecast uncertainties were not simulated in this work because of the lack of data. The balancing operations simulated in the results are, therefore, mainly dealing with the inherent variability of load and $\mathrm{PV}$ generation. It is anticipated that load and PV forecast uncertainties can significantly impact balancing reserve requirements in terms of both capacity and ramp rates. They should be analyzed when data are available.
} 
Table 4.1 lists the PV penetration cases that were studied in this effort. Case 1A to Case 5A each contains $50 \mathrm{MW}$ of distributed PV and $149 \mathrm{MW}$ to $892 \mathrm{MW}$ of large-scale PV generation. To put the amount of PV installation capacity into context, the monthly minute-to-minute load peaks and minimums are shown in Table 4.2.

Table 4.1. PV Study Cases

\begin{tabular}{ccc}
\hline & DG - Percent of Peak Load \\
\cline { 3 - 3 } PV/DG Cases & $1 \%(50 \mathrm{MW})$ \\
\hline Large PV & Case 1 - 149 MW & 1A \\
& Case 2-222 MW & $2 \mathrm{~A}$ \\
Case 3 - 292 MW & $3 \mathrm{~A}$ \\
Case 4 - 492 MW & $4 \mathrm{~A}$ \\
Case 5-892 MW & $5 \mathrm{~A}$ \\
\hline
\end{tabular}

Table 4.2. NV Energy Southern System Load Peaks by Month

\begin{tabular}{ccccccccccccc}
\hline Month & Jan & Feb & Mar & Apr & May & Jun & Jul & Aug & Sep & Oct & Nov & Dec \\
\hline $\begin{array}{c}\text { High } \\
\text { (MW) }\end{array}$ & 2827 & 2489 & 2773 & 3634 & 4389 & 5174 & 5912 & 5438 & 5118 & 3172 & 2712 & 2760 \\
$\begin{array}{c}\text { Low } \\
\text { (MW) }\end{array}$ & 1800 & 1657 & 1535 & 1501 & 1478 & 1699 & 2161 & 2131 & 1559 & 1545 & 1549 & 1720 \\
\hline
\end{tabular}

\subsection{Example Results of January in Case 1A and Case 5A}

This section presents the simulation results of January in Case 1A and Case 5A among the NV Energy PV study cases. Plots including total and individual AGC unit schedules and dispatch, CC unit configurations, ACE, available spinning and regulation reserves, and status of peaking units are shown in Sections 4.2.1 and 4.2.2 for each case. These examples illustrate the information provided by ESIOS and also give an opportunity for readers to compare the two extreme cases. Results of a few selected days are analyzed closely to verify that the simulator replicates AGC and operator functions in the way it is designed. Section 4.2.3 describes the deployed AGC reserves and disaggregates them into regulation (fast or minute-by-minute) and load-following (slower or 10-minute) components to compare with the corresponding requirements developed in the 2011 PV integration study [1]. Distributions of ACE and control performance index CPS2 are compared in Section 4.2.4 between Case 1A and Case 5A. Results from all simulated cases are summarized next in Section 4.3.

\subsubsection{AGC and Peaking Units Dispatch in Case 1A}

Figure 4.1 shows the total minute-by-minute output of AGC and peaking units during January in Case $1 \mathrm{~A}$, as well as the ACE through the month. The output very closely follows the load demand on these units (total net load deducted by the part undertaken by non-AGC and non-peaking units) most of the time. Figure 4.2 compares the total AGC unit output to the total scheduled load on these units. Because accurate load and PV generation data (no forecast errors) were used when producing the unit commitment and hourly scheduling results, the schedule and actual dispatch of AGC units are quite close. Figure 4.3 compares the actual dispatch and schedule of each individual AGC unit. 
Figure 4.4 shows the configuration of each AGC unit over the entire month. All of the five AGC units simulated in the NV Energy southern system are natural gas CC units with two combustion turbines and one heat recovery steam turbine. When needed, each of the CTs can fire its DB to increase generation. Four different configurations or operation modes of $\mathrm{CC}$ units are modeled, which are labeled as $1 \times 1,2 \times 1,2 \times 1+\mathrm{DB}$, and $2 \times 1+2 \mathrm{DB}$ in Figure 4.4. Based on data from NV Energy, most CC units can provide regulation, in other words, be automatically dispatched by the AGC model, under $1 \times 1$ and 2 $\times 1$ modes $^{1}$. CC Unit E can only do so under $2 \times 1$ mode $^{2}$. When these units are committed in nonregulation modes, the ESIOS operator model can only adjust them to mimic the "manual" dispatch of operators. In Figure 4.3, the lower output of CC Unit E than its schedule on January 5 is an example of such dispatch actions.

Figure 4.5 and Figure 4.6 show the spinning and regulation reserves available in the system, respectively. The spinning reserve requirement was calculated dynamically based on the largest online generator output and other relevant data, as detailed in Section 2.4.1. In the NV Energy southern system, most spinning reserves are carried by the AGC generators; therefore, the total spinning reserve and RegUp reserve can be very close sometimes. Regulation reserve and AGC reserve are used interchangeably in the report (except in Section 4.2.3) to refer to the adjustable range (upward and downward from the operation point) of AGC units. When calculating regulation reserves, we assume the $\mathrm{CC}$ units do not follow the AGC signal during the transition period between two different configurations. This causes the system total regulation reserves to be zero if all online AGC units are changing operation modes simultaneously, as observed in some cases.

Figure 4.7 shows the starts and runtime of peaking units commanded by the operator model, in order to maintain sufficient spinning and regulation reserves, as discussed in Section 2.4.1. The total runtime of the peaking units for the entire month also are shown at the top of the plot. The runtime is calculated as generator-hours; for example, two peaking units operating for 1 hour would be counted as 2 generatorhours. The total runtime could be affected by the amount of PV generation, but could also be affected by the quality of the unit commitment and hourly scheduling results.

\footnotetext{
${ }^{1} \mathrm{CC}$ Units $\mathrm{C}$ and D currrently do not perform regulation in the $1 \mathrm{x} 1$ mode, but NV Energy is working to establish this capability.

${ }^{2}$ Currently, CC Unit E also can perform regulation under $1 \mathrm{x} 1$ mode.
} 

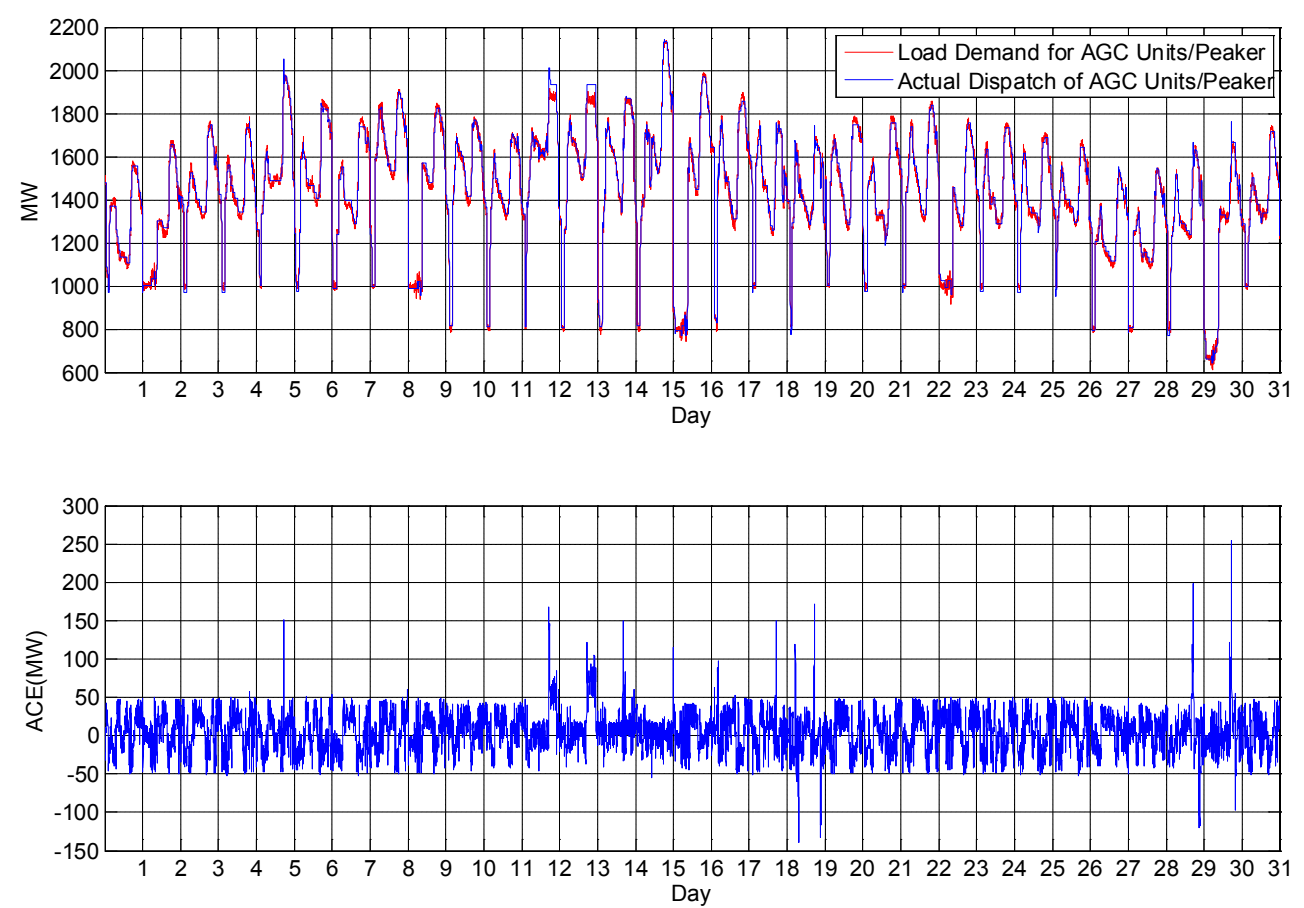

Figure 4.1. Dispatch of AGC and Peaking Units Compared to Load Demand on These Units (excluding the portion undertaken by generators following hour schedules) - January, Case 1A

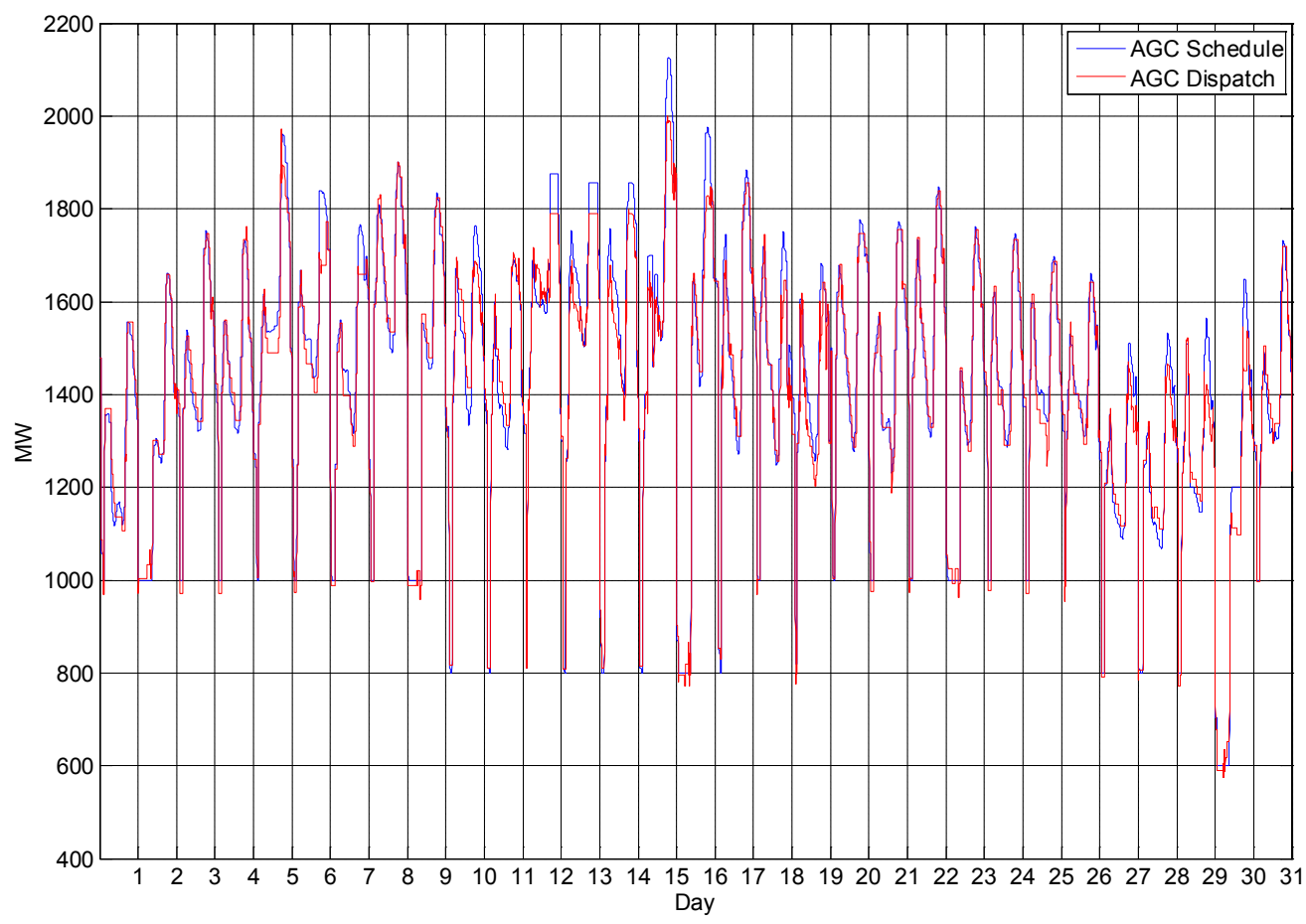

Figure 4.2. Total AGC Units Output Compared to their Hourly Schedule - January, Case 1A 

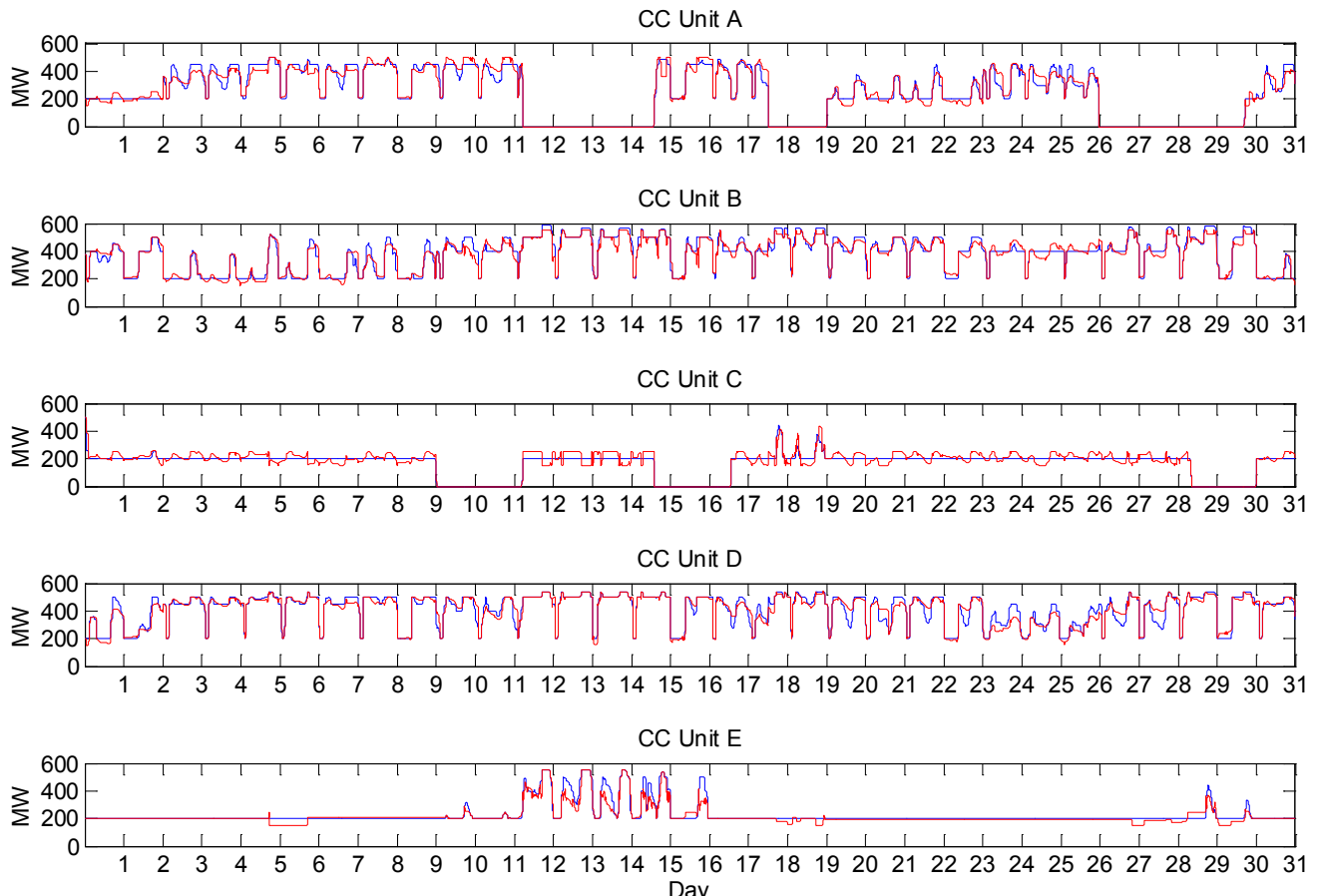

Figure 4.3. Dispatch of Each AGC Unit (red) Compared to Hourly Schedules (blue) - January, Case 1A
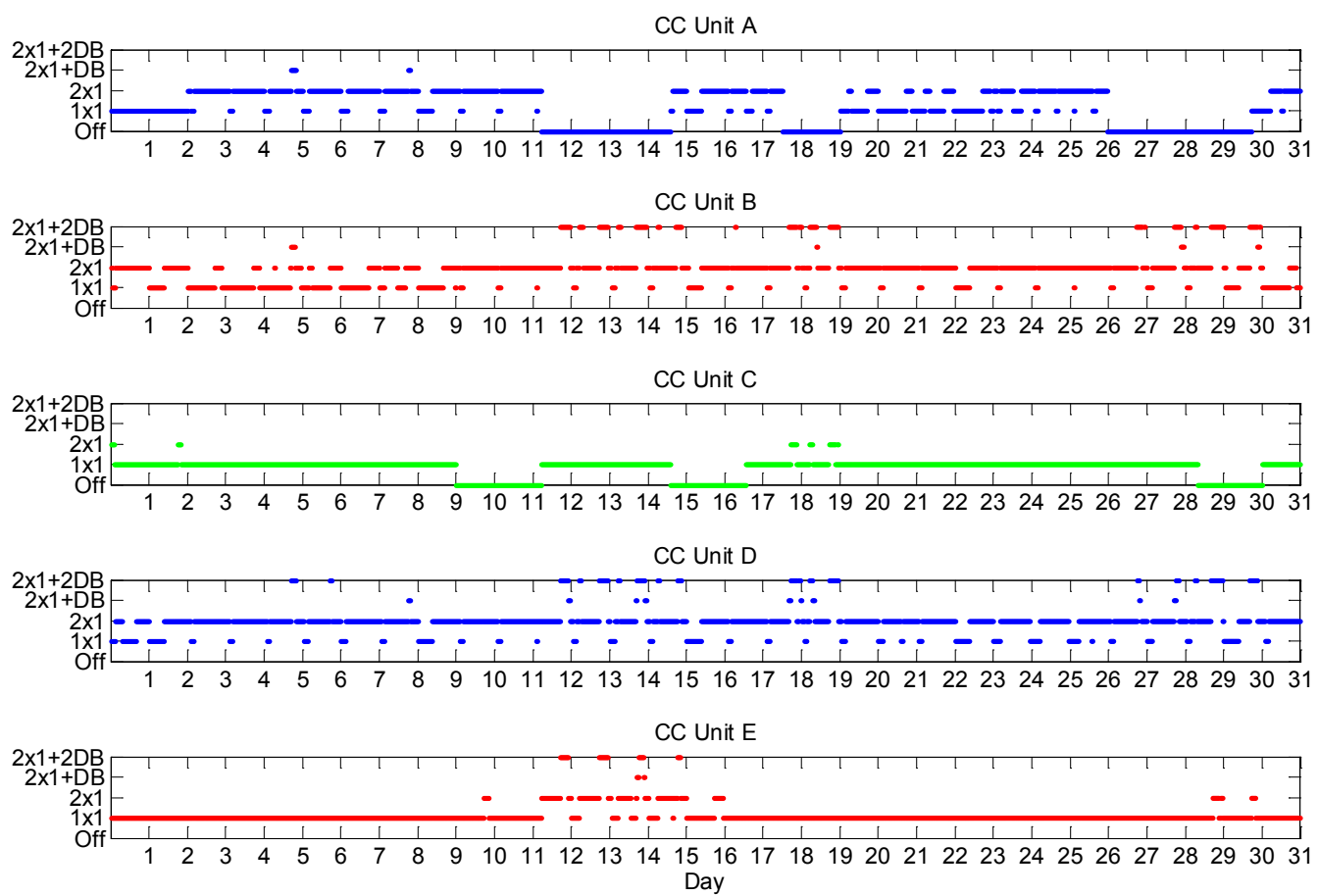

Figure 4.4. Configurations of AGC Units Determined by Unit Commitment - January, Case 1A 


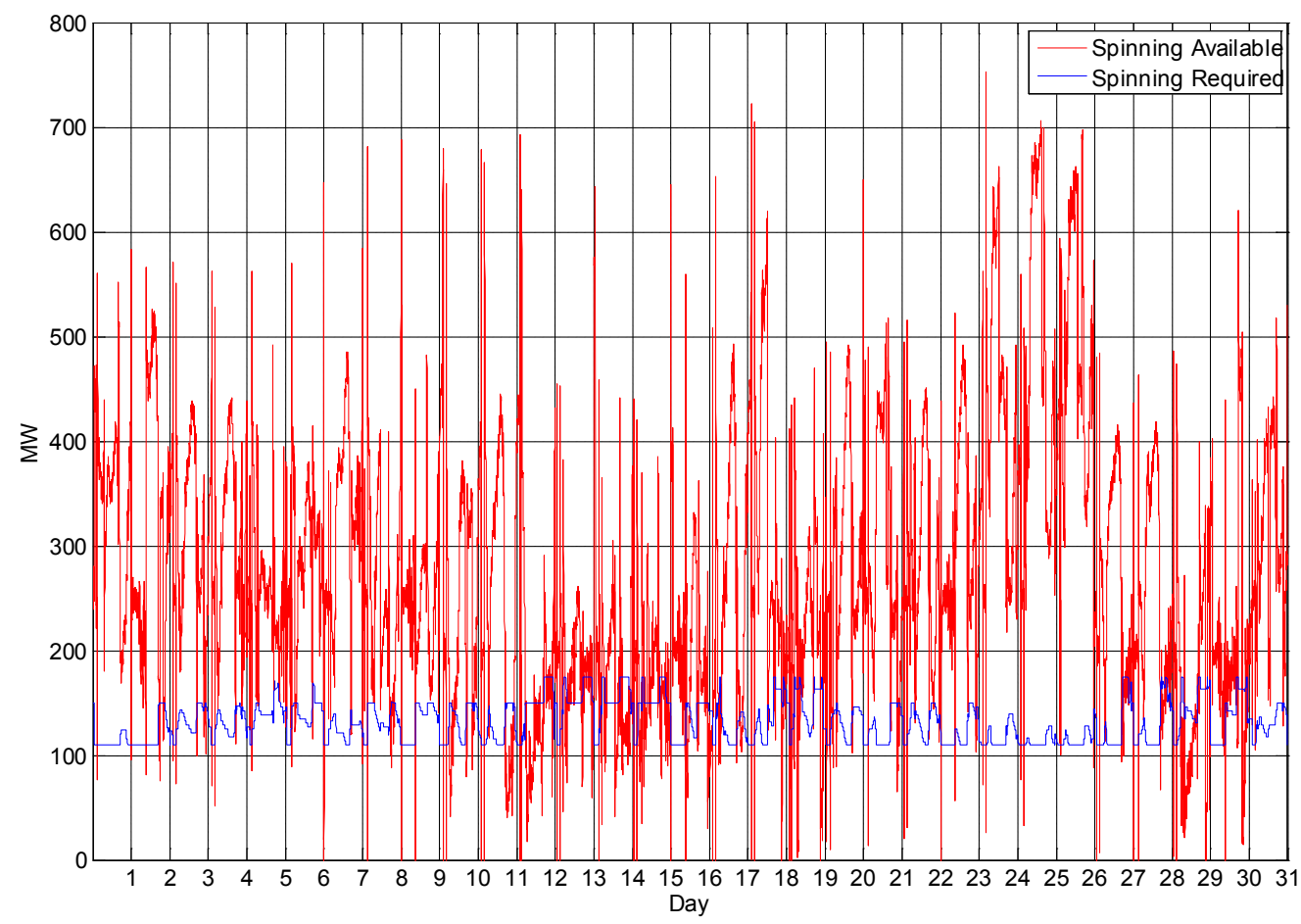

Figure 4.5. Available Spinning Reserve and Spinning Reserve Requirement - January, Case 1A

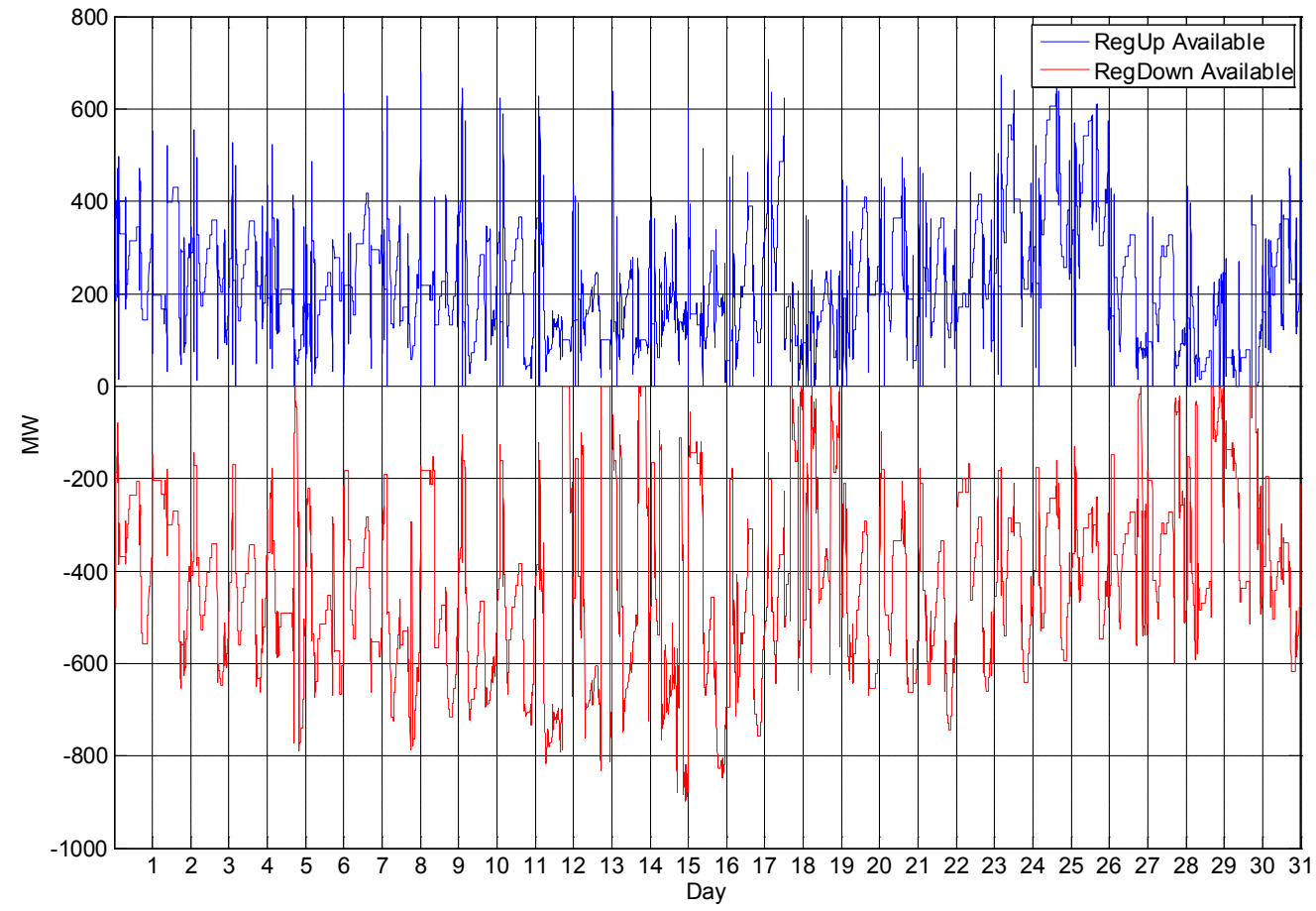

Figure 4.6. Available Regulation Reserves - January, Case 1A 


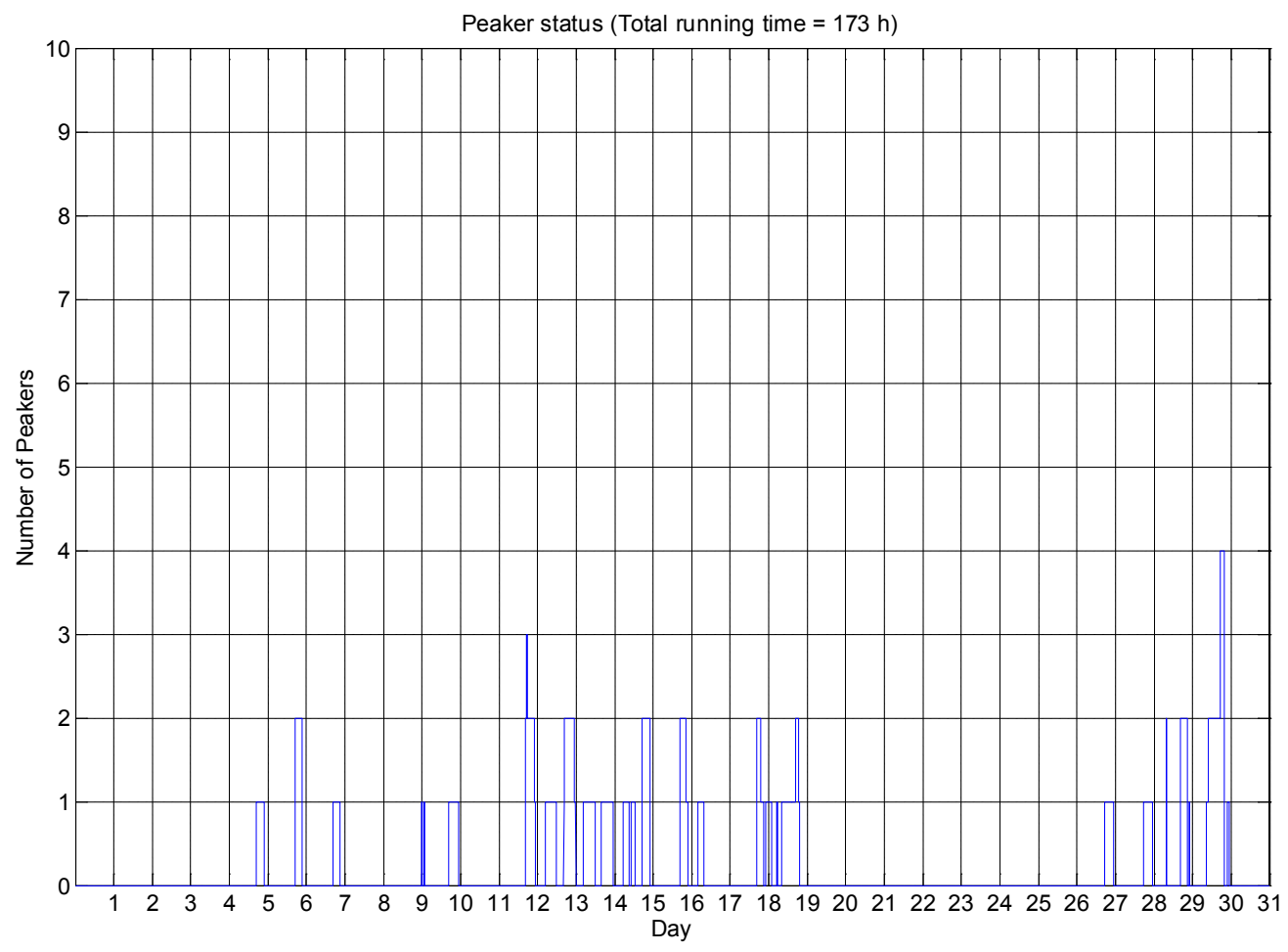

Figure 4.7. Peaking Units Dispatched by the Operator Model in ESIOS - January, Case 1A

Figure 4.8 presents a detailed look at the AGC and peaking units dispatch for the period from January 15 to January 17 .
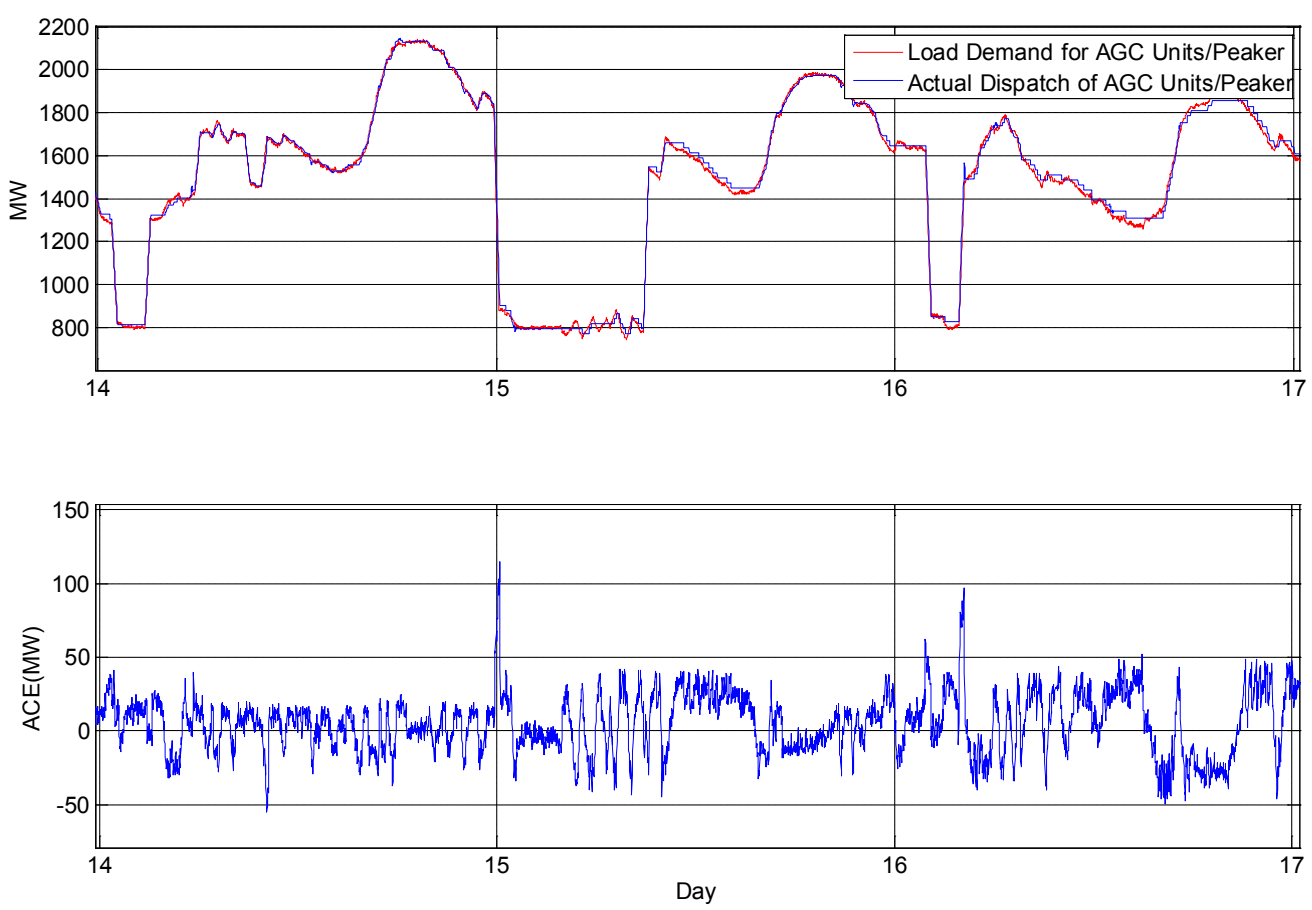

Figure 4.8. Dispatch of AGC and Peaking Units by ESIOS from January 15 to January 17 that have the Largest Daily Demand Cycles (difference between bottom and peak demand) of the Month 
The period in Figure 4.8 was selected for its largest daily demand cycles over the entire month, which could pose challenges to AGC generators when following the real-time load. ACE is seen to jump higher when there are steep ramps on AGC load. However, it is managed nicely most of the time.

Figure 4.9 plots the dispatch details of another period, from January 12 to January 14 . In both of the first two days, relatively large and positive ACE lasts for a few hours during the evening load peak. After some investigation, it was found that this was due to the lack of RegDn capability on the AGC units. Figure 4.10 shows the configuration of AGC units in these days. It can be seen that one AGC unit is offline, three units are scheduled in the $2 \times 1+2 \mathrm{DB}$ mode with no regulation ability, and the one AGC unit capable of regulation is already at its minimum output. Regulation reserve shortage during these large ACE periods can be further verified from Figure 4.11.

Although behavior of the simulator is reasonably explained, the above situation is considered resolvable in the real world. With sufficient generation online, some DBs of CC units could be turned off to reduce the positive $\mathrm{ACE}$ and provide room for regulation at the same time. However, in the simulator, because the operator model currently is not allowed to alter unit commitment results including the change of operation modes for CC units, the above solution was not simulated. This limitation renders the study a certain degree of conservativeness.
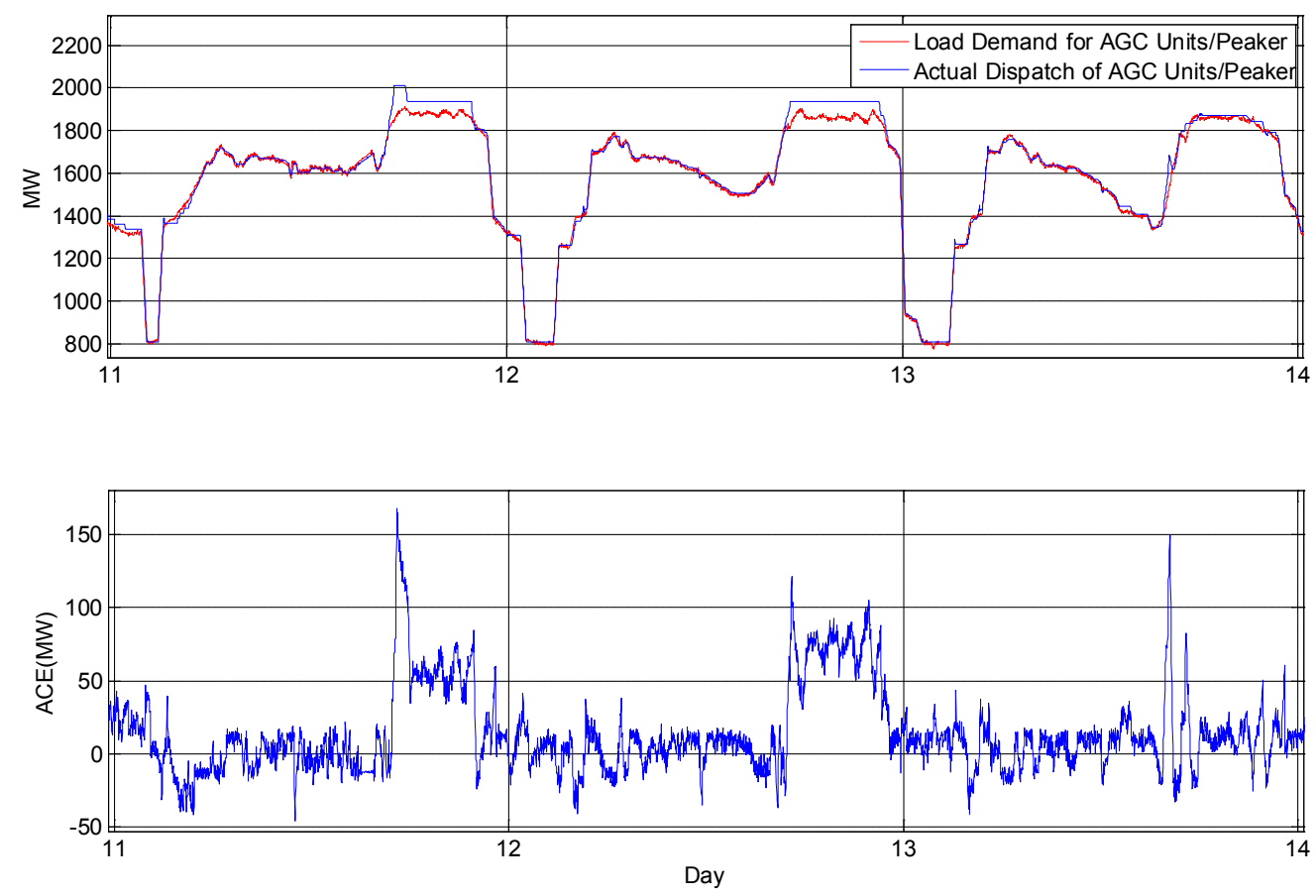

Figure 4.9. Dispatch of AGC and Peaking Units by ESIOS from January 12 to January 13 that have Large ACE Over a Prolonged Period 

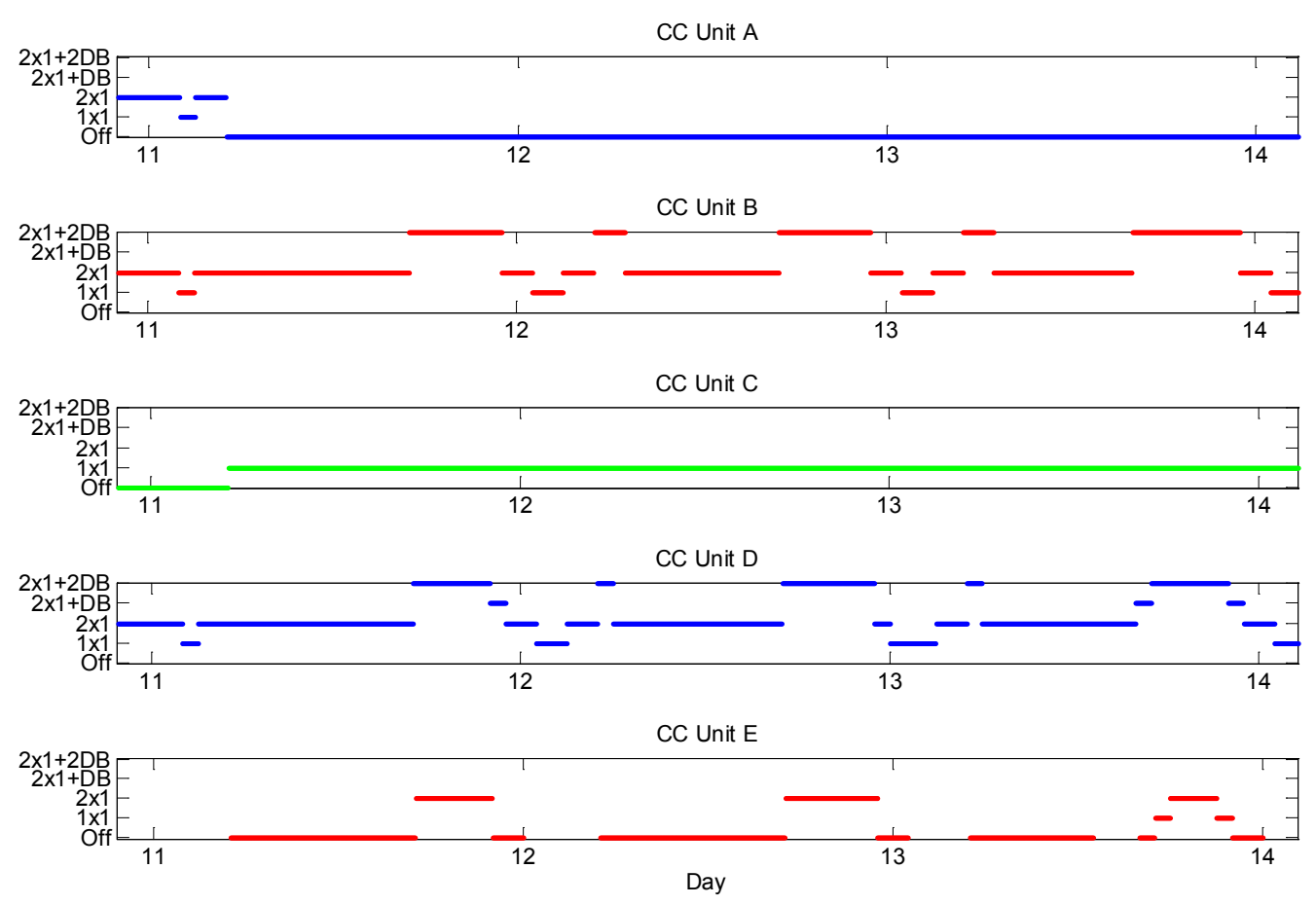

Figure 4.10. Configurations of AGC Units (determined by unit commitment) from January 12 to January 13 that have Large ACE Over a Prolonged Period

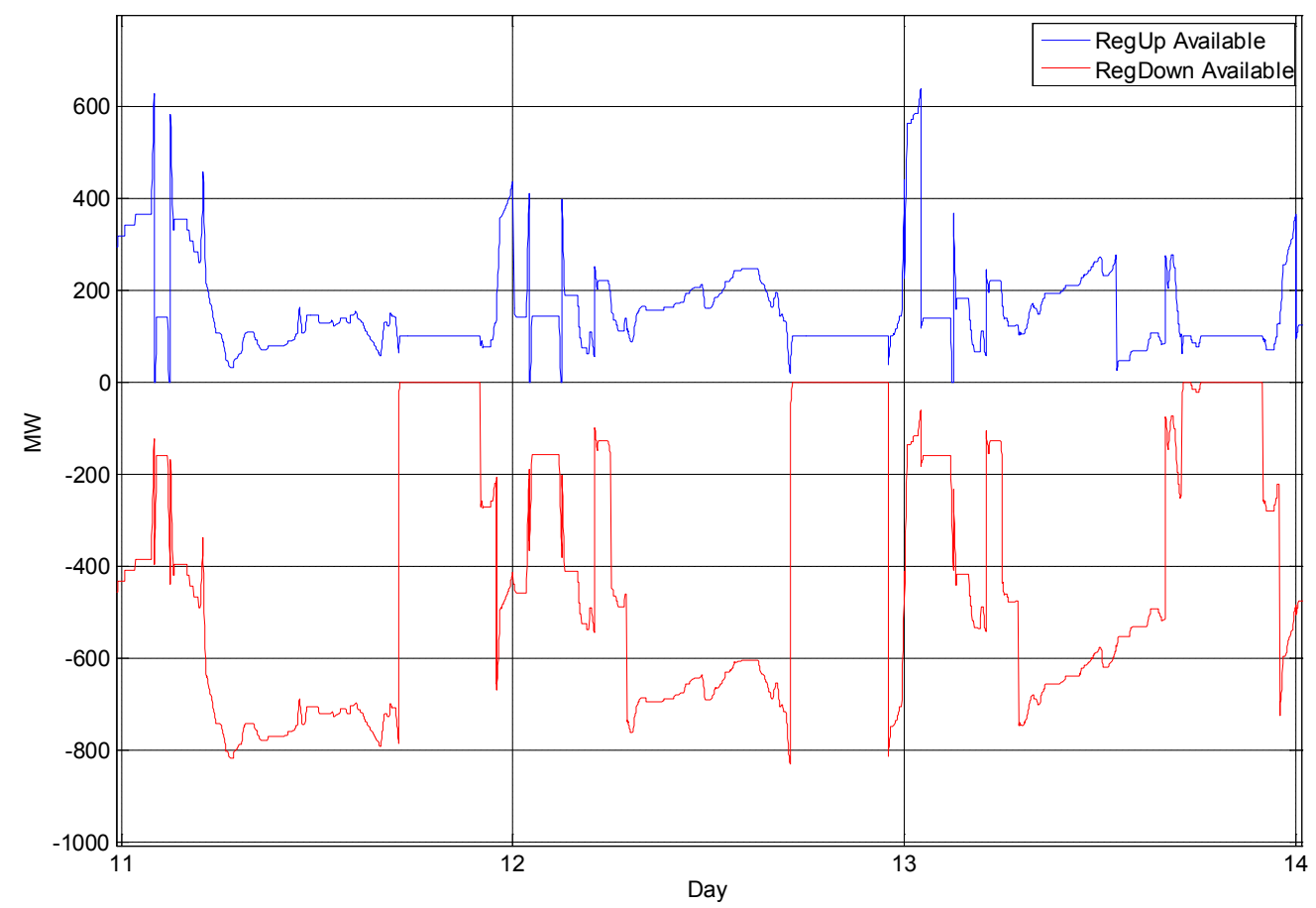

Figure 4.11. Available Regulation Reserves from January 12 to January 13 that have Large ACE over a Prolonged Period 


\subsubsection{AGC and Peaking Units Dispatch in Case 5A}

Figure 4.12 shows the actual dispatch of AGC and peaking units by ESIOS as compared to load demand on these units for January in Case 5A. The first couple of days see a larger than normal ACE for a few hours, which was investigated and is explained in Figure 4.13. Large differences are observed between the AGC units schedule and their load demand in the bottom plot. Because the actual load was used to produce the schedule, this discrepancy has to be attributed to the issue with the unit commitment software. The top plot in Figure 4.13 shows that the AGC units were dispatched far away from their schedule to try to meet the actual load demand, only limited by their operation range.

Figure 4.14 to Figure 4.19 present the comparison of output of AGC units and their schedule, CC unit configurations, available spinning and regulation reserves, and dispatch results of peaking units by the operator model, respectively.

These plots can be examined and compared to the corresponding results from Case 1A for the analysis of PV generation impacts. For example, for the period from January 20 to January 28 in Figure 4.12, the low peaks of demand were not matched by AGC generation, which were higher and flattened. This means the AGC generators have reached their minimum operation points and cannot be reduced further. Figure 4.18 does show that the regulation/AGC downward reserve is zero during these periods. The same problem is not observed in Case 1A in the corresponding plots (i.e., Figure 4.1 and Figure 4.6).
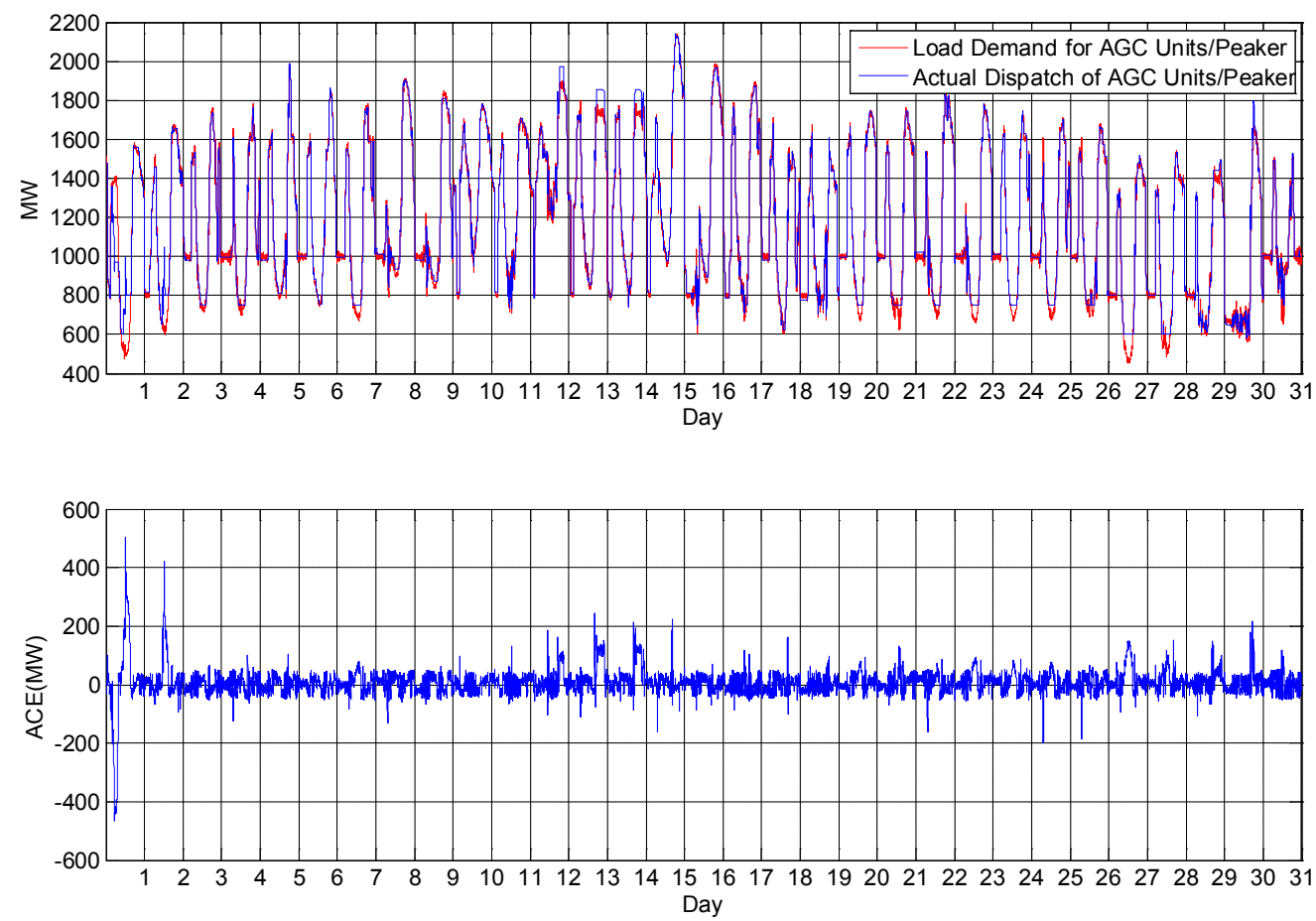

Figure 4.12. Dispatch of AGC and Peaking Units Compared to Load Demand on These Units (excluding the portion undertaken by generators following hour schedules) - January, Case 5A 

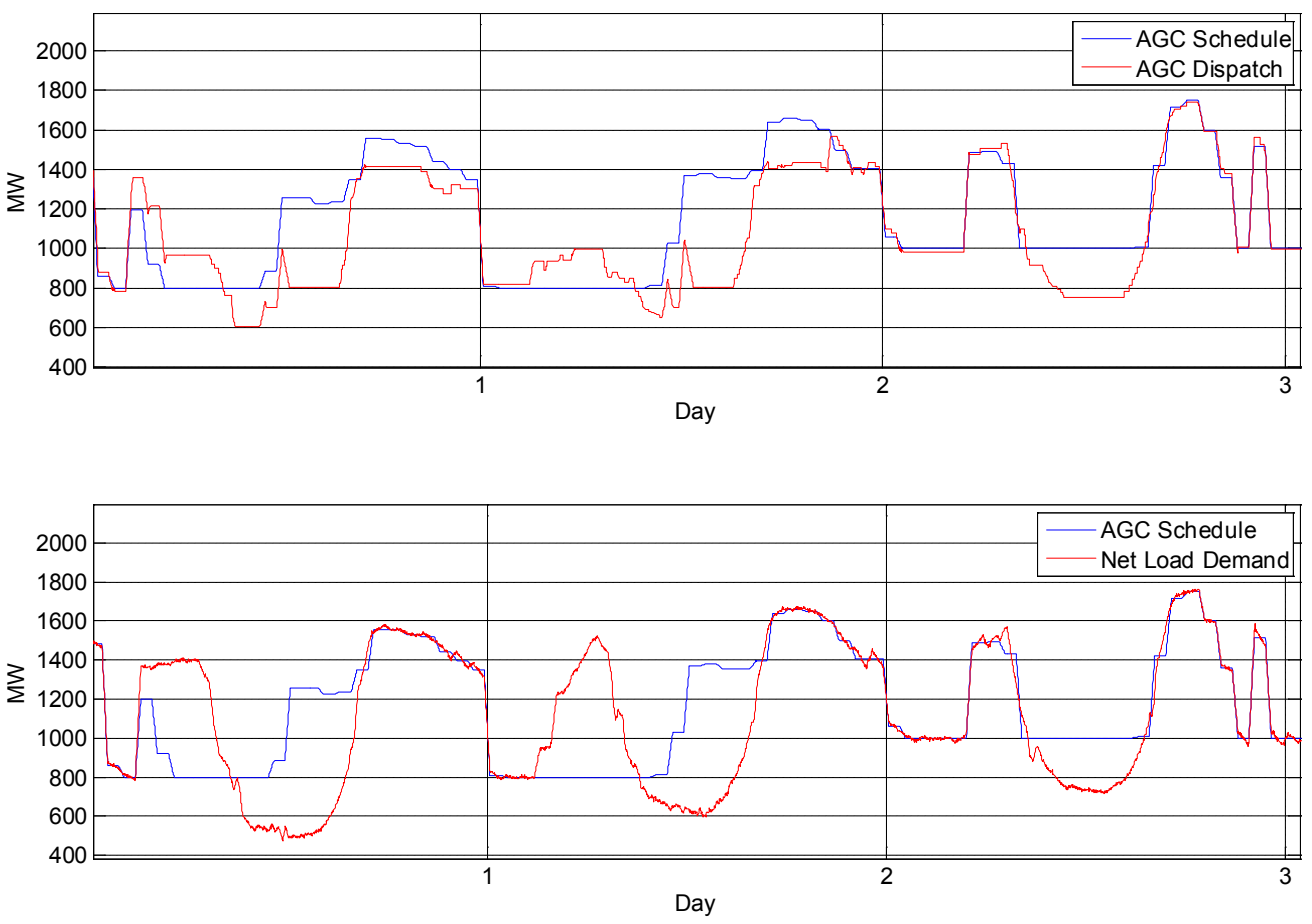

Figure 4.13. Dispatch of AGC Units from January 1 to January 3 when Significant Differences were Observed between the Schedule and Load Demand on AGC Units - Case 5A

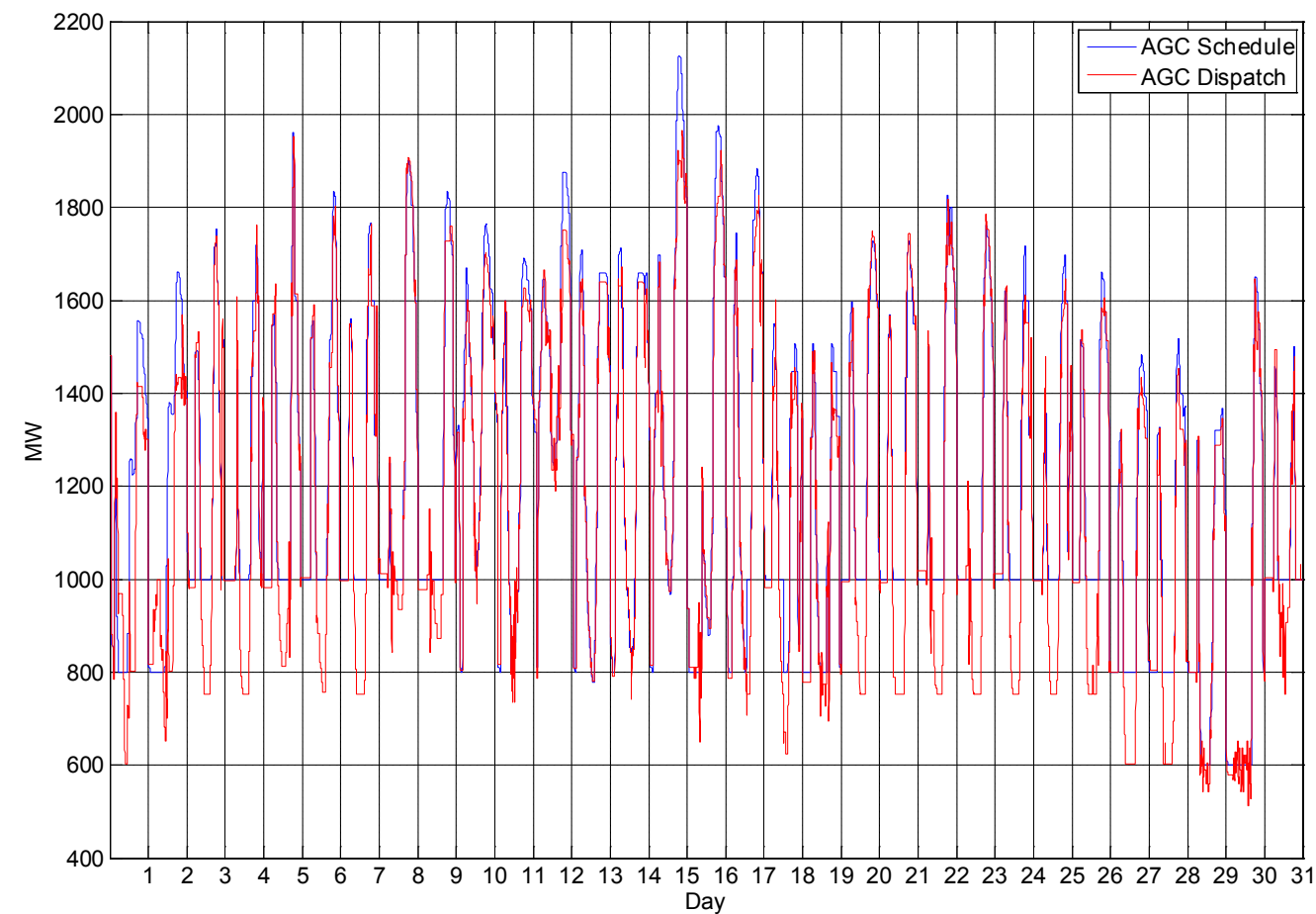

Figure 4.14. Total AGC Units Output Compared to their Hourly Schedule - January, Case 5A 

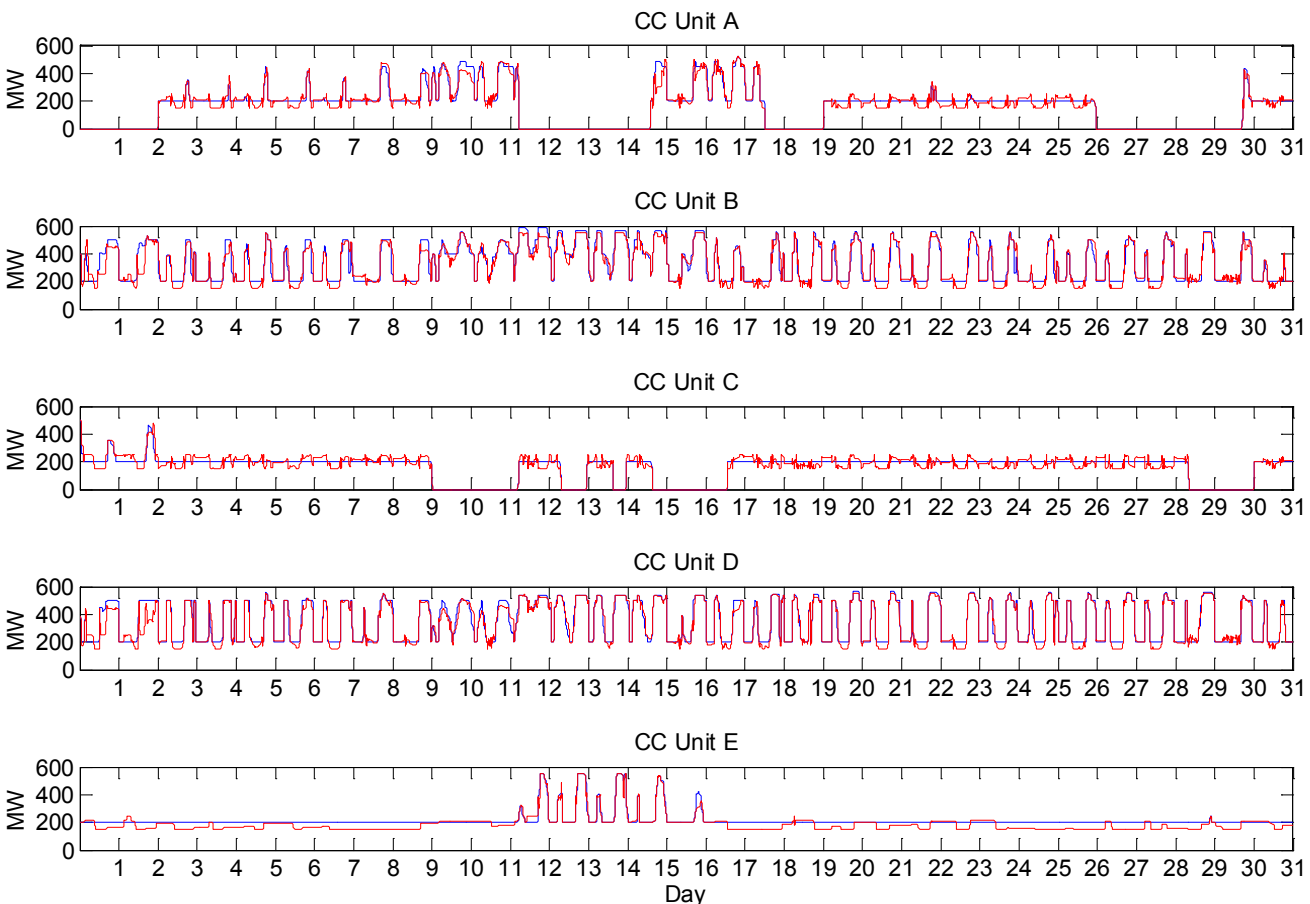

Figure 4.15. Dispatch of Each AGC Unit (red) Compared to Hourly Schedules (blue) - January, Case 5A
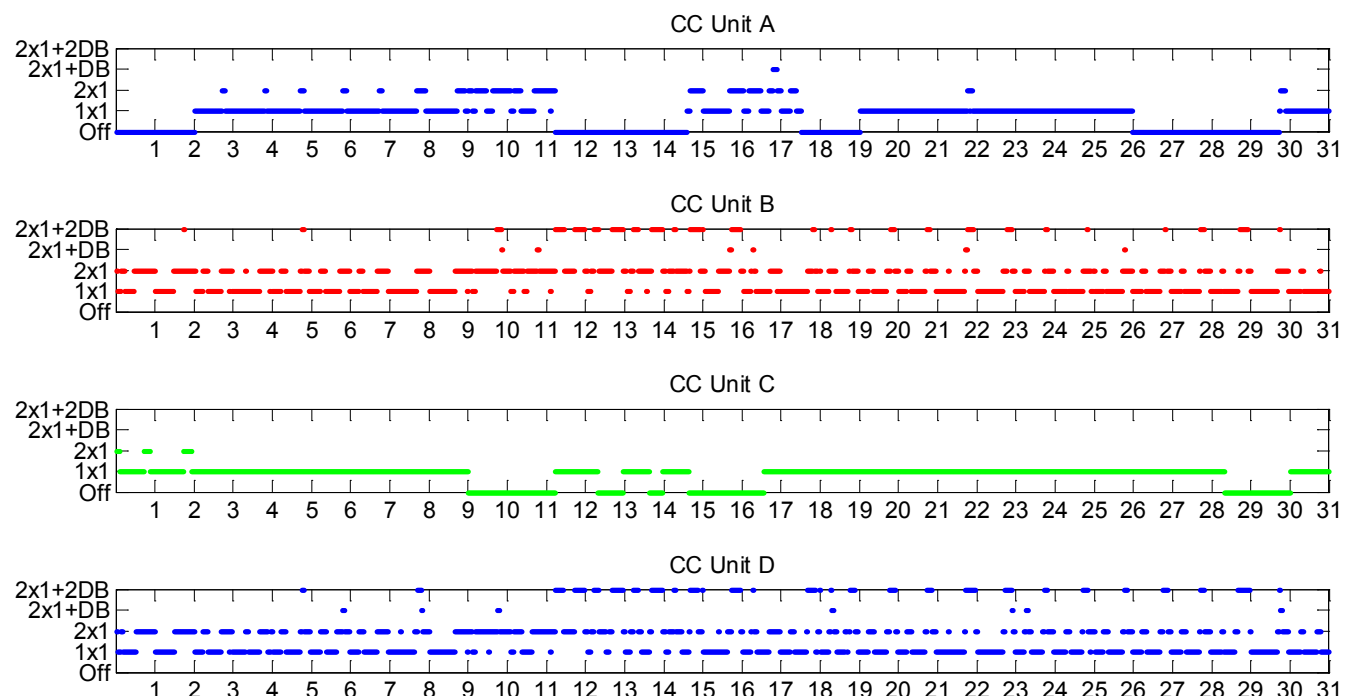

CC Unit E

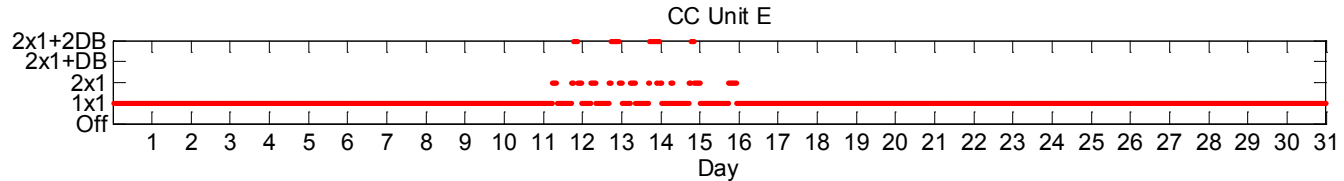

Figure 4.16. Configurations of AGC Units Determined by Unit Commitment - January, Case 5A 


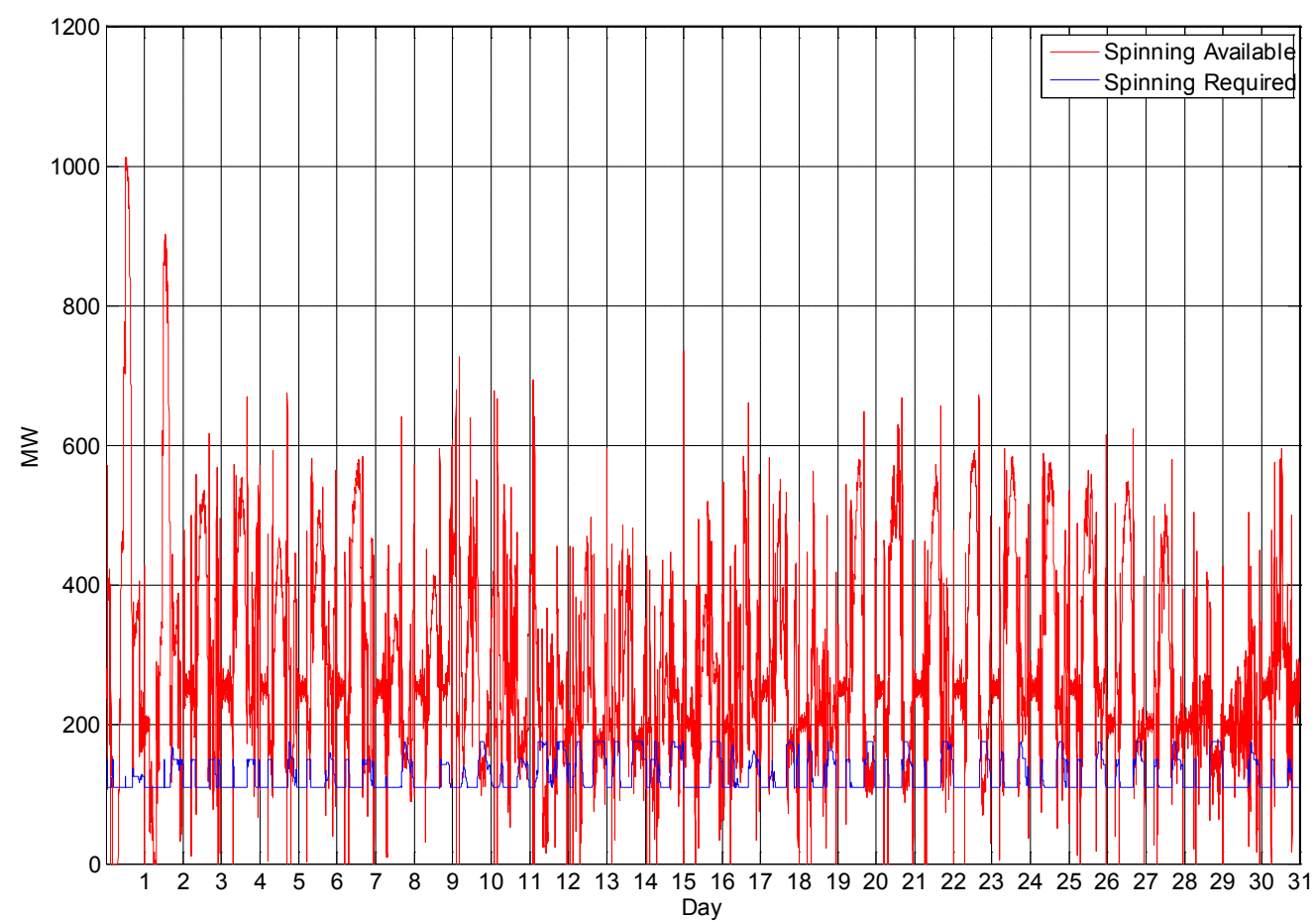

Figure 4.17. Available Spinning Reserve and Spinning Reserve Requirement - January, Case 5A

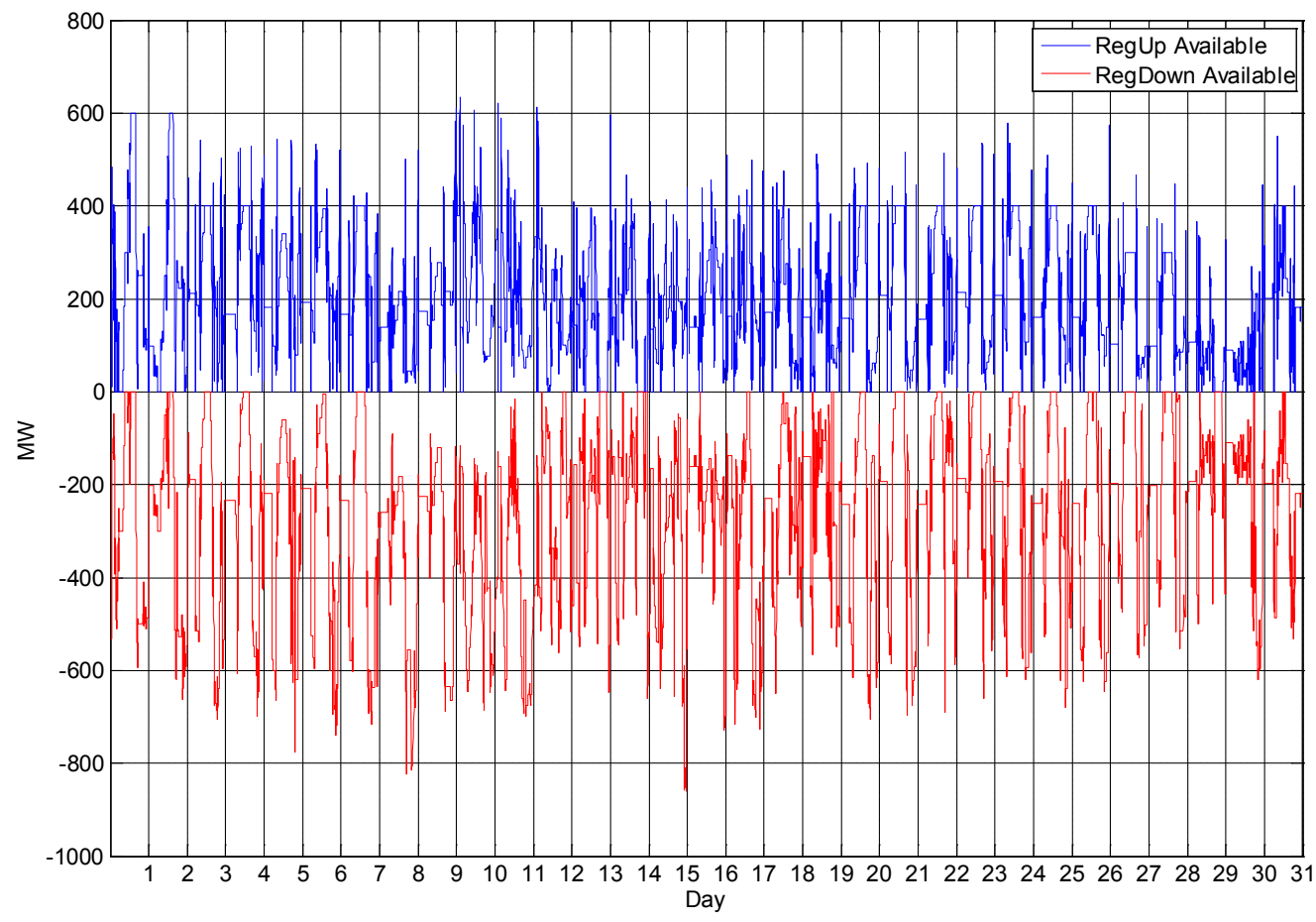

Figure 4.18. Available Regulation Reserves- January, Case 5A 


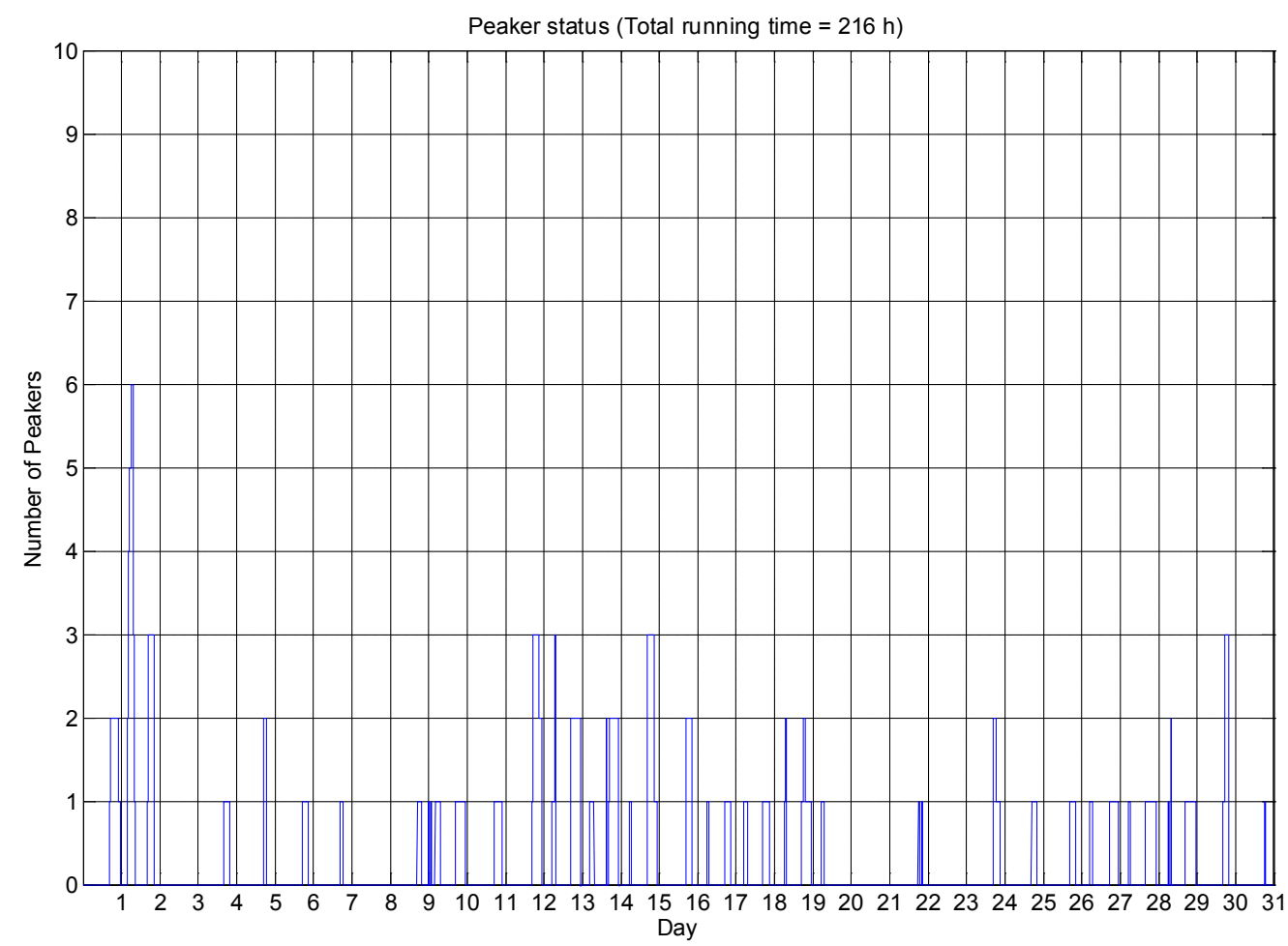

Figure 4.19. Peaking Units Dispatched by the Operator Model in ESIOS - January, Case 5A

It should be noted that besides the characteristics of the generation fleet, such as the combined range of regulation capacity and ramp rate, the unit commitment and scheduling process also has considerable impact on the flexibility of online units. The scheduling software also should be looked at when identifying means to resolve the deficiency of flexible reserves.

\subsubsection{Reserve Deployment - January Case 1A vs. Case 5A}

This section discusses the results on deployed AGC reserves, calculated as the actual total AGC generation subtracted by hourly AGC schedule. Because no contingencies were considered in the simulations, the deployed AGC reserves are only for system balance purpose. The balancing reserve from AGC units can be further separated into regulation and load following components. Consistent with the definition in the 2011 PV integration study [1], load following traces the 10-minute trend of net load, and regulation follows the minute-to-minute variations.

Please note that this separation of fast and slow components of AGC reserves is artificial because no RTD process with a fixed 10-minute interval currently exists in the NV Energy southern system. ${ }^{1}$ Therefore, regulation and load following reserves are differentiated only in this section for the purpose of comparison with the corresponding requirements developed by Lu and co-workers [1]. Regulation reserve and AGC reserve are used interchangeably in other sections to refer to the adjustable range

\footnotetext{
${ }^{1}$ The purpose of this disaggregation is to quantify the need for balancing reserves available at different speeds, or ramp rates. For example, peaking units can be counted to perform load following but not regulation because they can be turned on or off within 10 minutes, but normally do not follow the AGC signal; while the adjustable range of AGC units can be counted toward both regulation and load following reserves because they can be dispatched as needed to chase both fast and slow variations of load.
} 
(upward and downward from the operation point) of AGC units. Figure 4.20 shows the total deployed AGC reserves in Case 1A, and Figure 4.21 shows the load following and regulation reserves derived from the total AGC reserve deployment. Figure 4.22 plots the maximum regulation and load following capacity applied in each hour.

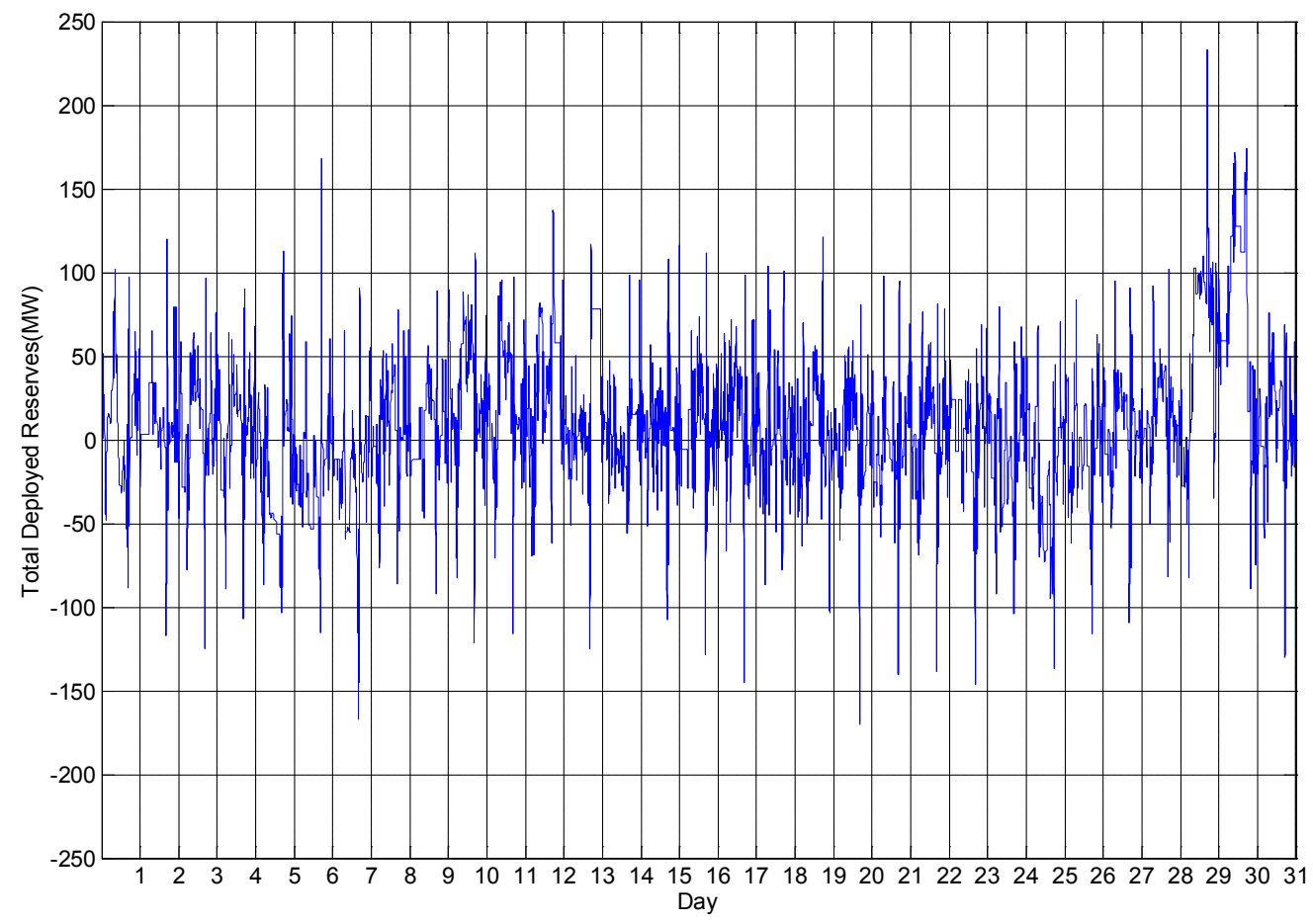

Figure 4.20. Total Deployed AGC Reserves - January, Case 1A

Figure 4.23 to Figure 4.25 show the results of Case 5A corresponding to the above quantities from Case 1A. Please note that in Figure 4.25 the downward load following capacity does not reflect the actual load following needed because the AGC units are on their minimum operation points and cannot be lowered any more (see Section 4.2.2). By comparing Figure 4.22 and Figure 4.25, one can see that the maximum capacity of deployed regulation reserve increases by about $40 \mathrm{MW}$ in both directions from Case 1A to Case 5A; and load following capacity deployment increases much more dramatically in the downward direction than in the upward direction. 

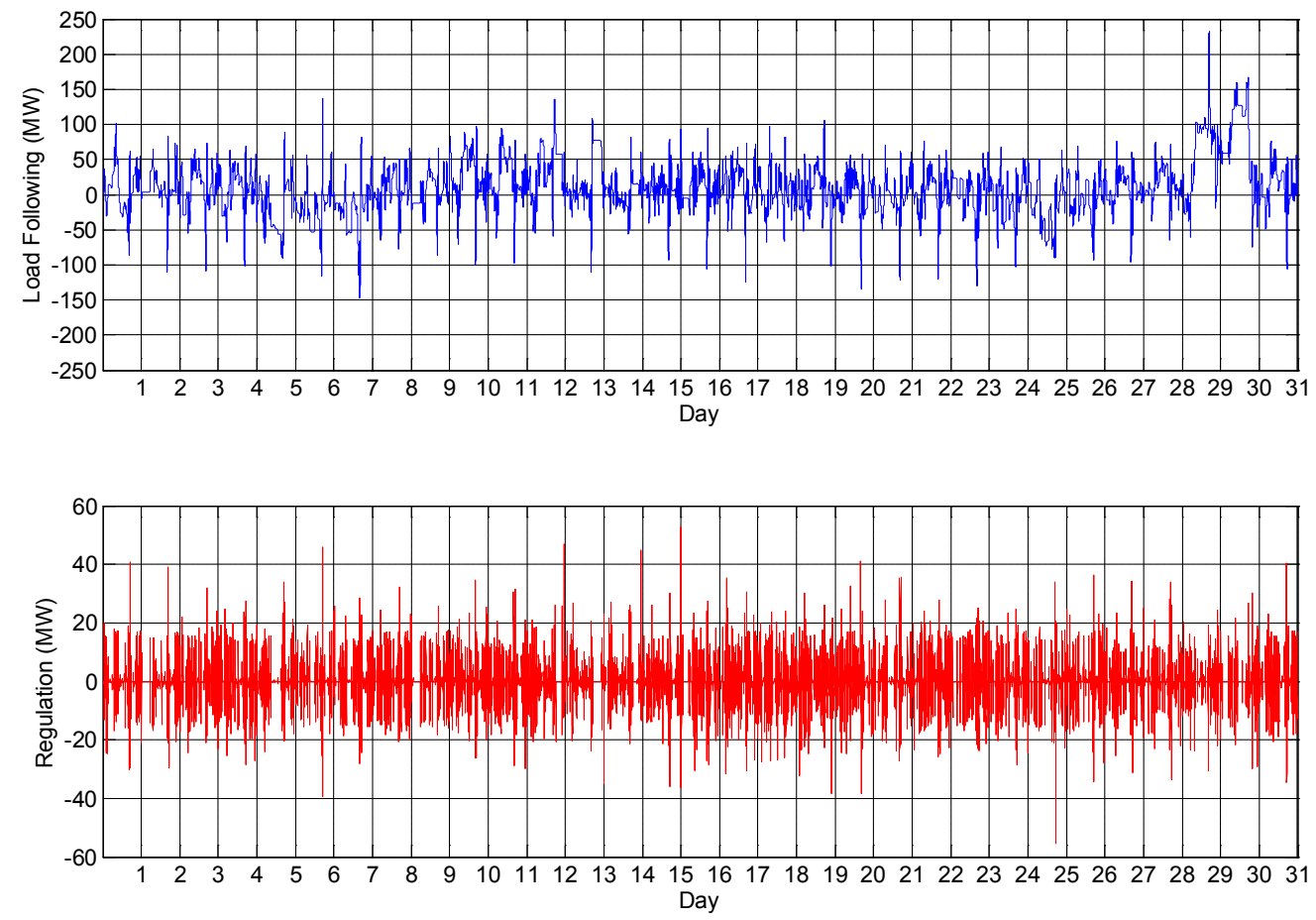

Figure 4.21. Deployed AGC Reserves Separated into Load Following and Regulation Components January, Case 1A
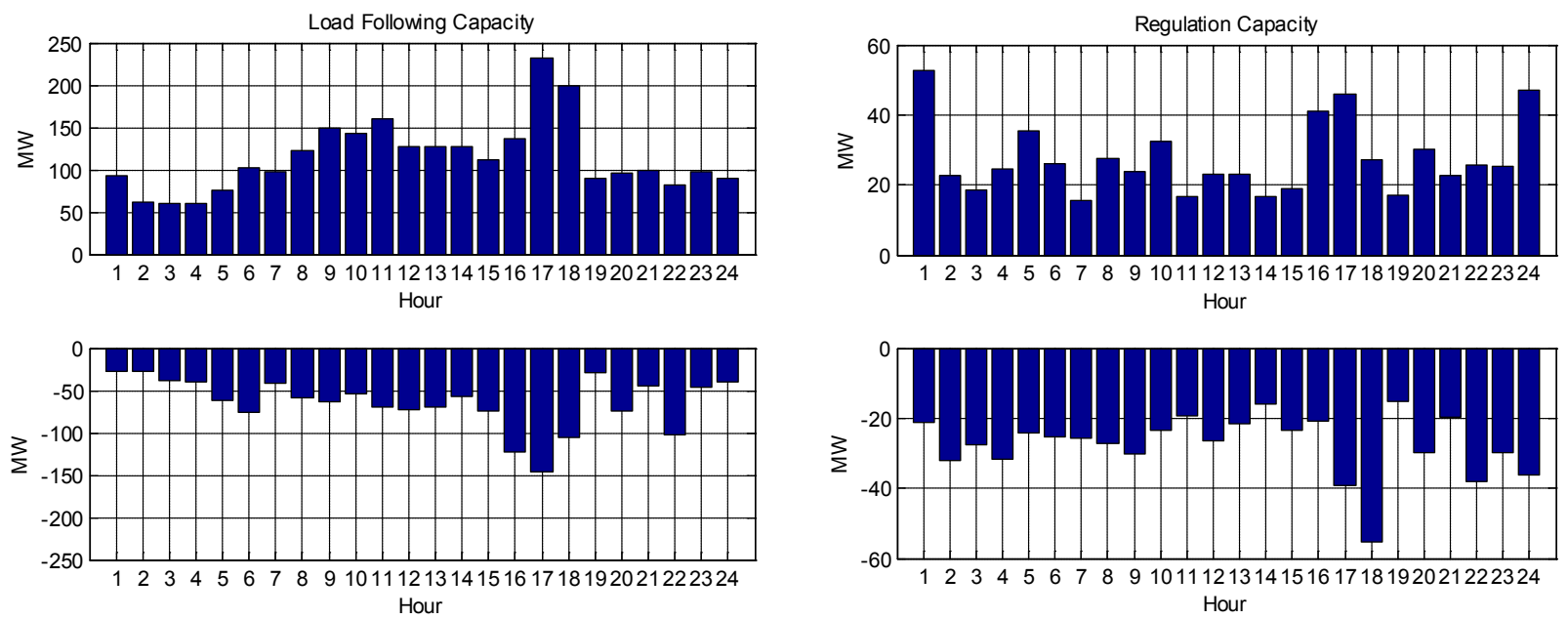

Figure 4.22. Maximum Load Following and Regulation Reserve Deployment by Hour - January, Case $1 \mathrm{~A}$ 


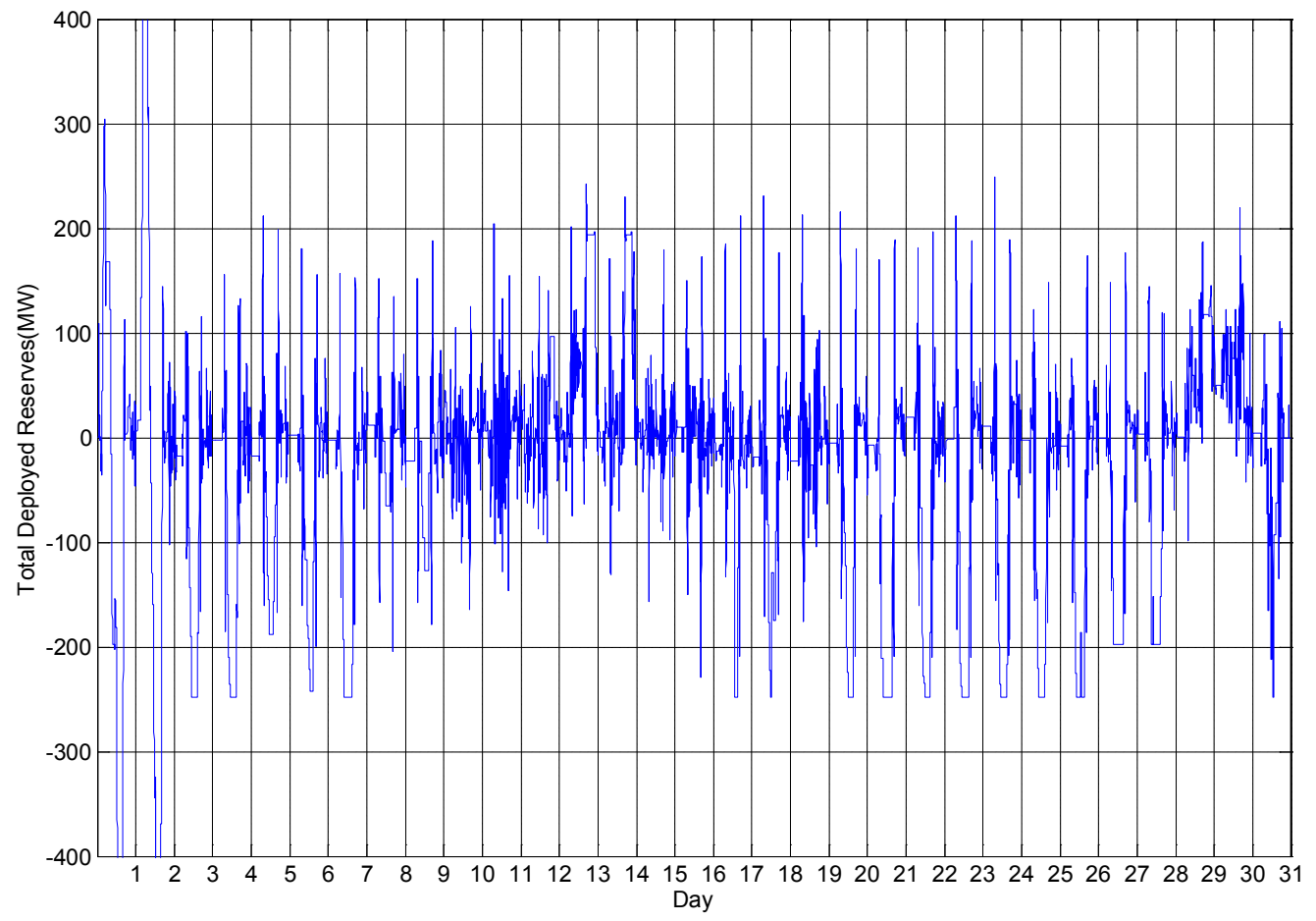

Figure 4.23. Total Deployed AGC Reserves - January, Case 5A
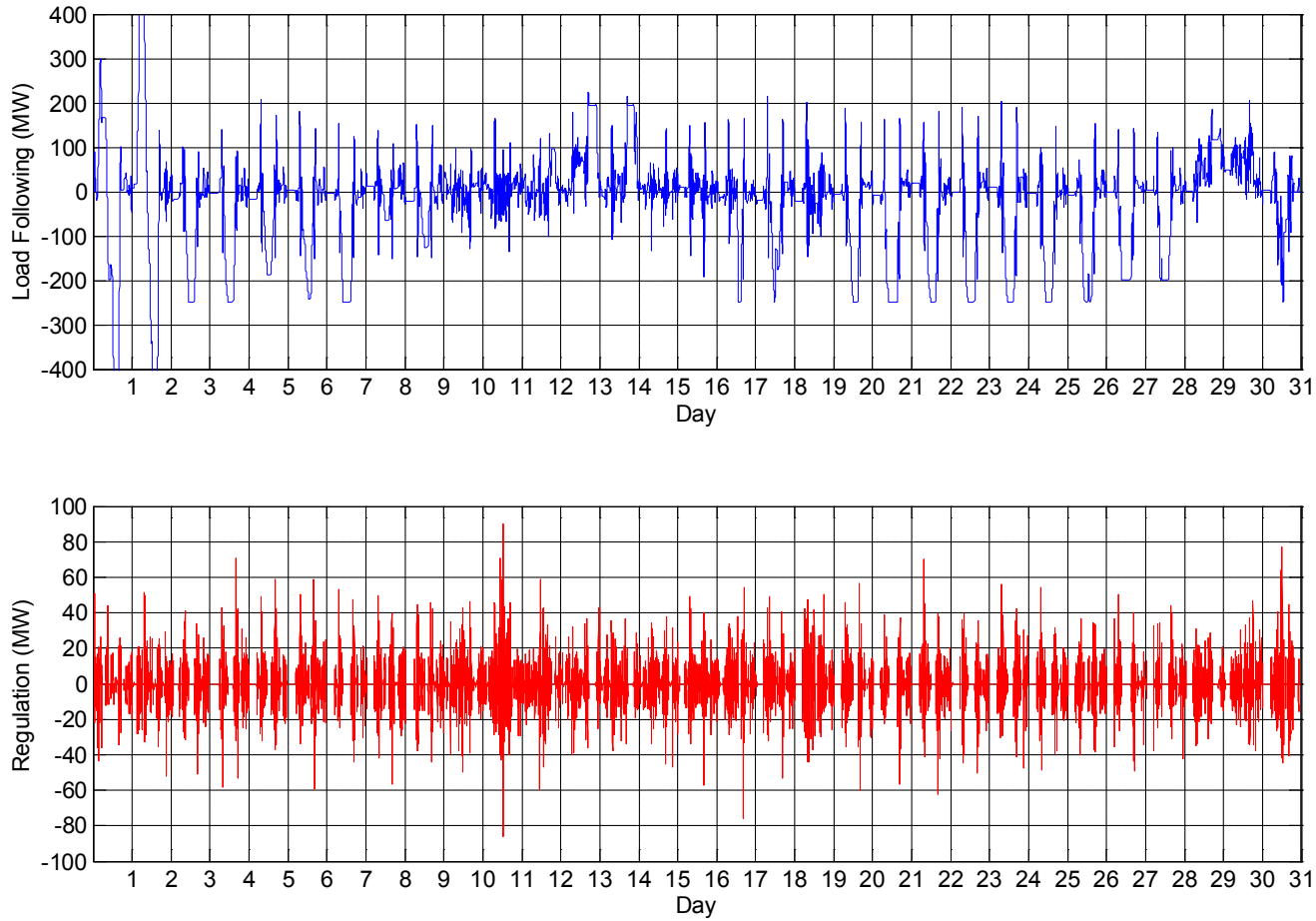

Figure 4.24. Deployed AGC Reserves Separated into Load Following and Regulation Components January, Case 5A 

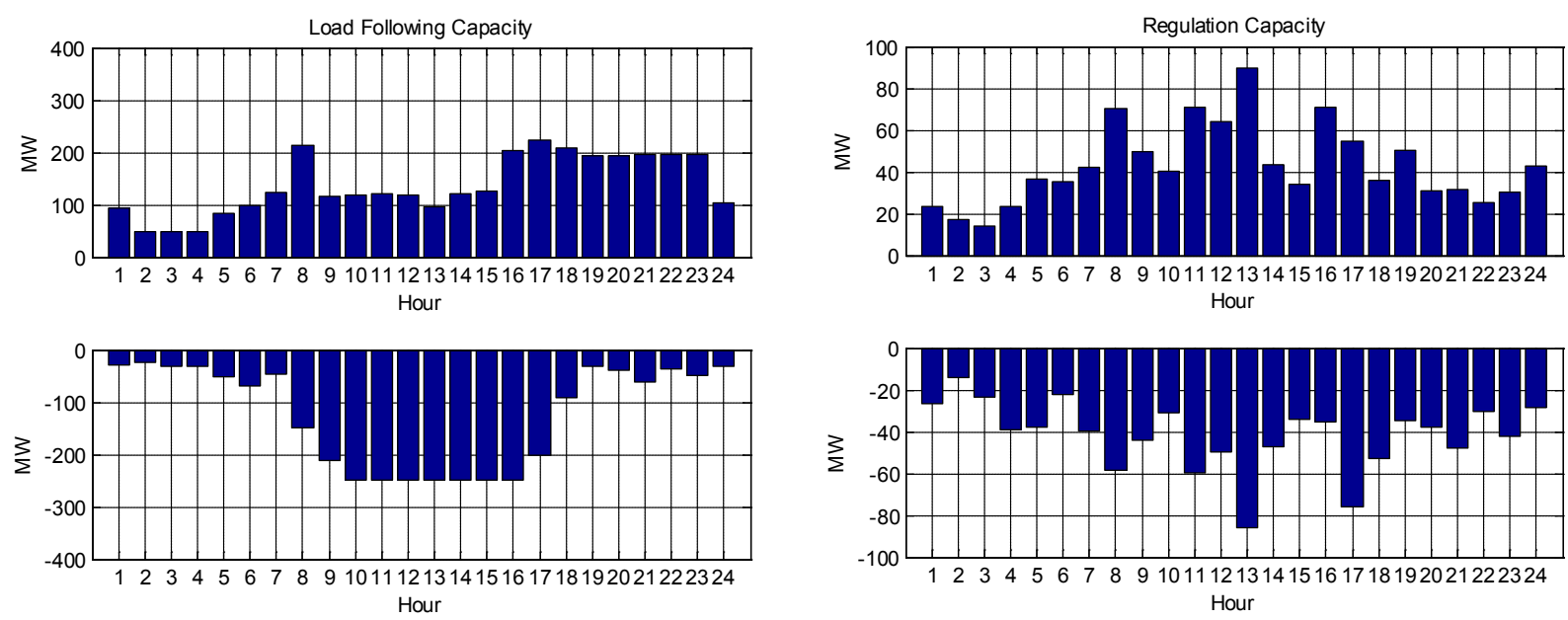

Figure 4.25. Maximum Load Following and Regulation Reserve Deployment by Hour - January, Case 5A

In the PV study in 2011[1], a set of regulation and load following requirements were developed based on the variability of net load. The capacity requirements are redrawn in Figure 4.26 and Figure 4.27. Comparing them with the results in Figure 4.22 and Figure 4.25, one can see that the maximum deployed reserves are actually larger than the requirements from the 2011 study. This can be explained by the fact that the requirements in the 2011 study were derived without consideration of unit commitment and dispatch, generator flexibility, and spinning reserve requirement, as have been included in this study approach.
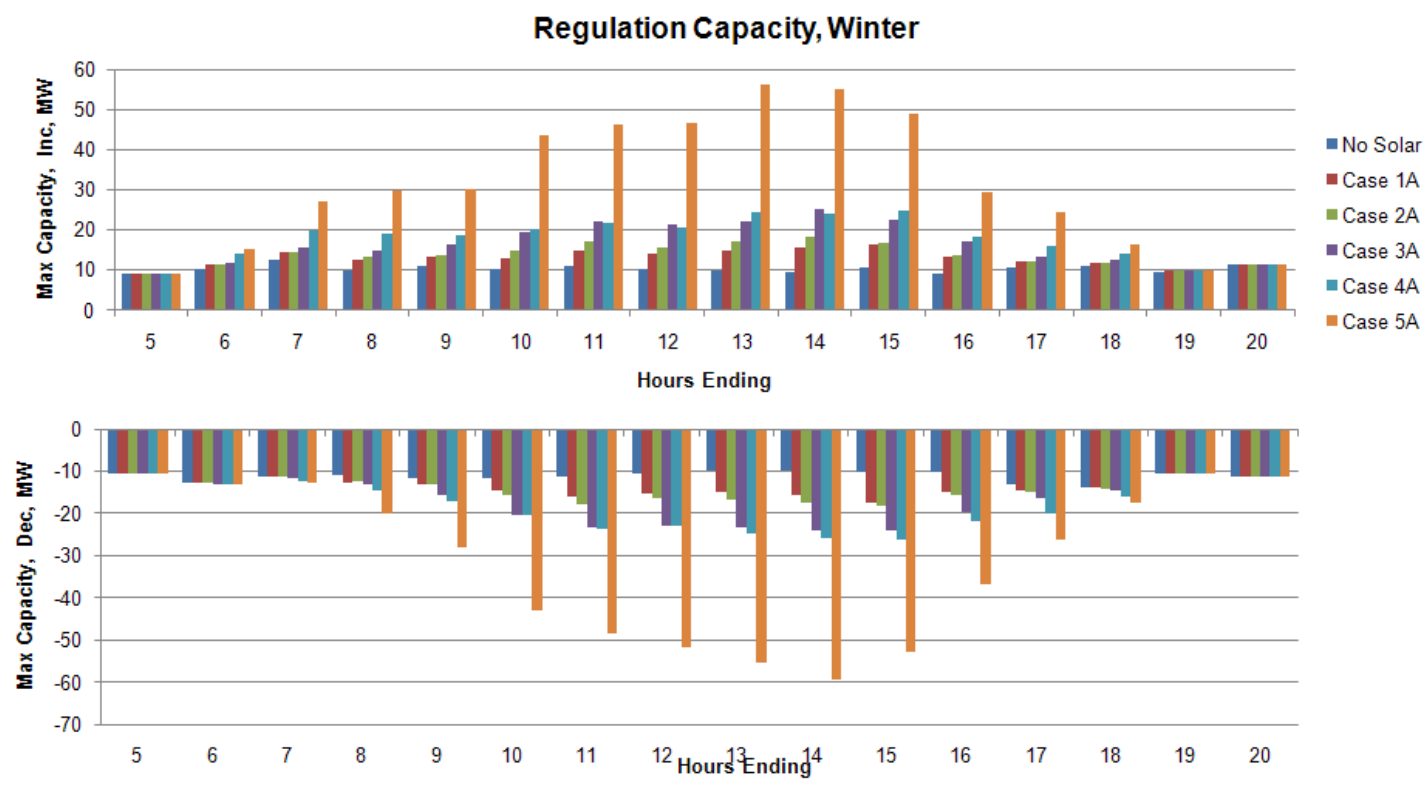

Figure 4.26. Regulation Capacity Requirements - Winter, Cases 1A-5A [1] 

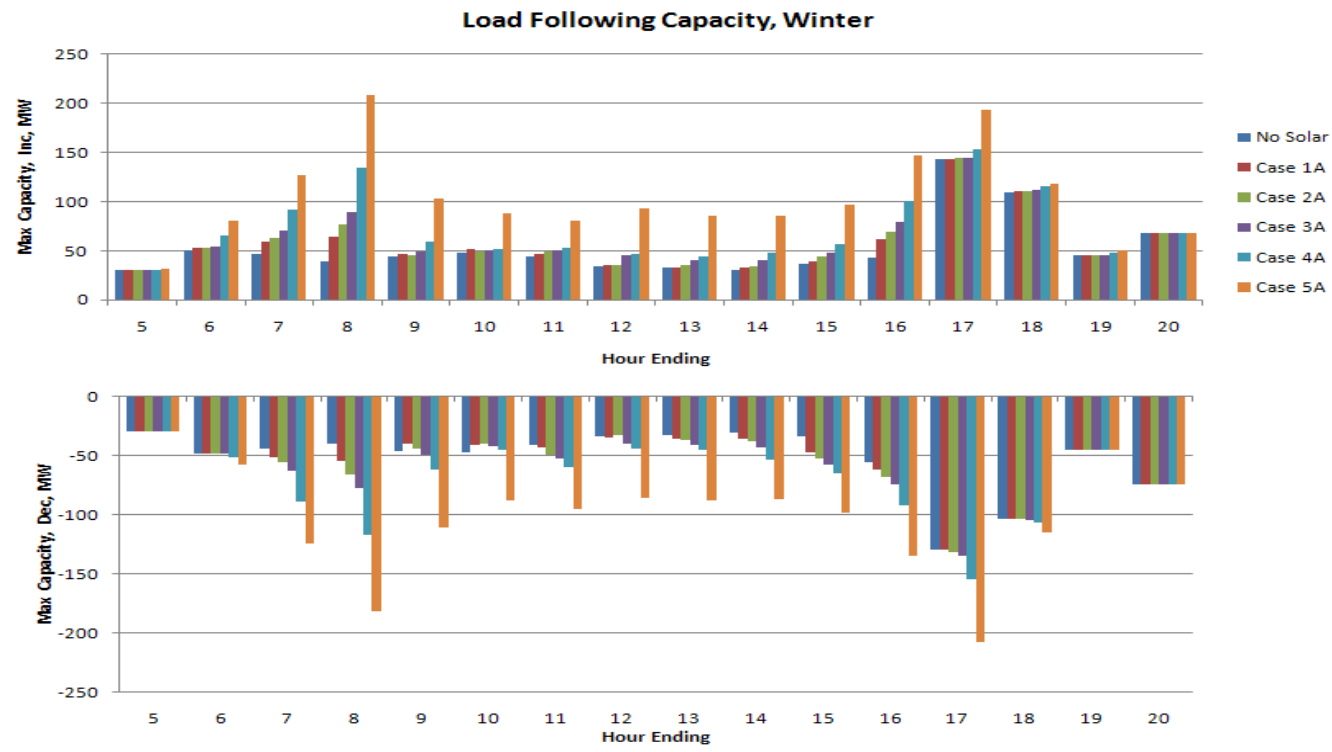

Figure 4.27. Load Following Capacity Requirements - Winter, Cases 1A-5A [1]

\subsubsection{Control Performance - January Case 1A vs. Case 5A}

Figure 4.28 shows the distribution of ACE for January in Case 1A. The CPS2 score is 97 percent. Figure 4.29 and Table 4.2 show the distribution and the statistical characteristics of ACE by hour. The largest positive average of ACE (over generation) is observed in hour 14 and the largest negative (under generation) is observed in hour 6 .

Figure 4.30, Figure 4.31, and Table 4.3 show the corresponding results for Case 5A. The CPS2 score is 91 percent, which still meets the NERC requirement of 90 percent [12], but is much lower than that of Case 1A. The largest positive and negative averages of ACE happen in hour 13 and hour 7, respectively. Both hours see much larger averages and standard deviations on ACE distribution than those in Case 1A, which also indicate deterioration in control performance.

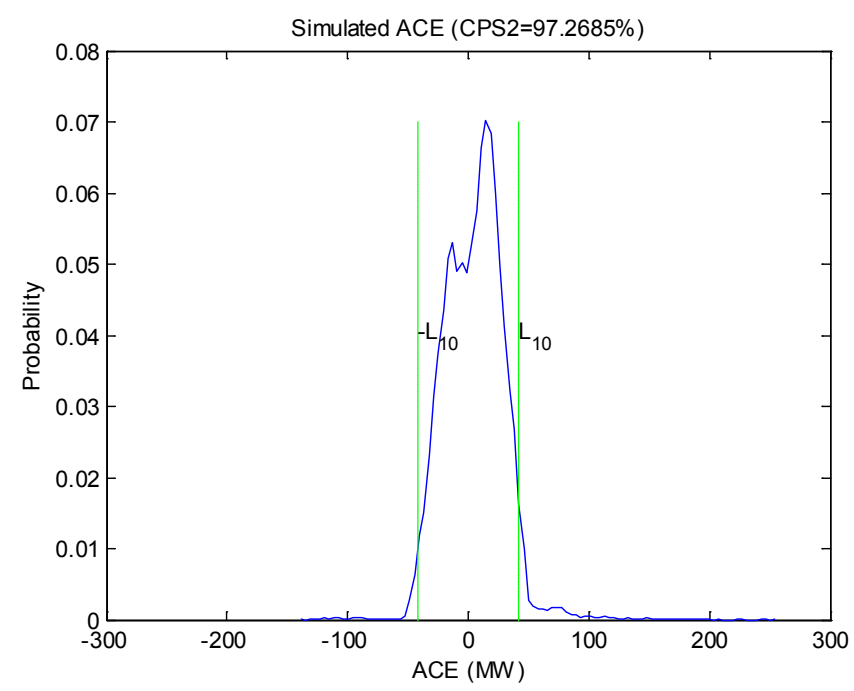

Figure 4.28. ACE Distribution of the Month - January, Case 1A 

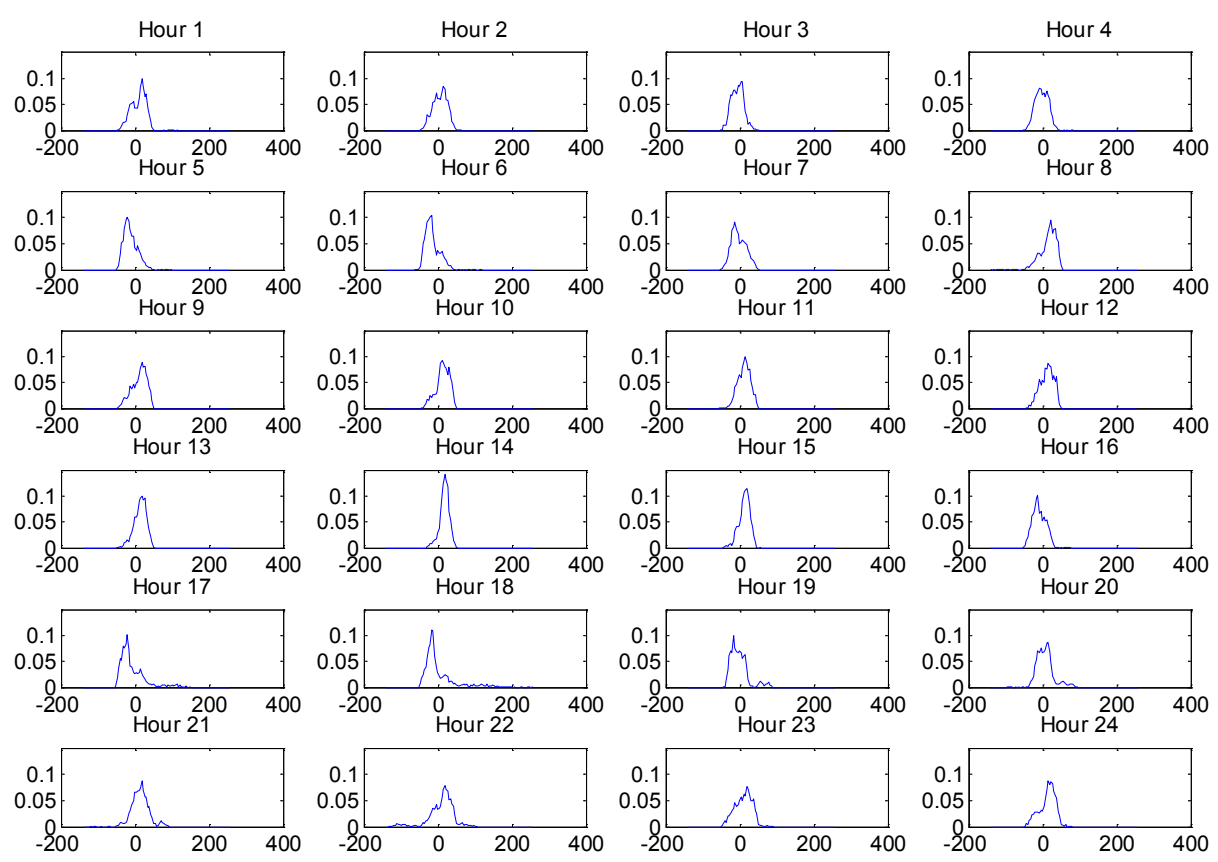

Figure 4.29. ACE Distribution by Hour - January, Case 1A

Table 4.3. ACE Statistical Characteristics by Hour - January, Case 1A

\begin{tabular}{lrrrrrrrrrrrrrrrrrrrrrrrr}
\hline Hour & 1 & 2 & 3 & 4 & 5 & 6 & 7 & 8 & 9 & 10 & 11 & 12 & 13 & 14 & 15 & 16 & 17 & 18 & 19 & 20 & 21 & 22 & 23 & 24 \\
Mean & 9 & 6 & -6 & -2 & -12 & -14 & -3 & 14 & 11 & 13 & 11 & 11 & 14 & 18 & 12 & -9 & -8 & 4 & -3 & 6 & 14 & 10 & 10 & 13 \\
Std & 21 & 19 & 17 & 18 & 20 & 22 & 20 & 23 & 20 & 18 & 17 & 18 & 17 & 13 & 15 & 18 & 35 & 47 & 24 & 24 & 24 & 35 & 23 & 22 \\
\hline
\end{tabular}

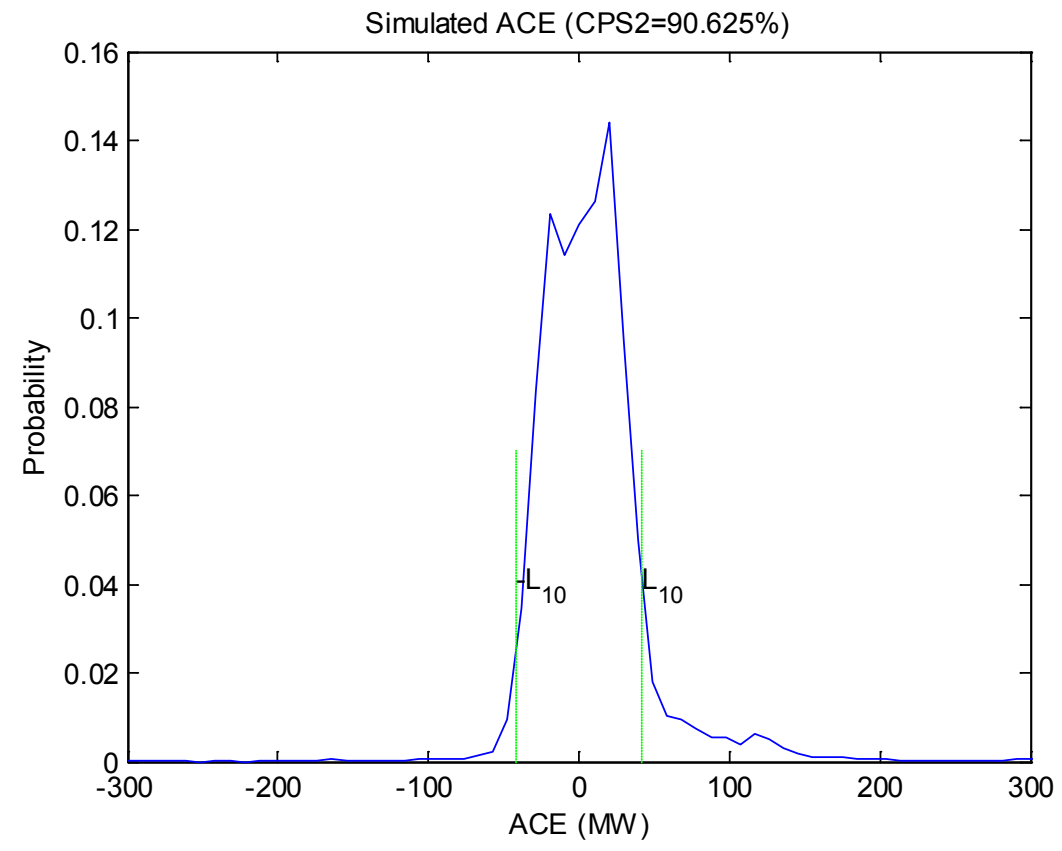

Figure 4.30. ACE Distribution of the Month - January, Case 5A 

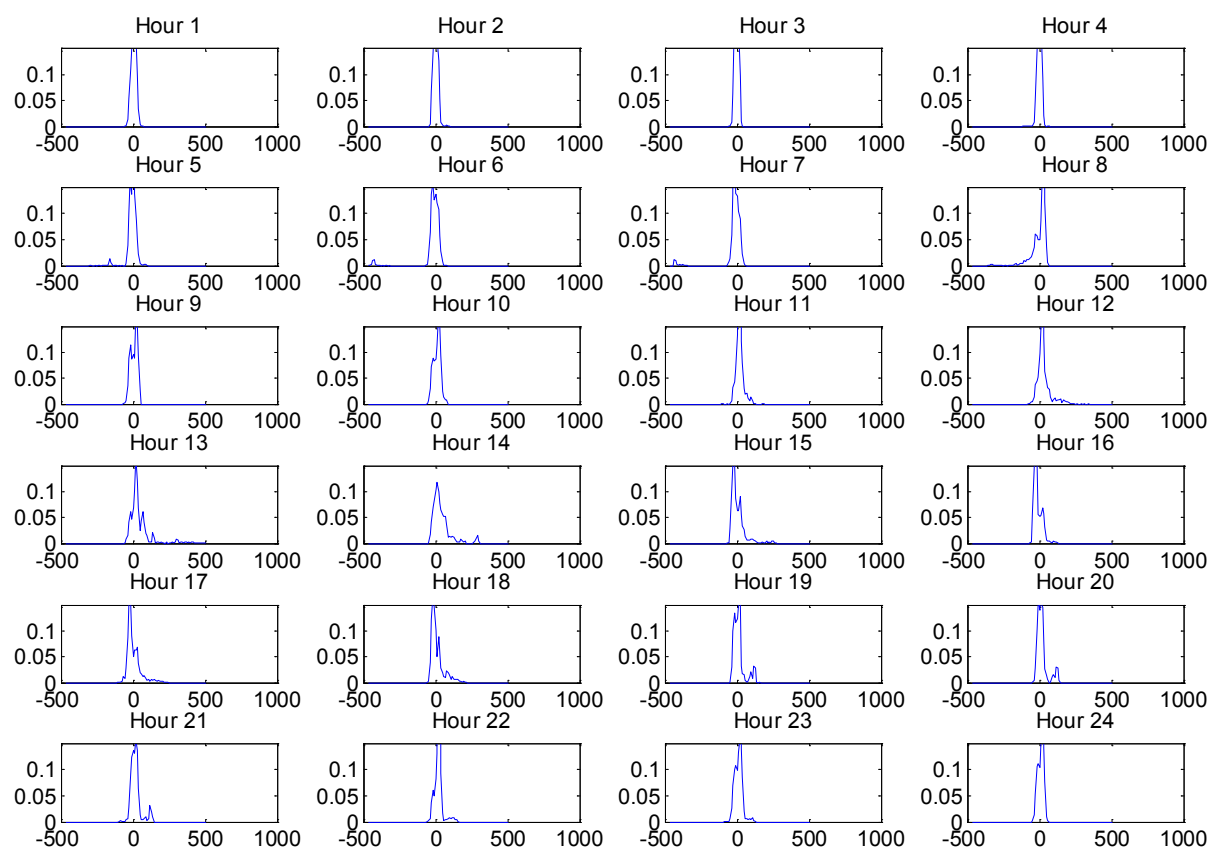

Figure 4.31. ACE Distribution by Hour - January, Case 5A

Table 4.4. ACE Statistical Characteristics by Hour - January, Case 5A

\begin{tabular}{lrrrrrrrrrrrrrrrrrrrrrrrr}
\hline Hour & 1 & 2 & 3 & 4 & 5 & 6 & 7 & 8 & 9 & 10 & 11 & 12 & 13 & 14 & 15 & 16 & 17 & 18 & 19 & 20 & 21 & 22 & 23 & 24 \\
Mean & 4 & 0 & -3 & -2 & -8 & -19 & -21 & -3 & 7 & 11 & 18 & 29 & 47 & 39 & 4 & -12 & 3 & 9 & 12 & 16 & 17 & 21 & 10 & 9 \\
Std & 19 & 17 & 14 & 16 & 37 & 79 & 76 & 56 & 24 & 25 & 27 & 47 & 85 & 64 & 50 & 29 & 48 & 44 & 40 & 37 & 36 & 29 & 27 & 20 \\
\hline
\end{tabular}

\subsection{Results Summary of all PV cases}

Similar results to the examples discussed in Section 4.2 were generated for PV Case 1A to Case 5A. Several indices are established based on the simulation results as measures to evaluate the impact of PV generation on intra-hour operations. These indices include average ACE of the month, CPS2 score, duration of regulation/AGC (up and down) reserve shortage, runtime of peaking units dispatched by the operator model, and adjustments of non-regulation units. The indices measure system control performance, adequacy of fleet capability and involvement of "manual" interventions during the real-time operations. Results from different PV cases then were put together to help identify the patterns caused by the increase of PV generation. All the data used to generate the plots in this section are listed in the tables in Appendix B.

Figure 4.32 shows the average ACE for each month in each study case. While there are no significant differences in Case 1A to Case 4A results, Case 5A shows constant large positive ACE except for the late spring and summer months (May to September, when load is high because of hot weather and tourism). This indicates the potential over generation challenge in fall, winter, and early spring with Case 5A. Similarly, the CPS2 data shown in Figure 4.33 shows worse control performance of Case 5A than the other cases from October through April. The CPS2 scores in October to December also are less than 
90 percent, which will be out of compliance with the current NERC requirement. In the meantime, there is no clear trend of CPS2 scores from Case 1A to Case 4A.

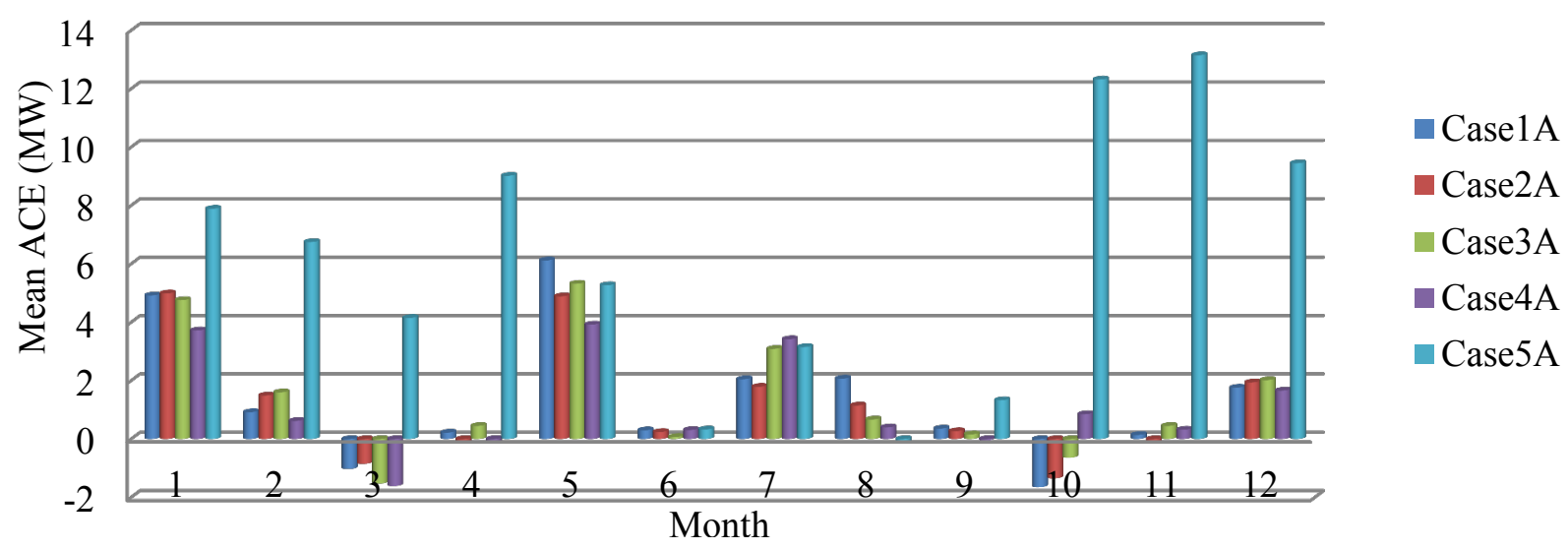

Figure 4.32. Average ACE in Different PV Cases

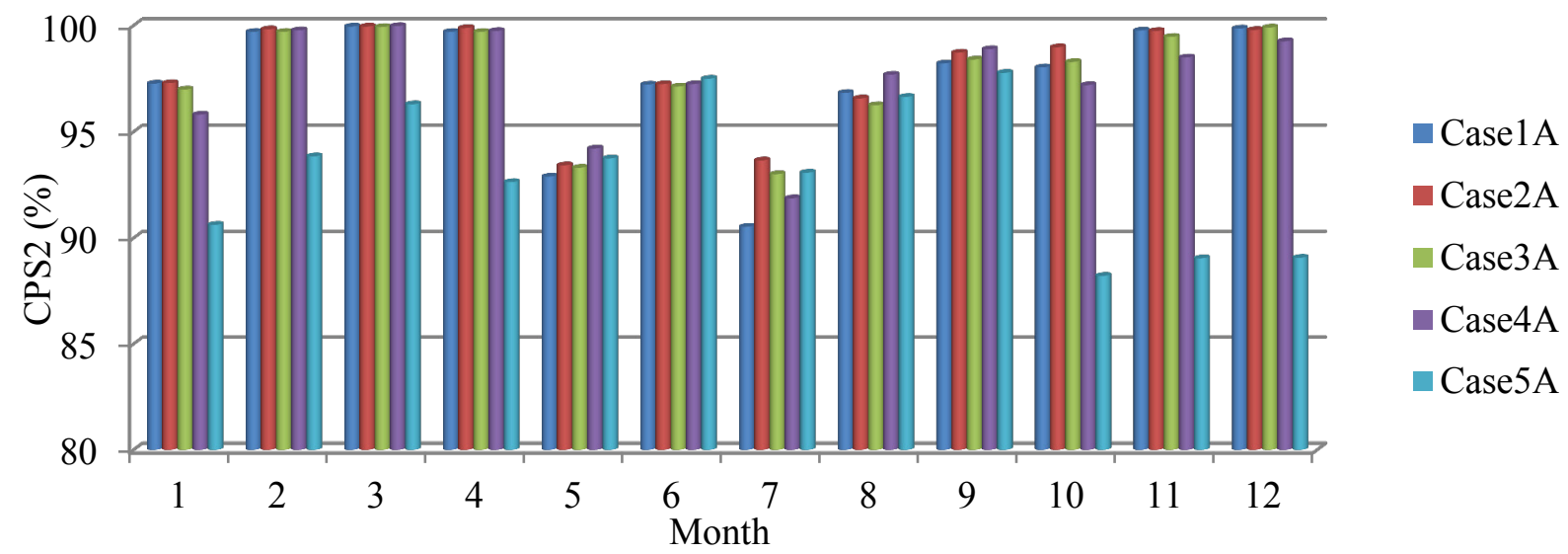

Figure 4.33. CPS2 Performance in Different PV Cases

Figure 4.34 and Figure 4.35 summarize the total time duration when system regulation/AGC up and regulation/AGC down reserves are scarce or nonexixtent. Shortage of RegUp happens most frequently from May to October (except September), but no correlation exists between the duration of shortage and PV penetration level. On the other hand, the duration of the RegDn shortage is significantly higher in Case 5A than other cases from October through April, which is perfectly correlated to large positive ACE and the degradation of CPS2 performance. Similar to the average ACE and control performance statistics, no clear trend can be seen on reserve shortages from Case 1A to Case 4A (other than in one or two specific months). 


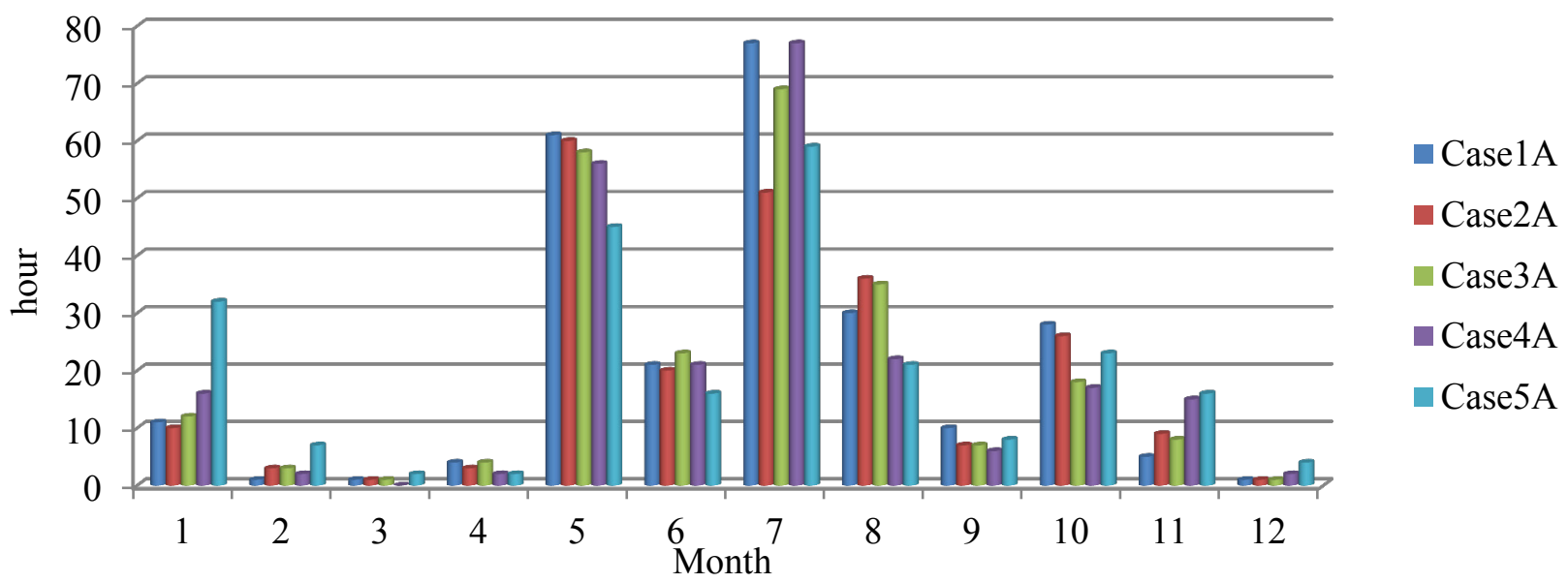

Figure 4.34. Duration of RegUp Reserve Shortage

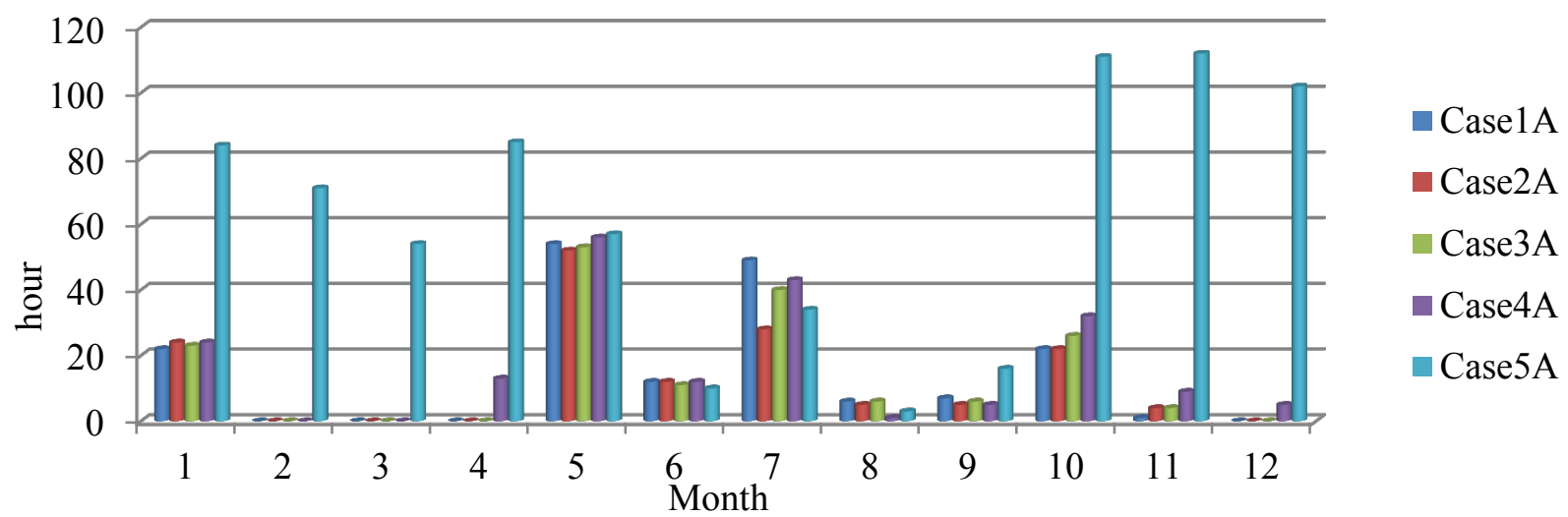

Figure 4.35. Duration of RegDn Reserve Shortage

Runtime of peaking units dispatched by the operator model and the adjustments on non-regulation units are two indices that measure the involvement of "manual" interventions during real-time operations. Figure 4.36 shows the total runtime of peaking units dispatched by the operator model in ESIOS. It is interesting to note that, except for October and December, no other months see the consistent trend of increased peaking unit usage with higher PV penetration. In the months when peaking units are heavily used (i.e., from May to September), the trend is rather the opposite. A reason for this trend follows.

The advantage of peaking units is their flexibility to startup and shutdown quickly (normally within 10 minutes). They are dispatched by scheduling software to meet large load ramps from hour to hour, and by ESIOS to increase upward regulation and spinning reserves. In the high-load season, the ratio between daily peak and minimum load, as shown in Figure 4.37, is much larger than other times; therefore, more peaking units are dispatched to meet the large variation range of load and spinning reserve requirements. For those cases with higher PV penetration, the daily variability of load is more effectively reduced by PV generation, as observed in Figure 4.37, which results in less need for peaking units. In the months with relatively low load (October through April), the difference between daily peak and bottom load is not as significant. Higher PV penetration generally results in larger daily variability range in the net load in this period. Generators are frequently forced to run at minimum load, as manifested by the RegUp shortage in 
Figure 4.35. Peaking units are not needed much during these months because they will only increase upward reserves.

Comparing Figure 4.36 and Figure 4.37, one can observe a very nice correlation between the ways PV influences the runtime of peaking units and the ratio of daily peak to bottom of the net load in each month.

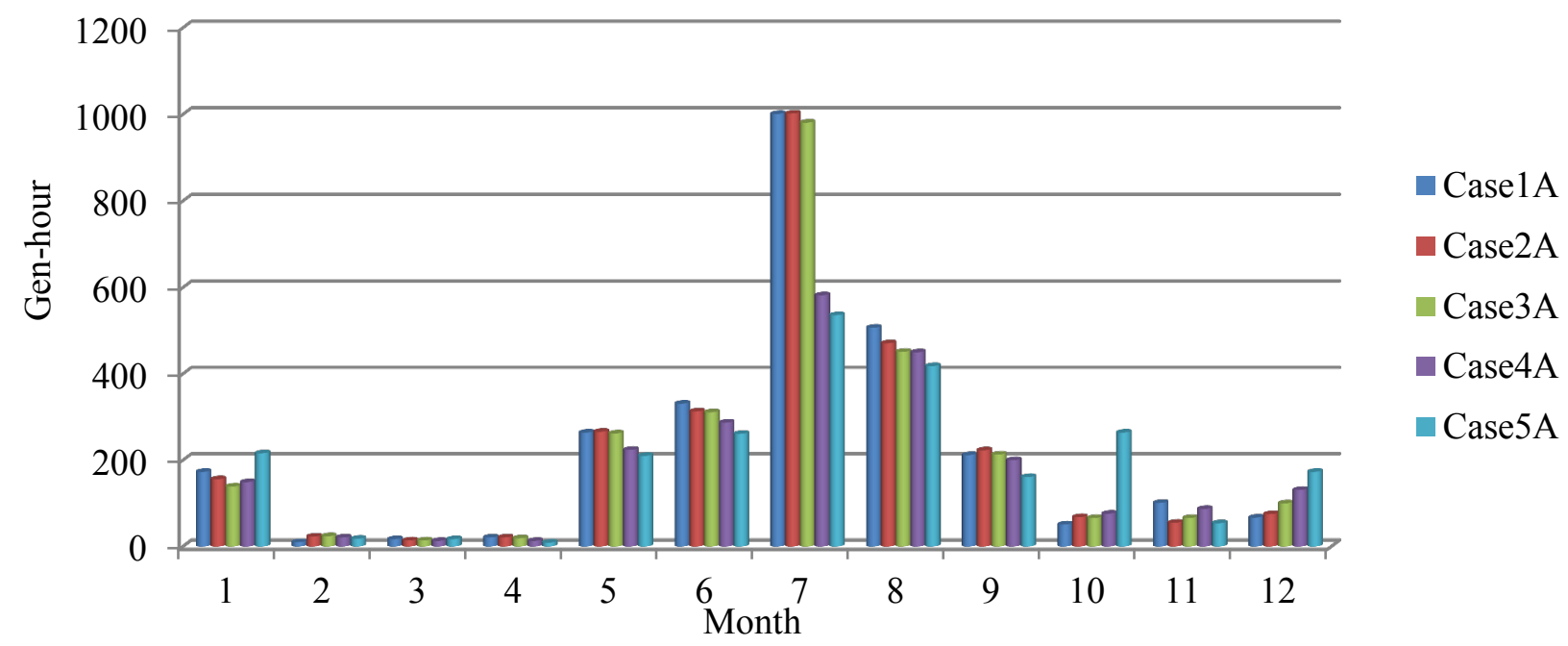

Figure 4.36. Runtime (generator-hour) of Peaking Units dispatched by the Operator Model

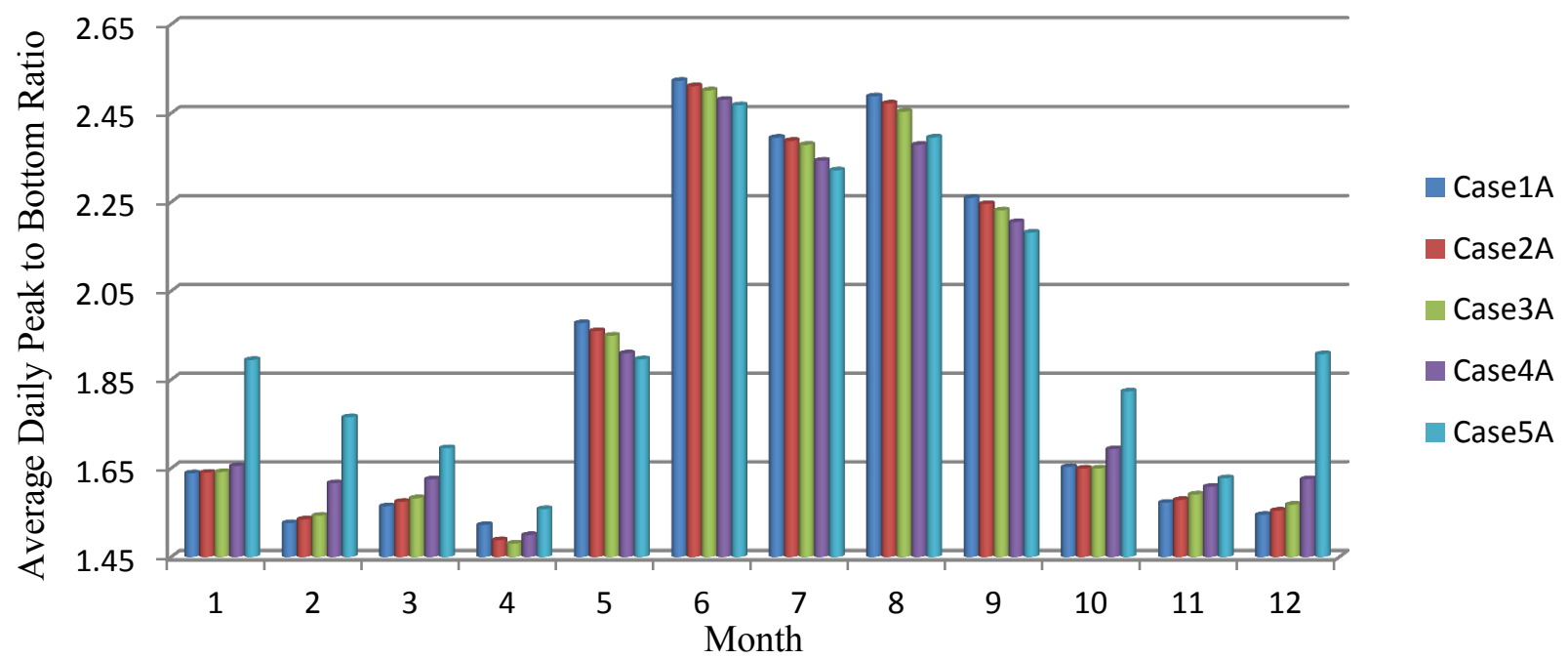

Figure 4.37. Average Ratio of Daily Peak to Bottom of Net Load

Figure 4.38 provides statistics of operator "manual" dispatch action on non-regulation generators. The operator model would dispatch AGC units that are not in regulation mode to help increase regulation reserves, as described in Section 2.4.1. Any hour, during which the operator model takes such an action on a generator is counted as a generator-hour. One can see that from October through March, the dispatch 
actions increase slightly with PV penetration, while from June to September the trend is the opposite. This observation could also be attributed to the seasonal load and PV patterns of the system.

One caution to readers is that a number of parameters can be adjusted in the operator model to change the results in peaking units runtime and manual dispatch of non-regulation units, such as the time horizon used to estimate available reserves and the trend of load demand (see Section 2.4 for details of the operator model). Because of the constraint of time, the robustness of the above observations against these parameters is yet to be tested.

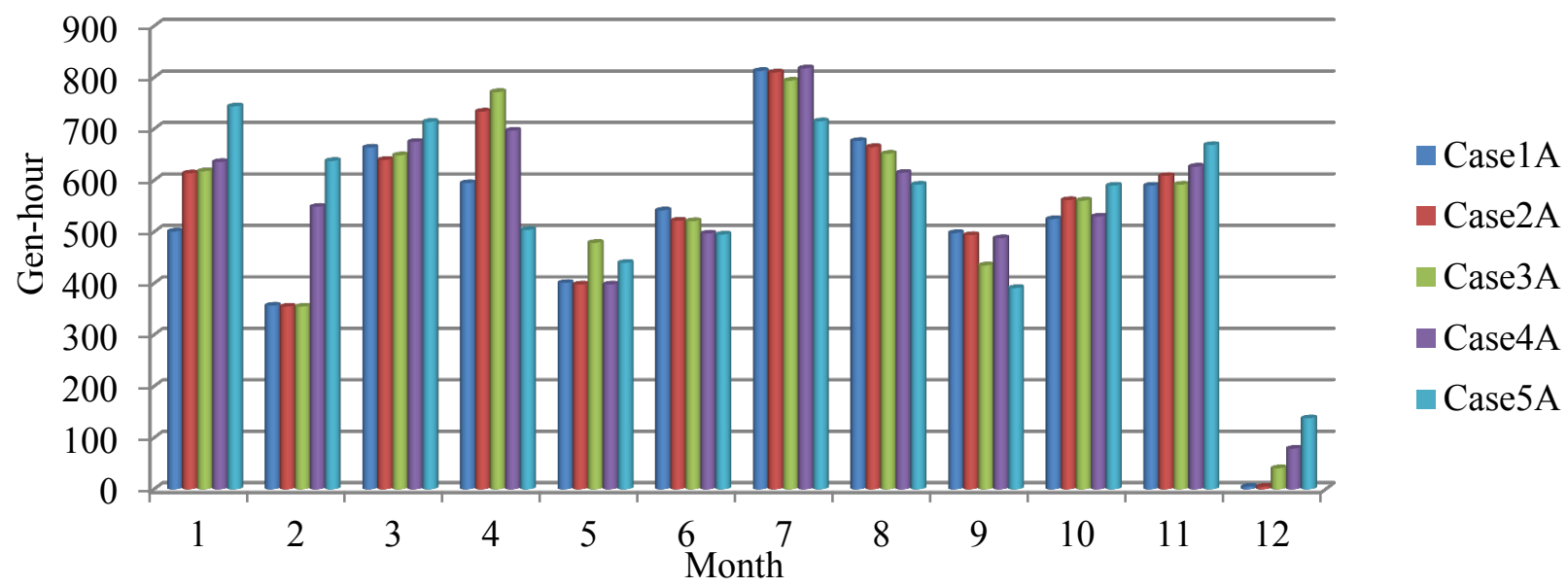

Figure 4.38. Operator Manual Dispatch (generator-hour) on Non-Regulation Units

We can summarize the impact of PV generation on the NV Energy southern system operations as follows:

1. The impact of PV generation on system AGC reserve shortages, ACE distribution, and CPS2 performance shows two different patterns depending on time of the year:

- From October through the winter season and to April when the system load is relatively low, Case 5A sees significantly higher values on average ACE, duration of downward AGC reserve shortage, and much deteriorated CPS2 score compared to other cases. The change of these quantities from Case 1A to Case 4A shows an upward trend in some time but the increases are relatively negligible.

- From May to September, when the system load is relatively high because of hot weather and increased tourism activities in the area, average ACE, duration of AGC reserve shortage. and CPS2 score are not impaired by the increase of PV generation. In many cases, the system exhibited better CPS performance with increased PV generation.

2. Operator manual dispatch of peaking units and non-regulation units show similar seasonal effects with the increase of PV generation as in item 1 above. However, the differences between Case 5A and other cases on these two quantities are much less significant than the quantities in item 1.

Based on ACE and CPS2, it appears that the NV Energy southern system fleet would be capable of handling PV generation in quantities as large as found in Case 4A, with appropriate operator actions (under the perfect forecasts condition), although there are periods that AGC reserves are exhausted. 
Maintaining CPS2 compliance is very challenging for Case 5A during the low-load season (October through April), mainly because of the lack of downward reserve. It needs to be pointed out that besides the characteristics of the generation fleet, the unit commitment and scheduling process also has

considerable impact on the flexibility range of online generation units. The scheduling software also should be looked at when identifying means to resolve the deficiency of flexible reserves.

Because the current study used actual load and PV generation data to perform the simulations, the impacts described above are solely attributed to the inherent variability of load and PV. With imperfect forecasts, the challenges imposed by PV generation are anticipated to be a lot greater. The impact of forecast uncertainty should be studied as data become available. 


\subsection{Conclusions and Future Work}

This report describes a software tool developed by PNNL for simulating power system intra-hour operations. The tool, ESIOS, was applied on the NV Energy southern system and simulated both the AGC process and operator manual actions. Comparison with historical AGC data shows that ESIOS is able to approximate actual system behaviors satisfactorily after appropriate tuning of simulation parameters.

Five different PV penetration cases (Case 1A to Case 5A), which contain large-scale PV installations ranging from $149 \mathrm{MW}$ to $892 \mathrm{MW}$ and distributed PV installation of $50 \mathrm{MW}$, were then studied using the simulator. The impact of PV generation in these study cases on system intra-hour operations and control performance can be summarized as follows:

1. The impact of PV generation on system AGC reserves shortages, ACE distribution, and CPS2 performance shows two different patterns depending on time of the year:

- From October through the winter season and to April when the system load is relatively low, Case 5A (892 MW large PV and 50 MW DG) sees significantly higher values for average ACE, duration of downward AGC reserve shortage, and a much deteriorated CPS2 score compared to other cases. The change of these quantities from Case 1A (149 MW large PV and $50 \mathrm{MW}$ DG) to Case 4A (492 MW large PV and 50 MW DG) shows an upward trend in some time but the increases are relatively negligible.

- From May to September, when the system load is relatively high because of hot weather and increased tourism activities in the area, average ACE, duration of AGC reserves shortages, and CPS2 score are not impaired by the increase of PV generation. In many cases, with increased PV generation, the CPS performance exhibited some improvement.

2. Operator manual dispatch of peaking units and non-regulation units show similar seasonal effects with the increase of PV generation as in item 1 above. In the low-load season (October through April), higher PV penetration results in larger daily variability ranges of net load and consequently more operator intervention (dispatch of peaking units and non-regulating units). In the high-load season (May to September), the trend is reversed because PV generation counteracts the increased daytime peak and results in lower variability ranges of net load. On the other hand, the differences between Case 5A and other cases with respect to operator intervention are much less significant than the quantities in item 1.

Based on ACE and CPS2, it appears that the NV Energy southern system fleet would be capable of handling PV generation in quantities as large as is found in Case 4A, with appropriate operator actions (under the perfect forecasts condition), although there are periods that AGC reserves are exhausted. Maintaining CPS2 compliance is very challenging for Case 5A during the low-load season (October through April), mainly because of the lack of downward reserve. Besides the characteristics of the generation fleet, the unit commitment and scheduling process also has considerable impact on the flexibility range of online generation units. The scheduling software should also be looked at when identifying means to resolve the deficiency of flexible reserves.

Because the current study used actual load and PV generation data to perform the simulations, the impacts described previously are solely attributed to the inherent variability of load and PV. With 
imperfect forecasts, the challenges imposed by PV generation are anticipated to be much greater. Those months when CPS 2 barely exceeds the 90 percent threshold may not be able to comply with the requirement if forecast errors are considered. The impact of forecast uncertainty should be studied as data become available.

Many parameters in the AGC and operator models can affect the simulation results. For example, the look-ahead time horizon for estimating available reserves can affect the number of peaking units started. Because of time limits, not all parameters were thoroughly investigated to test their influence on the results. The robustness of the conclusion above is still subject to more testing and simulations.

In the real world, system operators may have more means and flexibility to adjust generator output or start up/shut down generators to improve control performance, than the functions simulated in the operator model in ESIOS. Nevertheless, an easy solution is not always guaranteed; for example, when the shortage of downward regulation reserve happens in late afternoon (as observed in January Case 5A), generators cannot simply be shut down, because they have to prepare for the coming evening peak. Strategies need to be developed, or technologies such as storage and DR adopted, to help operators prepare for these situations when PV penetration reaches a certain level. ESIOS can be used as a highfidelity test bed for these strategies or technologies during the development process. 


\subsection{References}

[1] Lu S, PV Etingov, R Diao, J Ma, NA Samaan, YV Makarov, X Guo, RP Hafen, C Jin, H Kirkham, E Shlatz, L Frantzis, T McClive, G Karlson, D Acharya, A Ellis, J Stein, C Hansen, V Chadliev, M Smart, R Saigo, R Sorensen, B Allen, and B Idelchik. 2011. Large-Scale PV Integration Study. PNNL-20677, Pacific Northwest National Laboratory, Richland, Washington. Available at http://www.pnnl.gov/main/publications/external/technical_reports/PNNL-20677.pdf.

[2] Ma J, S Lu, RP Hafen, PV Etingov, YV Makarov, and V Chadliev. 2012. "The Impact of Solar Photovoltaic Generation on Balancing Requirements in the Southern Nevada System." IEEE Transmission and Distribution Conference 2012, Orlando, Florida, May 6-10, 2012.

[3] Etingov P, S Lu, X Guo, J Ma, YV Makarov, and V Chadliev. 2012. "Identifying Challenging Operating Hours for Solar Integration in the NV Energy System." IEEE Transmission and Distribution Conference 2012, Orlando, Florida, May 6-10, 2012.

[4] Diao R, S Lu, and J Ma. 2012. "On Evaluating Cycling and Movement of Conventional Generators for Balancing Services with Large Solar Penetration.” IEEE Transmission and Distribution Conference 2012, Orlando, Florida, May 6-10, 2012.

[5] Diao R, S Lu, P Etingov, YV Makarov, J Ma, and X Guo. 2011. NV Energy Solar Integration Study: Cycling and Movements of Conventional Generators for Balancing Services. PNNL-20594, Pacific Northwest National Laboratory, Richland, Washington. Available at http://www.pnnl.gov/main/publications/external/technical_reports/PNNL-20594.pdf

[6] Henderson PD, H Klaiman, J Ginnetti, T Snodgrass, N Cohn, S Bloor, and L VanSlyck. 1990. "Cost Aspects of AGC, Inadvertent Energy and Time Error." IEEE Transactions on Power Systems 5(1):111-118.

[7] Jaleeli N, LS VanSlyck, DN Ewart, and LH Fink. 1992. "Understanding Automatic Generation Control." IEEE Transactions on Power Systems 7(3):1106-1122.

[8] Prowse DCH. 1993. "Improvements to a Standard Automatic Generation Control Filter Algorithm." IEEE Transactions on Power Systems 8(3):1204-1210.

[9] Yao M, RR Shoults, and R Kelm. 2000. "AGC Logic Based on NERC's New Control Performance Standard and Disturbance Control Standard." IEEE Transactions on Power Systems 15(2):852-857.

[10] Kumar IP and DP Kothari. 2005. "Recent Philosophies of Automatic Generation Control Strategies in Power Systems.” IEEE Transactions on Power Systems 20(1):346-357.

[11] Open Systems International (OSI) Operator Training Guide and other AGC system materials provided by OSI and NV Energy. 
[12] NERC - North American Electric Reliability Corporation. 2007. Performance Standards Reference Document. Available at http://www.nerc.com/docs/oc/rs/Item_4ePSRD_revised_112607.pdf. 
Appendix A

AGC Units Characteristics 



\section{Appendix A}

\section{AGC Units Characteristics}

Table A.1. Combine Cycle AGC Generator Characteristic Table

\begin{tabular}{lcccrrrrr}
\hline & & Config & & & & \multicolumn{2}{c}{ Ramp } \\
Unit Name & Unit ID & Name & Config Name Alias & Pmin & Pmax & Rgmin & Rgmax & Rate \\
\hline CC Unit A & 1 & 1 & $1 \times 1$ & 150 & 250 & 150 & 250 & 8 \\
CC Unit A & 1 & 2 & $2 \times 1$ & 250 & 500 & 250 & 500 & 16 \\
CC Unit A & 1 & 3 & $2 \times 1,1 \mathrm{DB}$ & 500 & 525 & 0 & 0 & 4 \\
CC Unit A & 1 & 4 & $2 \times 1,2 \mathrm{DB}$ & 525 & 550 & 0 & 0 & 4 \\
CC Unit B & 2 & 1 & $1 \times 1$ & 150 & 250 & 150 & 250 & 8 \\
CC Unit B & 2 & 2 & $2 \times 1$ & 250 & 500 & 250 & 500 & 16 \\
CC Unit B & 2 & 3 & $2 \times 1,1 \mathrm{DB}$ & 500 & 525 & 0 & 0 & 5 \\
CC Unit B & 2 & 4 & $2 \times 1,2 \mathrm{DB}$ & 525 & 550 & 0 & 0 & 5 \\
CC Unit C & 3 & 1 & $1 \times 1$ & 150 & 250 & 150 & 250 & 7.5 \\
CC Unit C & 3 & 2 & $2 \times 1$ & 250 & 500 & 250 & 500 & 15 \\
CC Unit C & 3 & 3 & $2 \times 1,1 \mathrm{DB}$ & 500 & 525 & 0 & 0 & 4 \\
CC Unit C & 3 & 4 & $2 \times 1,2 \mathrm{DB}$ & 525 & 550 & 0 & 0 & 4 \\
CC Unit D & 4 & 1 & $1 \times 1$ & 150 & 250 & 150 & 250 & 7.5 \\
CC Unit D & 4 & 2 & $2 \times 1$ & 250 & 500 & 250 & 500 & 15 \\
CC Unit D & 4 & 3 & $2 \times 1,1 \mathrm{DB}$ & 500 & 525 & 0 & 0 & 4 \\
CC Unit D & 4 & 4 & $2 \times 1,2 \mathrm{DB}$ & 525 & 550 & 0 & 0 & 4 \\
CC Unit E & 5 & 1 & $1 \times 1$ & 150 & 250 & 0 & 0 & 6 \\
CC Unit E & 5 & 2 & $2 \times 1$ & 250 & 500 & 250 & 500 & 12 \\
CC Unit E & 5 & 3 & $2 \times 1,1 \mathrm{DB}$ & 500 & 525 & 0 & 0 & 6 \\
CC Unit E & 5 & 4 & $2 \times 1,2 \mathrm{DB}$ & 525 & 550 & 0 & 0 & 6 \\
\hline
\end{tabular}



Appendix B

Summary of Simulation Results of Case 1A to Case 5A 



\section{Appendix B}

\section{Summary of Simulation Results of Case 1A to Case 5A}

Table B.1. Average ACE by Month

\begin{tabular}{ccccccccccccc}
\hline Month & Jan & Feb & Mar & Apr & May & Jun & Jul & Aug & Sep & Oct & Nov & Dec \\
\hline Case 1A & 4.92 & 0.92 & -1.03 & 0.22 & 6.12 & 0.3 & 2.05 & 2.07 & 0.36 & -1.65 & 0.14 & 1.76 \\
Case 2A & 4.99 & 1.49 & -0.85 & -0.12 & 4.89 & 0.24 & 1.79 & 1.15 & 0.27 & -1.35 & -0.1 & 1.94 \\
Case 3A & 4.76 & 1.6 & -1.53 & 0.45 & 5.32 & 0.07 & 3.09 & 0.67 & 0.16 & -0.64 & 0.45 & 2.02 \\
Case 4A & 3.72 & 0.62 & -1.61 & -0.13 & 3.92 & 0.31 & 3.42 & 0.4 & -0.05 & 0.85 & 0.32 & 1.66 \\
Case 5A & 7.89 & 6.75 & 4.15 & 9.02 & 5.27 & 0.33 & 3.15 & -0.12 & 1.33 & 12.32 & 13.15 & 9.45 \\
\hline
\end{tabular}

Table B.2. CPS2 Performance

\begin{tabular}{ccccccccccccc}
\hline Month & \multicolumn{1}{c}{ Jan } & \multicolumn{1}{c}{ Feb } & \multicolumn{1}{c}{ Mar } & \multicolumn{1}{c}{ Apr } & \multicolumn{1}{c}{ May } & \multicolumn{1}{c}{ Jun } & \multicolumn{1}{c}{ Jul } & \multicolumn{1}{c}{ Aug } & Sep & Oct & Nov & Dec \\
\hline Case 1A & 97.269 & 99.702 & 99.954 & 99.699 & 92.894 & 97.222 & 90.532 & 96.829 & 98.217 & 98.032 & 99.768 & 99.861 \\
Case 2A & 97.292 & 99.826 & 99.954 & 99.884 & 93.426 & 97.245 & 93.657 & 96.574 & 98.727 & 98.981 & 99.745 & 99.792 \\
Case 3A & 96.991 & 99.702 & 99.931 & 99.699 & 93.31 & 97.129 & 93.009 & 96.25 & 98.402 & 98.287 & 99.467 & 99.907 \\
Case 4A & 95.81 & 99.777 & 99.977 & 99.745 & 94.213 & 97.245 & 91.875 & 97.685 & 98.889 & 97.199 & 98.495 & 99.259 \\
Case 5A & 90.625 & 93.848 & 96.296 & 92.637 & 93.75 & 97.499 & 93.079 & 96.644 & 97.777 & 88.218 & 89.048 & 89.072 \\
\hline
\end{tabular}

Table B.3. Duration (hour) of Regulation Up Reserve Shortage

\begin{tabular}{ccccccccccccc}
\hline Month & Jan & Feb & Mar & Apr & May & Jun & Jul & Aug & Sep & Oct & Nov & Dec \\
\hline Case 1A & 11 & 1 & 1 & 4 & 61 & 21 & 77 & 30 & 10 & 28 & 5 & 1 \\
Case 2A & 10 & 3 & 1 & 3 & 60 & 20 & 51 & 36 & 7 & 26 & 9 & 1 \\
Case 3A & 12 & 3 & 1 & 4 & 58 & 23 & 69 & 35 & 7 & 18 & 8 & 1 \\
Case 4A & 16 & 2 & 0 & 2 & 56 & 21 & 77 & 22 & 6 & 17 & 15 & 2 \\
Case 5A & 32 & 7 & 2 & 2 & 45 & 16 & 59 & 21 & 8 & 23 & 16 & 4 \\
\hline
\end{tabular}

Table B.4. Duration (hour) of Regulation Down Reserve Shortage

\begin{tabular}{ccccccccccccc}
\hline Month & Jan & Feb & Mar & Apr & May & Jun & Jul & Aug & Sep & Oct & Nov & Dec \\
\hline Case 1A & 22 & 0 & 0 & 0 & 54 & 12 & 49 & 6 & 7 & 22 & 1 & 0 \\
Case 2A & 24 & 0 & 0 & 0 & 52 & 12 & 28 & 5 & 5 & 22 & 4 & 0 \\
Case 3A & 23 & 0 & 0 & 0 & 53 & 11 & 40 & 6 & 6 & 26 & 4 & 0 \\
Case 4A & 24 & 0 & 0 & 13 & 56 & 12 & 43 & 1 & 5 & 32 & 9 & 5 \\
Case 5A & 84 & 71 & 54 & 85 & 57 & 10 & 34 & 3 & 16 & 111 & 112 & 102 \\
\hline
\end{tabular}


Table B.5. Runtime (generator-hour) Statistics of Peaking Units Dispatched by Operator Model

\begin{tabular}{ccccccccccccc}
\hline Month & Jan & Feb & Mar & Apr & May & Jun & Jul & Aug & Sep & Oct & Nov & Dec \\
\hline Case 1A & 173 & 10 & 17 & 21 & 264 & 331 & 1001 & 507 & 212 & 51 & 101 & 67 \\
Case 2A & 156 & 23 & 14 & 21 & 266 & 313 & 1002 & 471 & 223 & 68 & 55 & 75 \\
Case 3A & 139 & 24 & 14 & 19 & 262 & 311 & 981 & 451 & 213 & 66 & 66 & 100 \\
Case 4A & 149 & 21 & 13 & 13 & 224 & 287 & 582 & 450 & 200 & 76 & 87 & 131 \\
Case 5A & 216 & 18 & 17 & 9 & 210 & 261 & 536 & 418 & 161 & 264 & 54 & 173 \\
\hline
\end{tabular}

Table B.6. Operator Manual Dispatch (generator-hour) on Non-Regulation Units

\begin{tabular}{ccccccccccccc}
\hline Month & Jan & Feb & Mar & Apr & May & Jun & Jul & Aug & Sep & Oct & Nov & Dec \\
\hline Case 1A & 501 & 357 & 664 & 595 & 401 & 542 & 813 & 677 & 498 & 525 & 590 & 5 \\
Case 2A & 614 & 355 & 640 & 734 & 398 & 522 & 810 & 665 & 494 & 562 & 609 & 5 \\
Case 3A & 618 & 355 & 649 & 772 & 479 & 521 & 794 & 652 & 435 & 561 & 592 & 41 \\
Case 4A & 636 & 549 & 675 & 697 & 398 & 497 & 818 & 615 & 488 & 530 & 627 & 79 \\
Case 5A & 744 & 638 & 714 & 504 & 440 & 495 & 715 & 592 & 391 & 590 & 669 & 138 \\
\hline
\end{tabular}





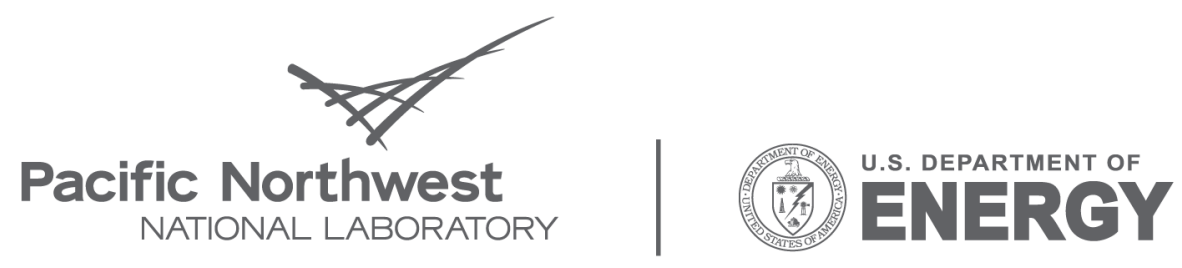

Proudly Operated by Battelle Since 1965

902 Battelle Boulevard

P.O. Box 999

Richland, WA 99352

1-888-375-PNNL (7665)

www.pnl.gov 